In cooperation with the Texas Commission on Environmental Quality

\title{
Water-Quality and Ancillary Data Collected From the Arroyo Colorado Near Rio Hondo, Texas, 2006
}

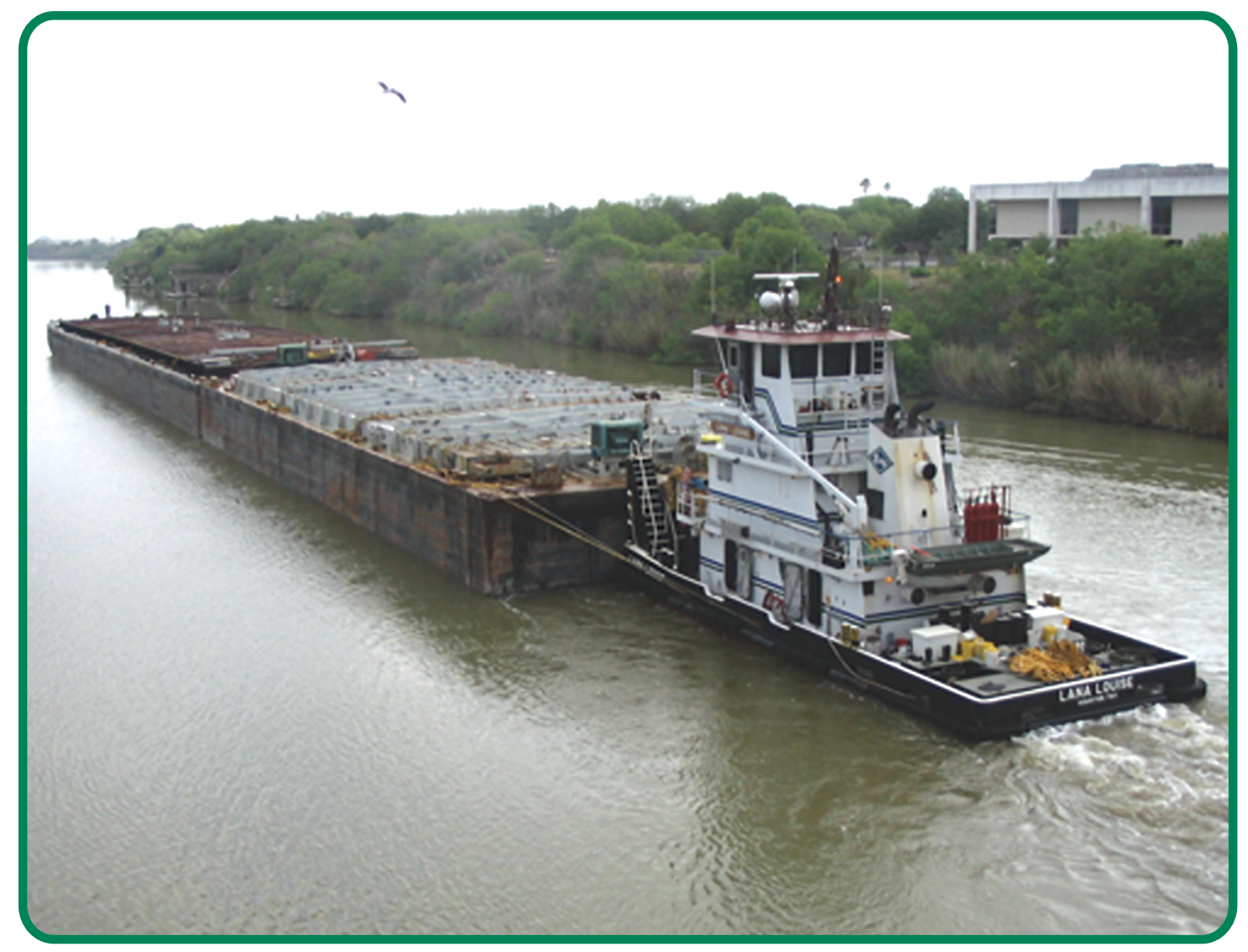

Data Series 255 
Cover. Arroyo Colorado downstream from Rio Hondo lift bridge, June 2005 (photograph courtesy of Roger Miranda, Texas Commission on Environmental Quality). 


\section{Water-Quality and Ancillary Data Collected From the Arroyo Colorado Near Rio Hondo, Texas, 2006}

By Meghan C. Roussel, Michael G. Canova, William H. Asquith, and

Richard L. Kiesling

In cooperation with the Texas Commission on Environmental Quality

Data Series 255

U.S. Department of the Interior

U.S. Geological Survey 


\title{
U.S. Department of the Interior DIRK KEMPTHORNE, Secretary
}

\author{
U.S. Geological Survey \\ Mark D. Myers, Director
}

\section{U.S. Geological Survey, Reston, Virginia: 2007}

For more information about the USGS and its products:

Telephone: 1-888-ASK-USGS

World Wide Web: http://www.usgs.gov/

Any use of trade, product, or firm names in this publication is for descriptive purposes only and does not imply endorsement by the U.S. Government.

Although this report is in the public domain, permission must be secured from the individual copyright owners to reproduce any copyrighted materials contained within this report.

Suggested citation:

Roussel, M.C., Canova, M.G., Asquith, W.H., and Kiesling, R.L., 2007, Water-quality and ancillary data collected from the Arroyo Colorado near Rio Hondo, Texas, 2006: U.S. Geological Survey Data Series 255, $46 \mathrm{p}$. 


\section{Contents}

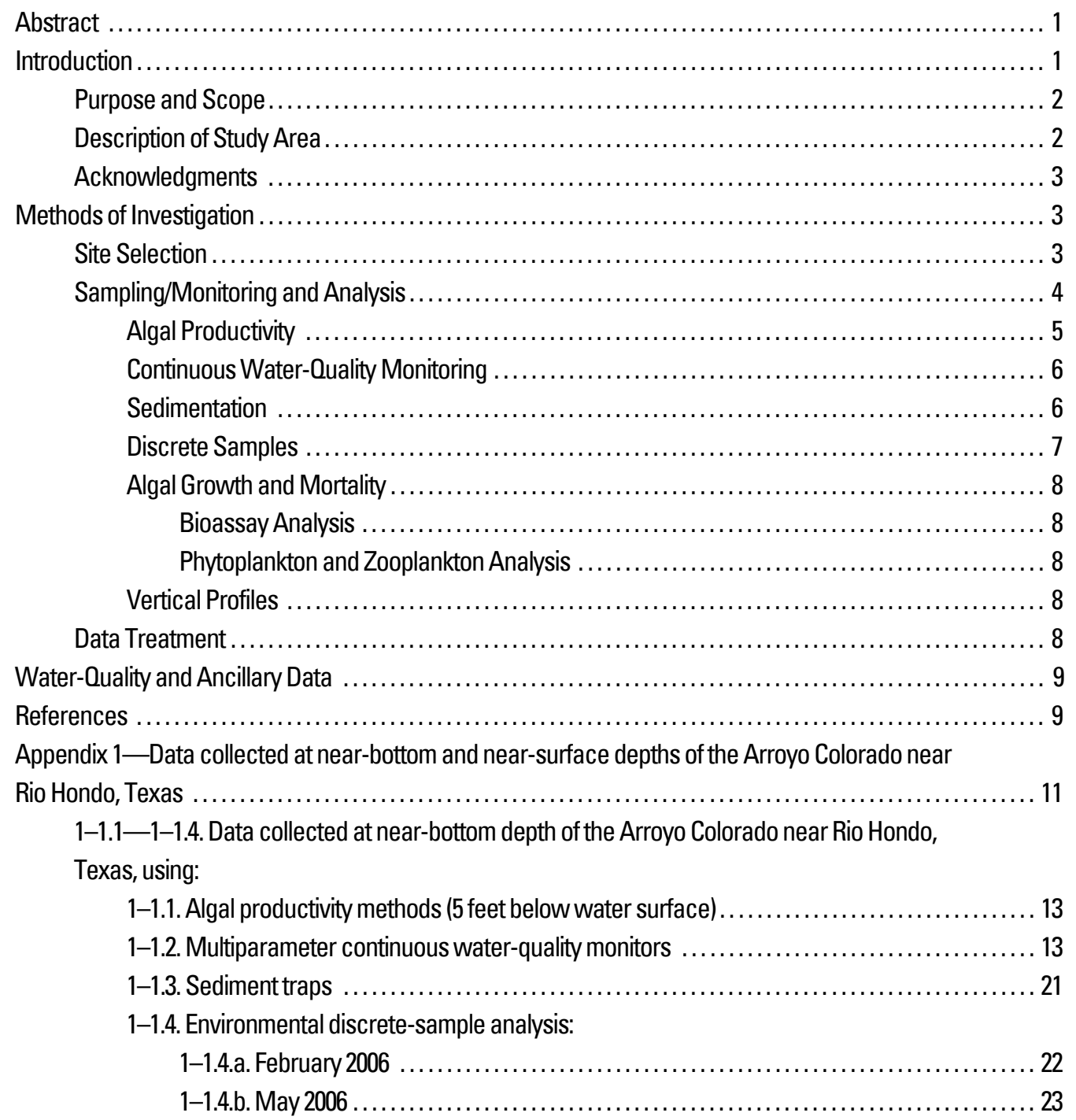

1-2.1 -1-2.4. Data collected at near-surface depth of the Arroyo Colorado near Rio Hondo,

Texas, using:

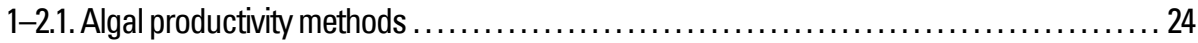

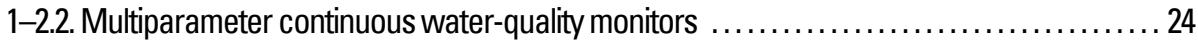

1-2.3. Algal growth and mortality analyses:

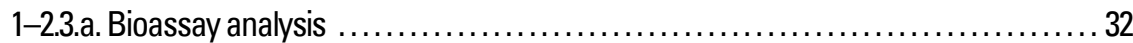

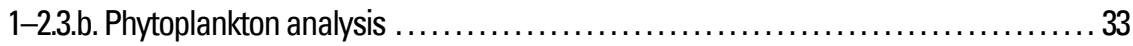

$1-2.3 . c$. Zooplankton analysis ........................................... 35

1-2.4. Environmental discrete-sample analysis:

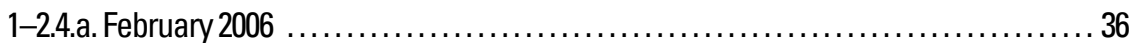



1-3. Data collected for quality assurance/quality control from the Arroyo Colorado near Rio Hondo, Texas 
Appendix 2 -Vertical profile data collected from the Arroyo Colorado near Rio Hondo, Texas ............. 39

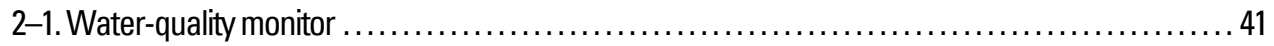



\section{Figures}

1-2. Maps showing:

1. Arroyo Colorado Basin, Texas

2. Water-quality data-collection sites on the tidal segment (2201) of the Arroyo Colorado,

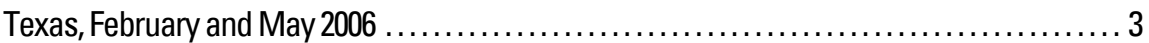

3. Schematic diagram of water-quality data collection in the tidal segment (2201) of the Arroyo

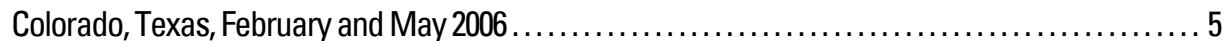

4-7. Photographs showing:

4. Light-dark bottle experiment being deployed in the Arroyo Colorado near Rio Hondo,

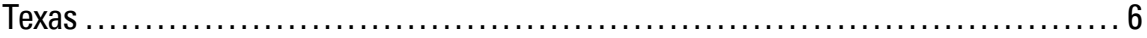

5. Continuous multiparameter water-quality monitor configuration and deployment in the Arroyo Colorado near Rio Hondo, Texas ................................ 7

6. Sediment trap deployment in the Arroyo Colorado near Rio Hondo, Texas ............... 7

7. Near-bottom water being collected using the Kemmerer sampler in the Arroyo Colorado near Rio Hondo, Texas

\section{Tables}

1. Water-quality data-collection sites on the tidal segment (2201) of the Arroyo Colorado,



2. Physical characteristics with measurement methods specified by the Texas Commission

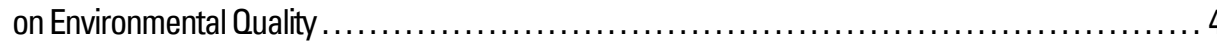

3. Analytes with measurement methods specified by Texas Commission on Environmental



\section{Datum}

Horizontal coordinate information is referenced to North American Datum of 1983 (NAD 83). 


\title{
Water-Quality and Ancillary Data Collected From the Arroyo Colorado Near Rio Hondo, Texas, 2006
}

\author{
By Meghan C. Roussel, Michael G. Canova, William H. Asquith, and Richard L. Kiesling
}

\section{Abstract}

The Arroyo Colorado is in the lower Rio Grande Valley of southern Texas and extends from near Mission, Texas, eastward to the Laguna Madre estuarine and coastal marine system, which separates Padre Island from the Texas mainland. Streamflow in the Arroyo Colorado primarily is sustained by effluent from municipal wastewater-treatment plants along the stream banks. Since 1986, the tidal segment of the Arroyo Colorado from the port of Harlingen to the Laguna Madre has been designated by the State of Texas as an impaired water body because of low dissolved oxygen concentrations. Efforts to develop predictive water-quality models for the tidal segment of the Arroyo Colorado have been hampered by a lack of physical, biological, and biochemical data. Specifically, data on primary algal productivity, nutrient cycling, sediment deposition rates, and the relations between these processes and dissolved oxygen dynamics in the stream have been inadequate to support water-quality modeling efforts. The U.S. Geological Survey, in cooperation with the Texas Commission on Environmental Quality, did a study in 2006 to collect data associated with primary algal productivity, nutrient cycling, and dissolved oxygen dynamics in the tidal segment (2201) of the Arroyo Colorado near Rio Hondo. Specific objectives of the study were to (1) characterize water quality by measuring basic properties; (2) characterize the concentrations of carbon and nutrients, biochemical oxygen demand, total organic carbon, total suspended solids, and volatile suspended solids; (3) measure the seasonal differences of nutrient-dependent algal growth and algal production in the water column; (4) measure oxygen respiration or production rates; and (5) measure rates of sediment deposition.

\section{Introduction}

The Arroyo Colorado is in the lower Rio Grande Valley of southern Texas and extends from near Mission, Tex., eastward to the Laguna Madre estuarine and coastal marine system, which separates South Padre Island from the Texas mainland (fig. 1). Streamflow in the Arroyo Colorado primarily is sustained by effluent from municipal wastewater-treatment plants along the stream banks. Additional streamflow is contributed by irrigation return flow, storm runoff, and other sources. The Arroyo Colorado is used as a floodway; an inland waterway; and a recreational area for boating, fishing, and swimming; and is an important nursery and foraging area for crab, shrimp, and several types of marine fish (Raines and Miranda, 2002).

The Texas Commission on Environmental Quality (TCEQ) has classified two distinct stream sections (fig. 1) of the Arroyo Colorado (Texas Natural Resource Conservation Commission, 1997) on the basis of the physical characteristics of the stream. Segment 2201 extends downstream from the port of Harlingen to the Laguna Madre at the border of Cameron and Willacy Counties, is tidally influenced, provides habitat to a diverse range of aquatic organisms, and has contactrecreational and high aquatic life designated uses. Segment 2202 extends upstream from the port of Harlingen to Perezville in Hidalgo County, is nontidal, is the shallower and narrower of the two segments, and also has contact-recreational and intermediate aquatic life designated uses. Segment 2202 is not considered further in this report.

Since 1986, the tidal segment of the Arroyo Colorado has been designated by the State of Texas as an impaired water body because of low dissolved oxygen (DO) concentrations (R.M. Miranda, Texas Commission on Environmental Quality, oral commun., 2005). Efforts to develop predictive waterquality models for the Arroyo Colorado have been hampered by a lack of physical, biological, and biochemical data. These data are necessary to provide information on primary algal productivity, nutrient cycling, sediment deposition rates, and the relations between these processes and DO dynamics used to support many types of water-quality models. The catalyst for the investigation described here was the need for more data to increase the reliability of predictive models.

The U.S. Geological Survey (USGS), in cooperation with the TCEQ, did a study in 2006 to collect data associated with primary algal productivity, nutrient cycling and DO dynamics in the tidal segment (2201) of the Arroyo Colorado near Rio Hondo. Specific objectives of the study were to (1) characterize 


\section{Water-Quality and Ancillary Data Collected From the Arroyo Colorado Near Rio Hondo, Texas, 2006}

water quality by measuring 15 -minute values of $\mathrm{DO}, \mathrm{pH}$, specific conductance, and temperature at two depths (near bottom and near surface) for each of four sites in the tidal segment over a 24-hour or longer period during one data-collection period in February 2006 and one in May 2006; (2) characterize the concentrations of carbon and nutrients (including pertinent nitrogen and phosphorus species), biochemical oxygen demand (BOD), total organic carbon (TOC), total suspended solids (TSS), and volatile suspended solids (VSS) during the two periods; (3) measure the seasonal (as provided by data from the two collection periods) differences of nutrient-dependent algal growth and algal productivity in the water column; (4) measure oxygen respiration or production rates; and (5) measure rates of sediment deposition during each of the two data-collection periods.

\section{Purpose and Scope}

The purpose of this report is to describe the methods for data sampling and analysis and to present the water-quality and ancillary data collected in the tidal segment (2201) of the Arroyo Colorado near Rio Hondo, Tex., during two datacollection periods, one in February and one in May 2006.
Site selection, sampling/monitoring and analysis, and postcollection data treatment, including quality assurance/quality control (QA/QC), are described. The data (in tabular format) are presented in two appendixes, one containing the data collected at near-bottom and near-surface depths and one containing the data collected along vertical profiles.

\section{Description of Study Area}

The study area is in southern Texas in the lower Rio Grande Valley (fig. 1). The Arroyo Colorado flows about 90 miles from west to east and has a contributing drainage area (basin) of about 700 square miles. The study area is in the neotropical Southern Coastal Plain physiographic province (National Oceanic and Atmospheric Administration, 1996) and is characterized by long, hot summers and short, mild winters. The mean annual temperature is about 73 degrees Fahrenheit $\left({ }^{\circ} \mathrm{F}\right)$, and mean monthly temperatures range from $58{ }^{\circ} \mathrm{F}$ (January) to $84^{\circ} \mathrm{F}$ (July). Mean annual evaporation in the study area is about 58 inches. Mean annual rainfall is about 21 inches at the western limit and 27 inches at the eastern limit of the study area.

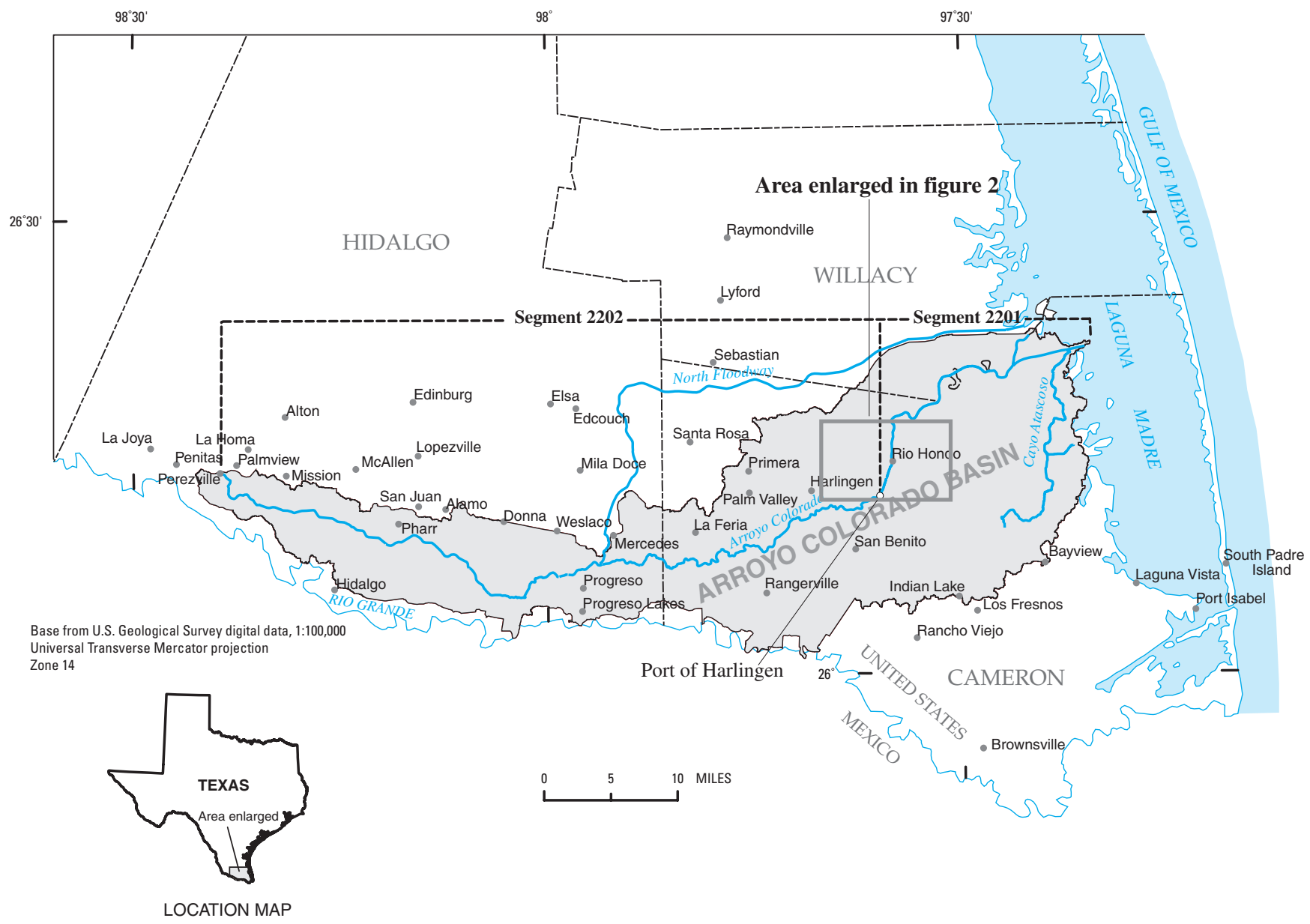

Figure 1. Arroyo Colorado Basin, Texas (modified from Raines and Miranda, 2002, fig. 1). 


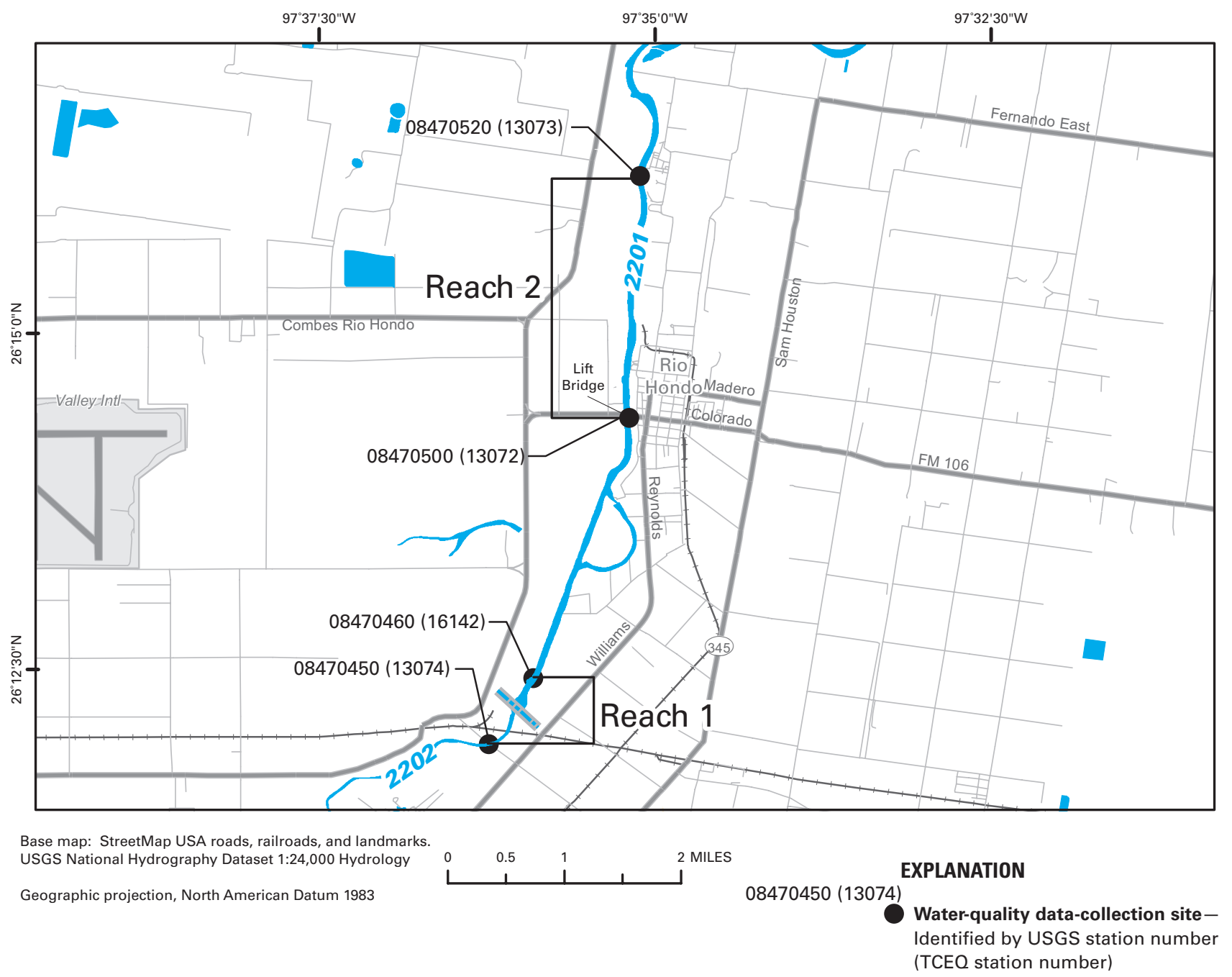

Figure 2. Water-quality data-collection sites on the tidal segment (2201) of the Arroyo Colorado, Texas, February and May 2006.

The lower Rio Grande Valley has flat terrain and is extensively cultivated and irrigated for agriculture. Water for irrigation is transported from the Rio Grande through canals to the fields by operators of numerous irrigation districts. The irrigation districts provide water for irrigation of citrus, sugar cane, and several row crops that include corn, grain sorghum, and cotton. Irrigation practices generally consist of periodic field flooding during periods of insufficient rainfall to produce economically viable crop yields. Urban development is extensive in many areas adjacent to the Arroyo Colorado, particularly in the western and central parts of the drainage area.

There are 21 permitted dischargers in the Arroyo Colorado Basin: 16 are municipal wastewater-treatment plants, three are industrial, and two are shrimp farm operations. The limits of the municipal discharge permits range from 0.4 to 10 million gallons per day (Raines and Miranda, 2002).

\section{Acknowledgments}

The authors recognize the contributions of Roger Miranda, TCEQ Total Maximum Daily Load (TMDL) Project Manager, and Faith Hambleton, TCEQ TMDL Program Manager.

\section{Methods of Investigation}

\section{Site Selection}

Two reaches, each of which is bounded by two sampling sites within segment 2201, were specified by TCEQ as areas of particular interest (fig. 2; table 1). Site selection was based on the requirements of the TCEQ TMDL program (R.M. Miranda, Texas Commission on Environmental Quality, oral commun., 
2005). The first reach is between USGS stations 08470450 and 08470460, near the boundary between the tidal and nontidal segments of the Arroyo Colorado, and the second reach is between USGS stations 08470500 and 08470520 , downstream of the lift bridge on FM 106 in Rio Hondo, where summer fish kills are common (R.M. Miranda, Texas Commission on Environmental Quality, oral commun., 2005). The sites provide potential calibration points for modeling and other assessments.

Table 1. Water-quality data-collection sites on the tidal segment (2201) of the Arroyo Colorado, Texas, February and May 2006.

[USGS, U.S. Geological Survey; TCEQ, Texas Commission on Environmental Quality]

\begin{tabular}{ccccc}
\hline $\begin{array}{c}\text { USGS } \\
\text { station } \\
\text { no. }\end{array}$ & $\begin{array}{c}\text { TCE0 } \\
\text { station } \\
\text { no. }\end{array}$ & Station name & $\begin{array}{c}\text { Latitude } \\
\text { (decimal } \\
\text { degrees) }\end{array}$ & $\begin{array}{c}\text { Longitude } \\
\text { (decimal } \\
\text { degrees) }\end{array}$ \\
\hline 08470450 & 13074 & $\begin{array}{c}\text { Arroyo Colorado above } \\
\text { Rio Hondo, Texas }\end{array}$ & 26.195 & 97.602 \\
08470460 & 16142 & $\begin{array}{c}\text { Arroyo Colorado at the } \\
\text { port of Harlingen, Rio } \\
\text { Hondo, Texas }\end{array}$ & 26.203 & 97.596 \\
08470500 & 13072 & $\begin{array}{c}\text { Arroyo Colorado at FM } \\
\text { 106 at Rio Hondo, } \\
\text { Texas }\end{array}$ & 26.235 & 97.584 \\
08470520 & 13073 & $\begin{array}{c}\text { Arroyo Colorado at } \\
\text { Camp Perry near Rio } \\
\text { Hondo, Texas }\end{array}$ & 26.265 & 97.583 \\
\hline
\end{tabular}

\section{Sampling/Monitoring and Analysis}

Because of the tidal nature of the segment, two depths (near bottom and near surface) at each of the four selected sites were designated for sampling. A schematic diagram of waterquality sampling for each data-collection period, one in February 2006 and one in May 2006, is shown in figure 3. For nearbottom depths at each site, algal productivity measurements were taken (using light-dark bottle experiments), multiparameter water-quality monitors were deployed, sediment traps (to measure sedimentation rates) were deployed, and discrete environmental samples were collected. For near-surface depths at each site, algal productivity measurements were taken, multiparameter water-quality monitors were deployed, water samples for bioassay analysis were collected, water samples for phytoplankton and zooplankton analysis were collected, and discrete environmental samples were collected. Also at each site, vertical profiles of water-quality characteristics and photosynthetically active radiation (PAR) were conducted as well as a variety of field measurements. QA/QC samples were collected at selected sites. Physical characteristics (from field measurements) and respective measurement methods specified by TCEQ are listed in table 2, and analytes (from environmental samples) and respective measurement methods specified by TCEQ are listed in table 3. All other data collected were analyzed using standard USGS methods.

Table 2. Physical characteristics with measurement methods specified by Texas Commission on Environmental Quality.

[TCEQ SOP, Texas Commission on Environmental Quality (2003) Standard Operating Procedure; EPA, U.S. Environmental Protection Agency (1983); mg/L, milligrams per liter; $\mu \mathrm{S} / \mathrm{cm}$, microsiemens per centimeter at 25 degrees Celsius; ${ }^{\circ} \mathrm{C}$, degrees Celsius; $\mu \mathrm{E} / \mathrm{m}^{2} / \mathrm{sec}$, microEinsteins per square meter per second; ppt, parts per thousand; SM, Standard Method (American Public Health Association, 1998); ft/sec, feet per second; FNMU, formazin nephelometric units]

\begin{tabular}{lll}
\hline \multicolumn{1}{c}{ Physical characteristic } & \multicolumn{1}{c}{ Units } & \multicolumn{1}{c}{ Method } \\
\hline Depth & feet & TCEQ SOP \\
$\mathrm{pH}$ & $\mathrm{pH}$ units & EPA 150.1 and TCEQ SOP \\
Dissolved oxygen & $\mathrm{mg} / \mathrm{L}$ & EPA 150.1 and TCEQ SOP \\
Specific conductance & $\mu \mathrm{S} / \mathrm{cm}$ & EPA 150.1 and TCEQ SOP \\
Temperature & ${ }^{\circ} \mathrm{C}$ & EPA 150.1 and TCEQ SOP \\
Secchi depth & $\mathrm{feet}$ & TCEQ SOP \\
Light & $\mu \mathrm{E} / \mathrm{m}^{2} / \mathrm{sec}$ & Wetzel and Likens (1979) \\
Days since significant rainfall & $\mathrm{days}$ & TCEQ SOP \\
Salinity & $\mathrm{ppt}$ & SM 2520 and TCEQ SOP \\
Velocity & $\mathrm{ft} / \mathrm{sec}$ & TCEQ SOP \\
Air temperature & ${ }^{\circ} \mathrm{C}$ & TCEQ SOP \\
Turbidity & $\mathrm{FNMU}$ & EPA 160.1 \\
\hline
\end{tabular}




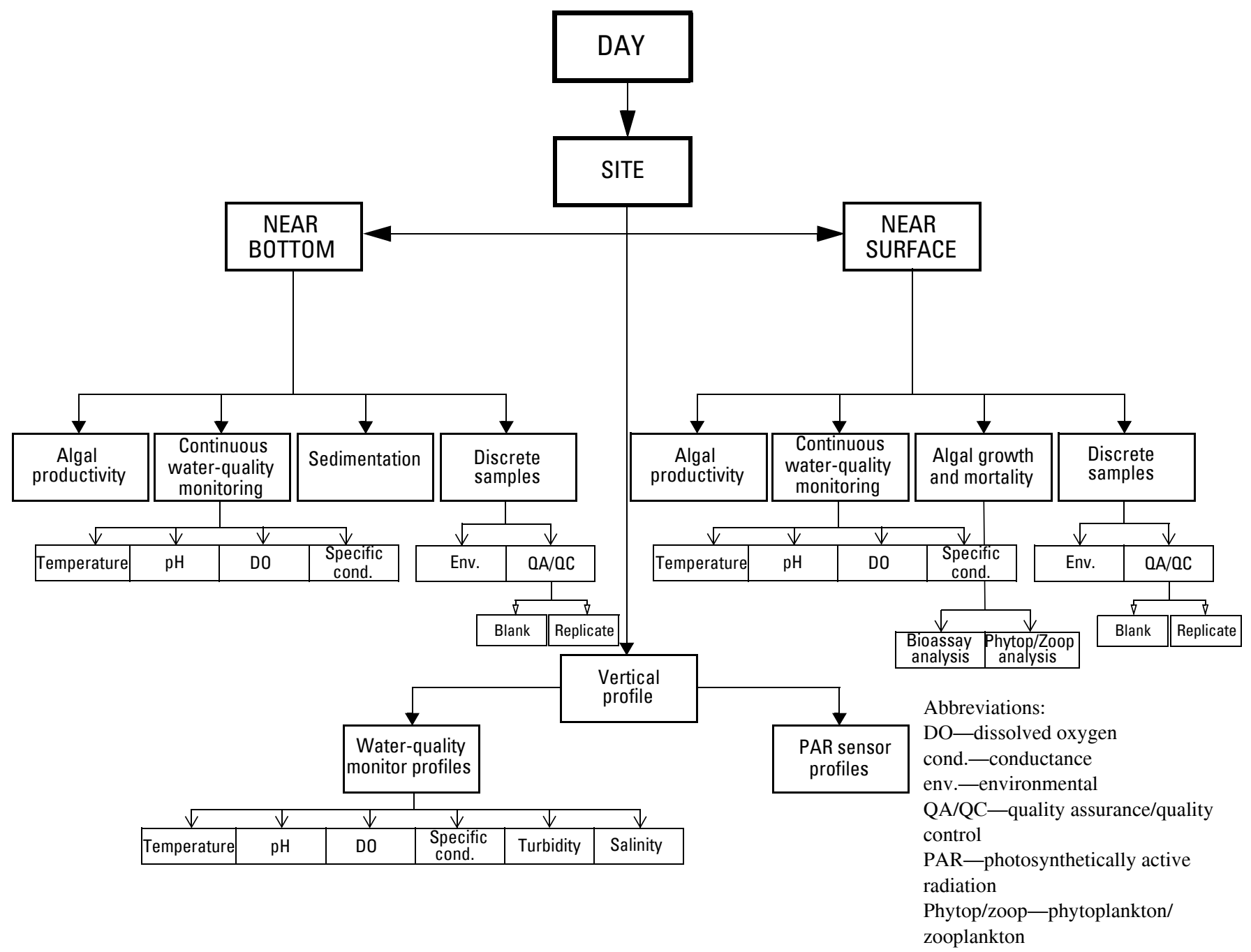

Figure 3. Schematic diagram of water-quality data collection in the tidal segment of the Arroyo Colorado, Texas, February and May 2006.

Water samples collected at the four sites during each of the two data-collection periods were analyzed for a suite of waterquality constituents that included 5-day BOD, chloride and sulfate, chlorophyll $a$ and pheophytin $a$, nutrients, particulate carbon and particulate nitrogen, and TSS and VSS. In addition, bioassay analyses, sediment flux measurements, and standing light-dark bottle productivity measurements were made. Field measurements consisted of air temperature, barometric pressure, Secchi depth, wind speed, and vertical profile data that comprised DO, PAR, pH, salinity, specific conductance, turbidity, and water temperature. The scope of work did not include measurements of streamflow.

\section{Algal Productivity}

Algal productivity measurements, which describe watercolumn primary productivity and are determined by changes in DO concentrations using light-dark bottle methods, were taken at one site per reach (two sites total) at near-surface (about 1 foot below the water surface) and near-bottom (about 5 feet below the water surface) depths. DO and temperature profiles were measured in-situ at each site. These measurements were used as initial conditions with which to compare the final values obtained from the light-dark bottle experiments. A replicate set (two each) of clear and dark plastic-coated bottles were filled with water collected at the two specified depths and sealed with standard ground-glass stoppers to prevent gas exchange with the surrounding water column. Care was taken to shade the bottles during the filling process. Once all the bottles were filled, they were secured to a bottle rack (as shown in fig. 4) and suspended at the depth from which the water was collected. After a minimum of 4 hours of deployment, the light-dark bottles were removed from the water column, and DO and temperature measurements were made. Reaeration was kept to a minimum during final DO and temperature measurements. Start and end times for the instream incubations were recorded, as were DO and temperature measurements. 


\section{Water-Quality and Ancillary Data Collected From the Arroyo Colorado Near Rio Hondo, Texas, 2006}

Table 3. Analytes with measurement methods specified by Texas Commission on Environmental Quality.

[mg/L, milligrams per liter; EPA, U.S. Environmental Protection Agency (1983); N, nitrogen; P, phosphorus; $\mu \mathrm{g} / \mathrm{L}$, micrograms per liter; SM, Standard Method (American Public Health Association, 1998); cells/mL, cells per milliliter; ind./mL, individuals per milliliter]

\begin{tabular}{|c|c|c|}
\hline Analyte & Units & Method \\
\hline Total suspended solids & $\mathrm{mg} / \mathrm{L}$ & EPA 160.2 \\
\hline Total organic carbon & $\mathrm{mg} / \mathrm{L}$ & EPA 415.1 \\
\hline $\begin{array}{l}\text { Dissolved organic } \\
\text { carbon }\end{array}$ & $\mathrm{mg} / \mathrm{L}$ & EPA 415.2 \\
\hline Total dissolved solids & $\mathrm{mg} / \mathrm{L}$ & EPA 160.1 \\
\hline Sulfate & $\mathrm{mg} / \mathrm{L}$ & EPA 300.0 \\
\hline Chloride & $\mathrm{mg} / \mathrm{L}$ & EPA 300.0 \\
\hline $\begin{array}{l}\text { Ammonia-nitrogen, } \\
\text { total }\end{array}$ & $\mathrm{mg} / \mathrm{L}$ as $\mathrm{N}$ & EPA 350.1 \\
\hline $\begin{array}{l}\text { Orthophosphate, } \\
\text { dissolved }\end{array}$ & $\mathrm{mg} / \mathrm{L}$ as $\mathrm{P}$ & EPA 365.1 \\
\hline Total reactive phosphate & $\mathrm{mg} / \mathrm{L}$ & EPA 365.1 \\
\hline $\begin{array}{l}\text { Total phosphorus wet } \\
\text { method }\end{array}$ & $\mathrm{mg} / \mathrm{L}$ as $\mathrm{P}$ & EPA 365.1 \\
\hline $\begin{array}{l}\text { Nitrate/nitrite-nitrogen, } \\
\text { total }\end{array}$ & $\mathrm{mg} / \mathrm{L}$ as $\mathrm{N}$ & EPA 353.2 \\
\hline Total kjeldahl nitrogen & $\mathrm{mg} / \mathrm{L}$ as $\mathrm{N}$ & EPA 351.2 \\
\hline Total nitrogen & $\mathrm{mg} / \mathrm{L}$ as $\mathrm{N}$ & Computation \\
\hline Chlorophyll $a$ & $\mu \mathrm{g} / \mathrm{L}$ & SM $10200 \mathrm{H}$ \\
\hline Pheophytin $a$ & $\mu \mathrm{g} / \mathrm{L}$ & SM $10200 \mathrm{H}$ \\
\hline Phytoplankton & cells $/ \mathrm{mL}$ & SM $10200 \mathrm{~F}$ \\
\hline Zooplankton & ind./mL & SM $10200 \mathrm{G}$ \\
\hline Particulate carbon & $\mathrm{mg} / \mathrm{L}$ & EPA 440.0 \\
\hline Particulate nitrogen & $\mathrm{mg} / \mathrm{L}$ & EPA 440.0 \\
\hline $\begin{array}{l}\text { 5-day biochemical } \\
\text { oxygen demand }\end{array}$ & $\mathrm{mg} / \mathrm{L}$ & SM 5210 \\
\hline $\begin{array}{l}\text { Volatile suspended } \\
\text { solids }\end{array}$ & $\mathrm{mg} / \mathrm{L}$ & EPA 160.4 \\
\hline Algal productivity & $\mathrm{mg} / \mathrm{L}$ & SM 10300D2 \\
\hline Algal growth & day $^{-1}$ & Lehman and Sandgren (1985) \\
\hline Algal mortality & day $^{-1}$ & Lehman and Sandgren (1985) \\
\hline
\end{tabular}

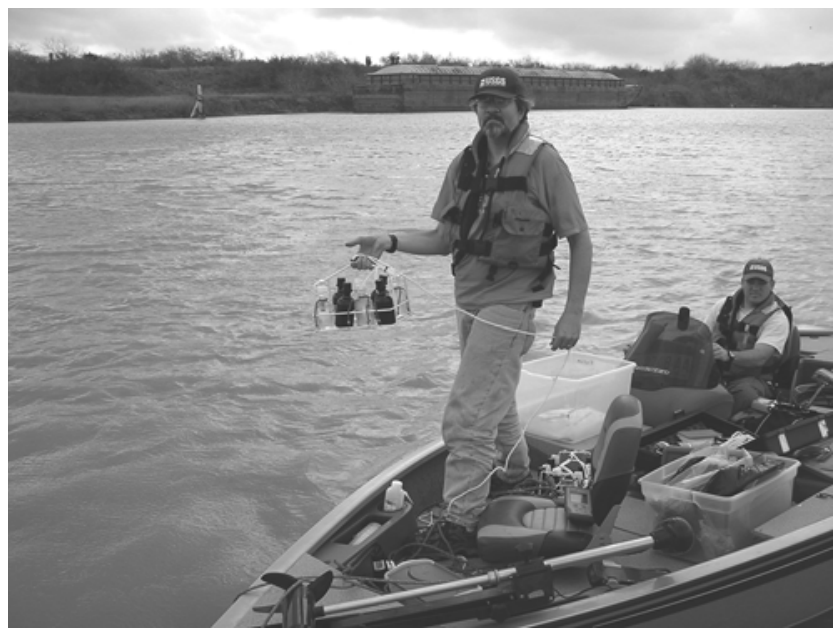

Figure 4. Light-dark bottle experiment being deployed in the Arroyo Colorado near Rio Hondo, Texas.
Standard light-dark bottle computational methods were used to determine gross primary productivity (GPP) and net primary productivity (NPP) or gross respiration and net respiration, where respiration is signified by a negative value, for each site at each of the two depths (American Public Health Association, 1998). GPP is computed by subtracting the final DO concentration in the dark bottle (DB) from the final DO concentration in the light bottle (LB) and then dividing by the incubation period ( $\Delta \mathrm{t}$ ) and a conversion factor to convert liters to cubic meters (milligrams of oxygen/cubic meter/day). The equation for GPP is as follows:

$$
\mathrm{GPP}=\frac{(\mathrm{LB}-\mathrm{DB})}{\Delta \mathrm{t}} \times 1000 .
$$

NPP (summation of all metabolic processes) is computed by subtracting the initial light bottle DO concentration (ILB) from the final DO concentration in the light bottle (LB) and then dividing by the incubation period $(\Delta \mathrm{t})$ and a conversion factor to convert liters to cubic meters. The equation for NPP is as follows:

$$
\mathrm{NPP}=\frac{(\mathrm{LB}-\mathrm{ILB})}{\Delta \mathrm{t}} \times 1000
$$

In this method, changes in the mass of oxygen in the closed bottles during the incubation period are used to estimate rates of primary productivity. In this study, one replicate in addition to the environmental sample at each depth allowed for an averaging of the computed rates. Procedures follow those outlined in method 10300D2 of "Standard Methods for the Examination of Water and Wastewater" (American Public Health Association, 1998).

\section{Continuous Water-Quality Monitoring}

Multiparameter water-quality monitors were deployed at the near-surface and near-bottom depths at all four sites to collect 15-minute $\mathrm{DO}, \mathrm{pH}$, specific conductance, and temperature data. The instruments were deployed for a minimum of 24 hours during concurrent collection of other data. The monitor configuration and deployment procedure are shown in figure 5. Procedures for 24-hour continuous multiparameter field data collection follow standard protocols (Texas Commission on Environmental Quality, 2003; U.S. Geological Survey, 2006).

\section{Sedimentation}

Sedimentation is the vertical deposition of mass per unit time per unit area (mass flux) in the water column. Sedimentation is of critical importance to estimates of sediment movement and algal mortality within the context of water-quality models. Sedimentation represents the transport of particulate chemical species vertically through the water column with the explicit assumption that the chemical species reach the sediments. Sediment traps are used to measure the deposition of particulates over a specified period of time by integrating particle deposition 

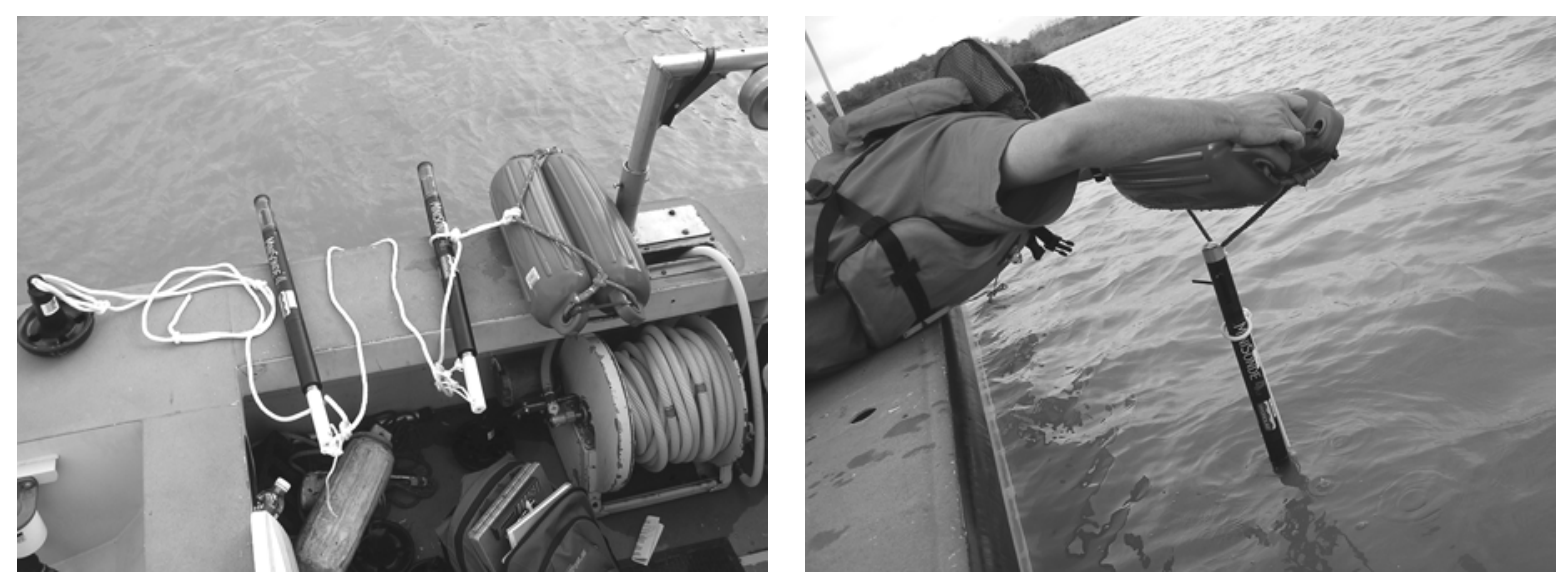

Figure 5. Continuous multiparameter water-quality monitor configuration and deployment in the Arroyo Colorado near Rio Hondo, Texas.

through time in a collection cylinder. The traps are cylindrical collectors closed at the bottom and open at the top that catch mineral particles, plant debris, refractory organic material, dead phytoplankton and zooplankton, the feces of zooplankton and fish, and other detritus. The traps are attached to a line with floats on the surface and a weight at the bottom to keep the trap vertical.

At each site, 4-inch-diameter traps were deployed in pairs (fig. 6). After a specified period of time, approximately 48 hours for this study, the traps were recovered. The exact time of deployment and retrieval were noted for each trap to allow for the computation of a flux. For retrieval, traps were pulled to the surface, ensuring the cylindrical collectors remained vertical; the cylinders then were emptied into 9-liter carboys. The collected material was weighed and analyzed for TSS and VSS and, during the February data-collection period, particulate carbon and nitrogen. The mass of collected material divided by the collection area and the deployment time of the traps yields the sedimentation flux. Sediment traps were deployed during each of the two data-collection periods at all four sites.

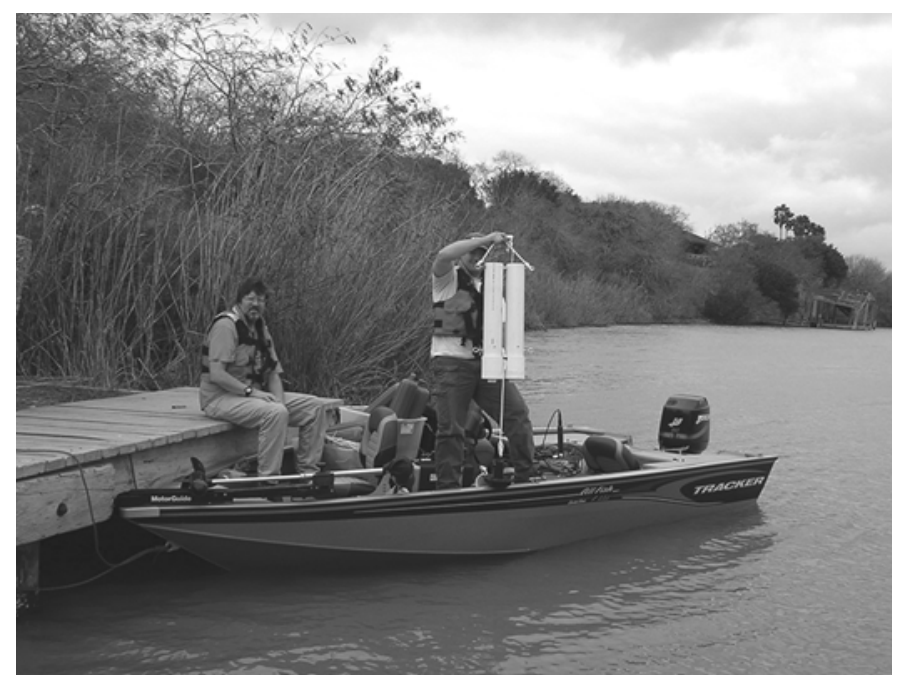

Figure 6. Sediment trap deployment in the Arroyo Colorado near Rio Hondo, Texas.

\section{Discrete Samples}

The USGS collected water samples in the tidal segment of the Arroyo Colorado during each of the two data-collection periods to identify seasonal differences in water quality. Samples were collected on 2 consecutive days at near-surface and near-bottom depths at the four sites. Near-surface samples were collected by the grab-sample method-direct filling of sample bottles from a boat. Near-bottom water samples were collected using a Kemmerer sampler as shown in figure 7. The Kemmerer sampler consists of a hollow cylinder with remotely activated stoppers at each end activated by a weighted messenger. Samples requiring field filtration were filtered in environmental chambers, made up of clear polyethylene bags supported by plastic frames, to minimize the potential for contamination. Equipment that came into contact with the sample was rinsed three times with deionized water between use at each sampling site. The different sampling methods used for sampling nearsurface (grab sample) and near-bottom (Kemmerer sampler) water prevented contamination between samples at each site.

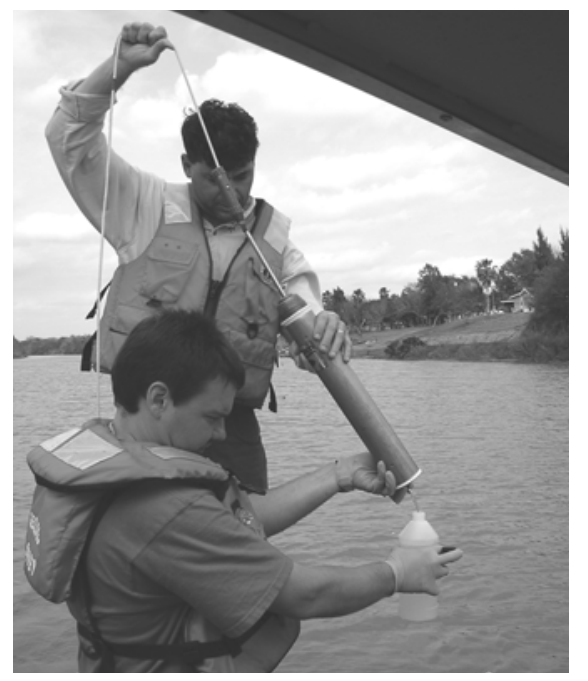

Figure 7. Near-bottom water being collected using the Kemmerer sampler in the Arroyo Colorado near Rio Hondo, Texas. 


\section{Water-Quality and Ancillary Data Collected From the Arroyo Colorado Near Rio Hondo, Texas, 2006}

\section{Algal Growth and Mortality}

\section{Bioassay Analysis}

Algal population growth was determined using standard nutrient-addition bioassays (Lehman and Sandgren, 1985). Bioassay analyses were conducted in the laboratory under ambient temperature and light conditions on water samples collected at each of the four sites at near-surface depths. Water was transported to the laboratory and allocated to one of four treatment groups: control, phosphate $\left(\mathrm{PO}_{4}\right)$ addition, nitrate $\left(\mathrm{NO}_{3}\right)$ addition, or combined $\mathrm{PO}_{4}+\mathrm{NO}_{3}$ addition. Each treatment consisted of four cultures that were monitored for growth every other day for 10 days. Zooplankton were removed from the bioassay water using a 153-micrometer-mesh sieve. Changes in algal biomass were measured using an acclimated growth-rate method where algal populations are measured using in-vivo fluorescence (IVF). An exponential growth model was fit to the IVF data for each culture, providing four estimates of algal population growth rate per treatment per site. Growth rates were used to determine where nutrient additions appreciably enhanced algal growth. Nutrient addition and dilution bioassays were used to simultaneously estimate algal growth rates and grazing mortality rates following the methods of Lehman and Sandgren (1985).

Algal mortality rates were determined using experimental grazer density gradients. Zooplankton were collected from the Arroyo Colorado at each site and were used to increase animal density to ambient levels observed in the field. Micro-grazers also were manipulated through the use of dilution of ambient water using particle-free Arroyo Colorado water. Any observed negative changes in algal growth rates were attributed to the density of grazers in the experimental treatments. Algal mortality rates were assumed to be equal to these negative growth rates.

\section{Phytoplankton and Zooplankton Analysis}

Preserved phytoplankton samples and preserved zooplankton samples were used to estimate ambient population densities at all four sites. Phytoplankton cell counts and zooplankton animal counts were determined for each water sample during the two data-collection periods and were identified to the lowest possible taxonomic level.

\section{Vertical Profiles}

At the same time samples were collected at near-bottom and near-surface depths, vertical profiles of $\mathrm{DO}, \mathrm{pH}$, salinity, specific conductance, turbidity, and water temperature were measured at intervals of 1 foot with a multiparameter waterquality monitor. In addition, PAR-sensor profiles, a measure of upwelling and downwelling radiation in a water column between wavelengths of 400 and 700 nanometers, were done in conjunction with the light-dark bottle experiments. Measurements were taken in the air, just above the water surface, at the surface of the water with the sensors just under the water, and then at 1-meter intervals down through the water column. PARsensor measurements in the air were taken to provide an idea of ambient light conditions. The PAR-sensor profiles provide a measurement of sunlight intensity at a specified point in the water column looking toward the water surface (downwelling) and looking toward the streambed (upwelling). Sunlight intensity influences biota in the water column that rely on photosynthesis for growth.

\section{Data Treatment}

Severn Trent Labs (STL) in Denver, Colo., analyzed most of the constituents, except for chlorophyll $a$ and pheophytin $a$, which were analyzed by STL in Westfield, Mass., and particulate carbon and particulate nitrogen, which were analyzed by an STL subcontractor, Nutrient Analytical Services Laboratory, Chesapeake Biological Laboratory in Solomons, Md. Biological analyses were done by USGS laboratory staff, except for phytoplankton analyses, which were done by the Department of Biology and Center for Subtropical Studies, University of Texas-Pan American, Edinburg, Tex.

Environmental sample data were subject to two reviews. Data were initially reviewed by the Contracting Officer's Representative for the USGS analytical-services contract with STL. The focus of this review was to determine the completeness of the samples analyzed, if holding times were met, if dilution factors were applied, and if QA/QC samples were within acceptance criteria. The final data review was done by a designated USGS Data Manager before data were entered into the USGS National Water Information System (NWIS) (U.S. Geological Survey, 2001). The focus of the review was to verify the accuracy and completeness of the laboratory data, to determine if laboratory QA/QC data had any effect on the results, and to determine whether samples were handled appropriately in the field and the laboratory.

Field measurements, such as DO, $\mathrm{pH}$, specific conductance, temperature, and turbidity were reviewed by verifying that the standards used for calibration were adequate, the postcalibration checks were within calibration criteria, and the results were consistent between multiple measurements of the same constituent. The field measured physical characteristics were measured multiple times during instantaneous measurements, vertical profile measurements, and 24-hour continuous monitoring.

Field analyses consisted of BOD determinations and water-column primary productivity measurements (light-dark bottle experiment) (U.S. Geological Survey, 2006). Replicate sample analysis was used to determine the variability of the results. BOD data were qualified in accordance with standard USGS methods (U.S. Geological Survey, 2006).

The field QA/QC samples collected were field blanks and field replicates (two each). Field blanks were used to check for sample contamination in the field by analyzing inorganics-free water that was exposed to the field-sampling equipment used to 
collect and process environmental samples. Field replicates were used to assess the variability of sample handling, preservation, and storage. QA/QC sample results are in appendix 1, table $1-3$.

Data were combined into sample sets organized by site, date, and time following the review process described above. The sample sets were entered into the USGS NWIS, and chemical verification checks were done and used to evaluate each sample set. Chemical verification checks are these: Total constituents concentration must be greater than dissolved constituents concentration, mass ion-balance ratios must be within specified limits of \pm 5.49 percent difference, and the ratio of the sum of constituents to specific conductance must be within specified limits of 0.55 to 0.81 . The final sample sets were checked for transcription and typographical errors.

Finally, data were qualified using "value qualifier codes." Value qualifier codes are used to describe a sample result that does not meet $\mathrm{QA} / \mathrm{QC}$ criteria or to give additional information about how the sample was treated or the analysis was done. The following codes are used: sample diluted (d), value verified by rerun same method $(\mathrm{r})$, sample received warm $(*)$, holding time exceeded (@), and analyte detected in laboratory blank (v). The potential effect of the assignment of the value qualifier code was judged by the USGS Data Manager, and subsequently, a "remark code" was assigned. Samples were assigned a remark code of "less than" $(<)$ if the results were below the laboratory reporting limit. Samples were assigned a remark code of "estimated" (E) if the data values are uncertain because of the deficiencies noted by the value qualifier code(s). For this report, remark codes only are included in the data tables. The value qualifier codes are available in the NWIS database.

\section{Water-Quality and Ancillary Data}

Data are grouped into two appendixes at the end of this report: (1) data collected at the near-surface and near-bottom depths of the Arroyo Colorado and (2) vertical profile data collected in the Arroyo Colorado. Data collected at near-bottom depth of the Arroyo Colorado are listed in the following order (appendixes 1-1.1 through 1-1.4): algal productivity measurements, continuous water-quality monitor measurements, sedimentation measurements, and discrete sample results. Data collected at near-surface depth of the Arroyo Colorado are listed in the following order (appendixes 1-2.1 through 1-2.4): algal productivity measurements, continuous water-quality monitor measurements, algal growth and mortality analyses (bioassay analysis, phytoplankton analysis, zooplankton analysis), and discrete sample results. Discrete sample results (appendixes 1-1.4 and 1-2.4) are categorized by data-collection period. Data collected for QA/QC purposes are listed in appen- dix 1-3. Water-quality vertical profile data are listed in appendix $2-1$, and the PAR sensor vertical profile data are listed in appendix 2-2.

Water-quality data shown in this report allow specific comparisons between the two depths for each of the four sites sampled in the tidal segment of the Arroyo Colorado during the February and May 2006 data-collection periods. Finally, the water-quality data facilitate general analyses of the productivity, biochemical oxygen dynamics, and nutrient cycling in the tidal segment of the Arroyo Colorado, Texas.

\section{References}

American Public Health Association, 1998, Standard methods for the examination of water and wastewater (20th ed.): American Public Health Association, United Book Press, $1,220 \mathrm{p}$.

Lehman, J.T., and Sandgren, C.D., 1985, Species-specific rates of growth and grazing loss among freshwater algae: Limnology Oceanography, v. 30, p. 34-46.

National Oceanic and Atmospheric Administration, 1996, Climatological data, Texas, annual summary: Asheville, N.C., U.S. Department of Commerce, v. 101, no. 13, 77 p.

Raines, T.H., and Miranda, R.M., 2002, Simulation of flow and water quality of the Arroyo Colorado, Texas, 1989-99: U.S. Geological Survey Water-Resources Investigations Report 02-4110, $56 \mathrm{p}$.

Texas Commission on Environmental Quality, 2003, Surface water quality monitoring procedures, Volume 1-Physical and chemical monitoring methods for water, sediment and tissue: Texas Commission on Environmental Quality, RG-415 [variously paged].

Texas Natural Resource Conservation Commission, 1997, Texas surface-water-quality standards: Texas Natural Resource Conservation Commission, chap. 307.1-307.10, $125 \mathrm{p}$.

Wetzel, R.G., and Likens, G.E., 1979, Limnological analyses: Philadelphia, W.B. Saunders Co., 429 p.

U.S. Environmental Protection Agency, 1983, Methods for chemical analysis of water and wastes: U.S. Environmental Protection Agency Report EPA/600/4-79/020.

U.S. Geological Survey, 2001, National Water Information System (NWISWeb) data available on the World Wide Web, accessed January-September 2006 at http://waterdata.usgs.gov/nwis/.

U.S. Geological Survey, 2006, National field manual for the collection of water-quality data: U.S. Geological Survey Techniques of Water-Resources Investigations, book 9, chaps. A1-A9, accessed January-September 2006 at http://pubs.water.usgs.gov/twri9A 
Blank Page 
Appendix 1-Data Collected at Near-Bottom and Near-Surface Depths of the Arroyo Colorado Near Rio Hondo, Texas 
Blank Page 
Appendix 1-1.1. Data collected at near-bottom depth of the Arroyo Colorado near Rio Hondo, Texas, using algal productivity methods (5 feet below water surface).

[mg $\mathrm{O}_{2} / \mathrm{m}^{3} /$ day, milligrams of oxygen per cubic meter per day; negative value signifies deficit of oxygen and positive value signifies accumulation of oxygen]

\begin{tabular}{cclccc}
\hline $\begin{array}{c}\text { USGS } \\
\text { station no. }\end{array}$ & Date & $\begin{array}{c}\text { Data- } \\
\text { collection } \\
\text { period }\end{array}$ & $\begin{array}{c}\text { Gross primary } \\
\text { productivity } \\
\text { (mg 0 } \mathrm{m}_{2} / \mathrm{m}^{3} / \text { day) }\end{array}$ & $\begin{array}{c}\text { Net primary } \\
\text { productivity } \\
\left(\mathrm{mg} \mathrm{O}_{2} / \mathrm{m}^{3} / \text { day) }\right.\end{array}$ & $\begin{array}{c}\text { Respiration } \\
\left(\mathrm{mg} \mathrm{O}_{2} / \mathrm{m}^{3} / \text { day }\right)\end{array}$ \\
\hline 08470460 & $02 / 22 / 2006$ & February & 568 & $-2,309$ & 2,878 \\
08470460 & $05 / 23 / 2006$ & May & 2,626 & $-5,082$ & 7,708 \\
08470500 & $02 / 22 / 2006$ & February & 51 & $-5,788$ & 5,839 \\
08470500 & $05 / 23 / 2006$ & May & 11,560 & 9,560 & 2,400 \\
\hline
\end{tabular}

Appendix 1-1.2. Data collected at near-bottom depth of the Arroyo Colorado near Rio Hondo, Texas, using multiparameter continuous water-quality monitors.

$\left[{ }^{\circ} \mathrm{C}\right.$, degrees Celsius; $\mu \mathrm{S} / \mathrm{cm}$, microsiemens per centimeter at 25 degrees Celsius; $\mathrm{mg} / \mathrm{L}$, milligrams per liter. Dissolved oxygen values less than $2.0 \mathrm{mg} / \mathrm{L}$ reported here to show resolution in a low dissolved oxygen environment, rather than set all values to $<2.0 \mathrm{mg} / \mathrm{L}$; zero values should be interpreted as $<0.1 \mathrm{mg} / \mathrm{L}$ ]

\begin{tabular}{|c|c|c|c|c|c|c|c|c|c|c|c|}
\hline $\begin{array}{c}\text { USGS } \\
\text { station } \\
\text { no. }\end{array}$ & $\begin{array}{l}\text { Date and } \\
\text { time }\end{array}$ & $\begin{array}{c}\text { Temp- } \\
\text { erature } \\
\left({ }^{\circ} \mathrm{C}\right)\end{array}$ & $\begin{array}{c}\text { Specific } \\
\text { conduc- } \\
\text { tance } \\
(\mu \mathrm{S} / \mathrm{cm})\end{array}$ & $\begin{array}{l}\text { Dis- } \\
\text { solved } \\
\text { oxygen } \\
\text { (mg/L) }\end{array}$ & $\begin{array}{c}\mathrm{pH} \\
\text { (stan- } \\
\text { dard } \\
\text { units) }\end{array}$ & $\begin{array}{c}\text { USGS } \\
\text { station } \\
\text { no. }\end{array}$ & $\begin{array}{l}\text { Date and } \\
\text { time }\end{array}$ & $\begin{array}{l}\text { Temp- } \\
\text { erature } \\
\left({ }^{\circ} \mathrm{C}\right)\end{array}$ & $\begin{array}{c}\text { Specific } \\
\text { conduc- } \\
\text { tance } \\
(\mu \mathrm{S} / \mathrm{cm})\end{array}$ & $\begin{array}{c}\text { Dis- } \\
\text { solved } \\
\text { oxygen } \\
\text { (mg/L) }\end{array}$ & $\begin{array}{c}\mathrm{pH} \\
\text { (stan- } \\
\text { dard } \\
\text { units) }\end{array}$ \\
\hline 08470450 & $02 / 22 / 2006$ 10:30 & 17.8 & 3,500 & 7.6 & 7.5 & 08470450 & 02/22/2006 20:00 & 19.0 & 9,980 & 6.1 & 7.7 \\
\hline 08470450 & 02/22/2006 10:45 & 17.8 & 3,510 & 7.7 & 7.6 & 08470450 & 02/22/2006 20:15 & 19.0 & 11,200 & 5.9 & 7.7 \\
\hline 08470450 & 02/22/2006 11:00 & 18.1 & 3,440 & 7.8 & 7.6 & 08470450 & 02/22/2006 20:30 & 19.0 & 10,400 & 6.4 & 7.7 \\
\hline 08470450 & $02 / 22 / 200611: 45$ & 18.2 & 3,460 & 7.8 & 7.6 & 08470450 & 02/22/2006 21:15 & 19.2 & 8,070 & 7.0 & 7.6 \\
\hline 08470450 & 02/22/2006 12:00 & 18.2 & 3,490 & 7.8 & 7.6 & 08470450 & $02 / 22 / 200621: 30$ & 19.2 & 7,550 & 7.2 & 7.6 \\
\hline 08470450 & $02 / 22 / 200612: 15$ & 18.3 & 3,500 & 7.9 & 7.6 & 08470450 & $02 / 22 / 200621: 45$ & 19.3 & 6,200 & 7.2 & 7.7 \\
\hline 08470450 & $02 / 22 / 200612: 30$ & 18.4 & 3,490 & 7.8 & 7.6 & 08470450 & 02/22/2006 22:00 & 19.3 & 6,050 & 7.2 & 7.6 \\
\hline 08470450 & $02 / 22 / 200612: 45$ & 18.4 & 3,510 & 7.8 & 7.6 & 08470450 & 02/22/2006 22:15 & 19.4 & 5,420 & 7.3 & 7.7 \\
\hline 08470450 & 02/22/2006 13:00 & 18.6 & 3,500 & 7.9 & 7.6 & 08470450 & $02 / 22 / 200622: 30$ & 19.4 & 4,690 & 7.5 & 7.7 \\
\hline 08470450 & 02/22/2006 14:15 & 19.0 & 3,500 & 8.0 & 7.7 & 08470450 & 02/22/2006 23:45 & 19.6 & 3,730 & 7.5 & 7.7 \\
\hline 08470450 & $02 / 22 / 200614: 30$ & 19.0 & 3,520 & 8.0 & 7.6 & 08470450 & 02/23/2006 00:00 & 19.6 & 3,730 & 7.4 & 7.7 \\
\hline 08470450 & 02/22/2006 14:45 & 19.0 & 3,530 & 8.1 & 7.7 & 08470450 & 02/23/2006 00:15 & 19.7 & 3,740 & 7.5 & 7.7 \\
\hline 08470450 & 02/22/2006 15:00 & 19.5 & 3,460 & 8.1 & 7.7 & 08470450 & 02/23/2006 00:30 & 19.7 & 3,740 & 7.4 & 7.7 \\
\hline 08470450 & $02 / 22 / 200615: 15$ & 19.4 & 3,490 & 8.0 & 7.7 & 08470450 & 02/23/2006 00:45 & 19.8 & 3,740 & 7.3 & 7.7 \\
\hline 08470450 & $02 / 22 / 200615: 30$ & 19.5 & 3,500 & 8.0 & 7.7 & 08470450 & 02/23/2006 01:00 & 19.8 & 3,750 & 7.3 & 7.6 \\
\hline 08470450 & $02 / 22 / 200615: 45$ & 19.4 & 3,530 & 8.0 & 7.7 & 08470450 & 02/23/2006 01:15 & 19.8 & 3,740 & 7.3 & 7.7 \\
\hline 08470450 & 02/22/2006 16:00 & 19.5 & 3,530 & 8.1 & 7.7 & 08470450 & 02/23/2006 01:30 & 19.9 & 3,750 & 7.3 & 7.6 \\
\hline 08470450 & $02 / 22 / 200616: 15$ & 19.6 & 3,520 & 8.1 & 7.7 & 08470450 & 02/23/2006 01:45 & 19.9 & 3,750 & 7.3 & 7.6 \\
\hline 08470450 & $02 / 22 / 200616: 30$ & 19.6 & 3,540 & 8.0 & 7.7 & 08470450 & 02/23/2006 02:00 & 19.9 & 3,750 & 7.3 & 7.6 \\
\hline 08470450 & $02 / 22 / 200616: 45$ & 19.6 & 3,900 & 8.0 & 7.7 & 08470450 & 02/23/2006 02:15 & 20.0 & 3,750 & 7.2 & 7.6 \\
\hline 08470450 & 02/22/2006 19:00 & 18.8 & 9,380 & 5.5 & 7.7 & 08470450 & 02/23/2006 04:30 & 20.3 & 3,720 & 7.1 & 7.6 \\
\hline 08470450 & $02 / 22 / 200619: 15$ & 19.0 & 8,610 & 5.7 & 7.7 & 08470450 & 02/23/2006 04:45 & 20.3 & 3,720 & 7.0 & 7.6 \\
\hline 08470450 & 02/22/2006 19:30 & 19.0 & 9,450 & 6.1 & 7.6 & 08470450 & 02/23/2006 05:00 & 20.3 & 3,710 & 7.0 & 7.6 \\
\hline 08470450 & 02/22/2006 19:45 & 19.0 & 10,100 & 6.2 & 7.6 & 08470450 & 02/23/2006 05:15 & 20.3 & 3,710 & 7.0 & 7.6 \\
\hline
\end{tabular}


Appendix 1-1.2. Data collected at near-bottom depth of the Arroyo Colorado near Rio Hondo, Texas, using multiparameter continuous water-quality monitors-Continued.

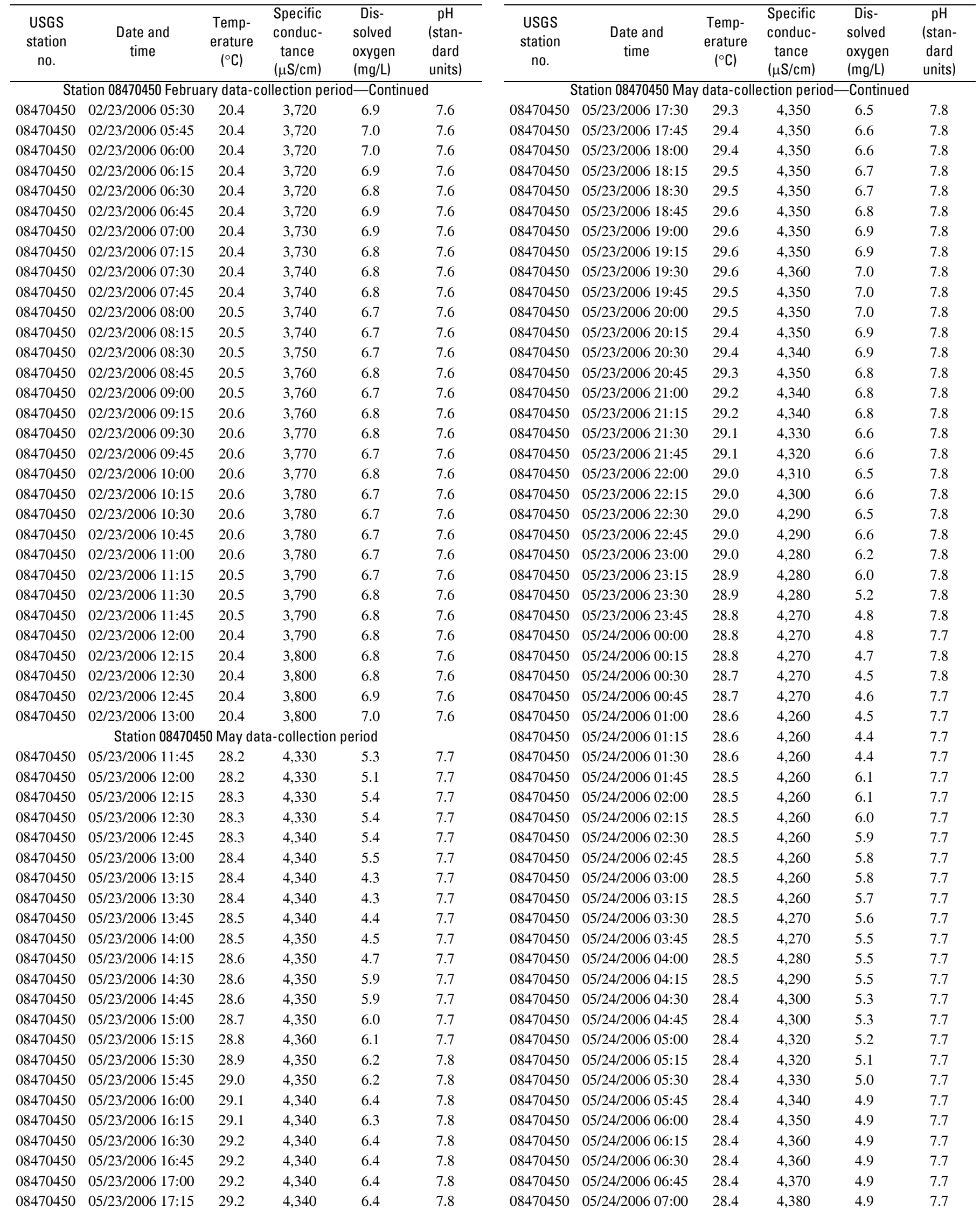


Appendix 1-1.2. Data collected at near-bottom depth of the Arroyo Colorado near Rio Hondo, Texas, using multiparameter continuous water-quality monitors-Continued.

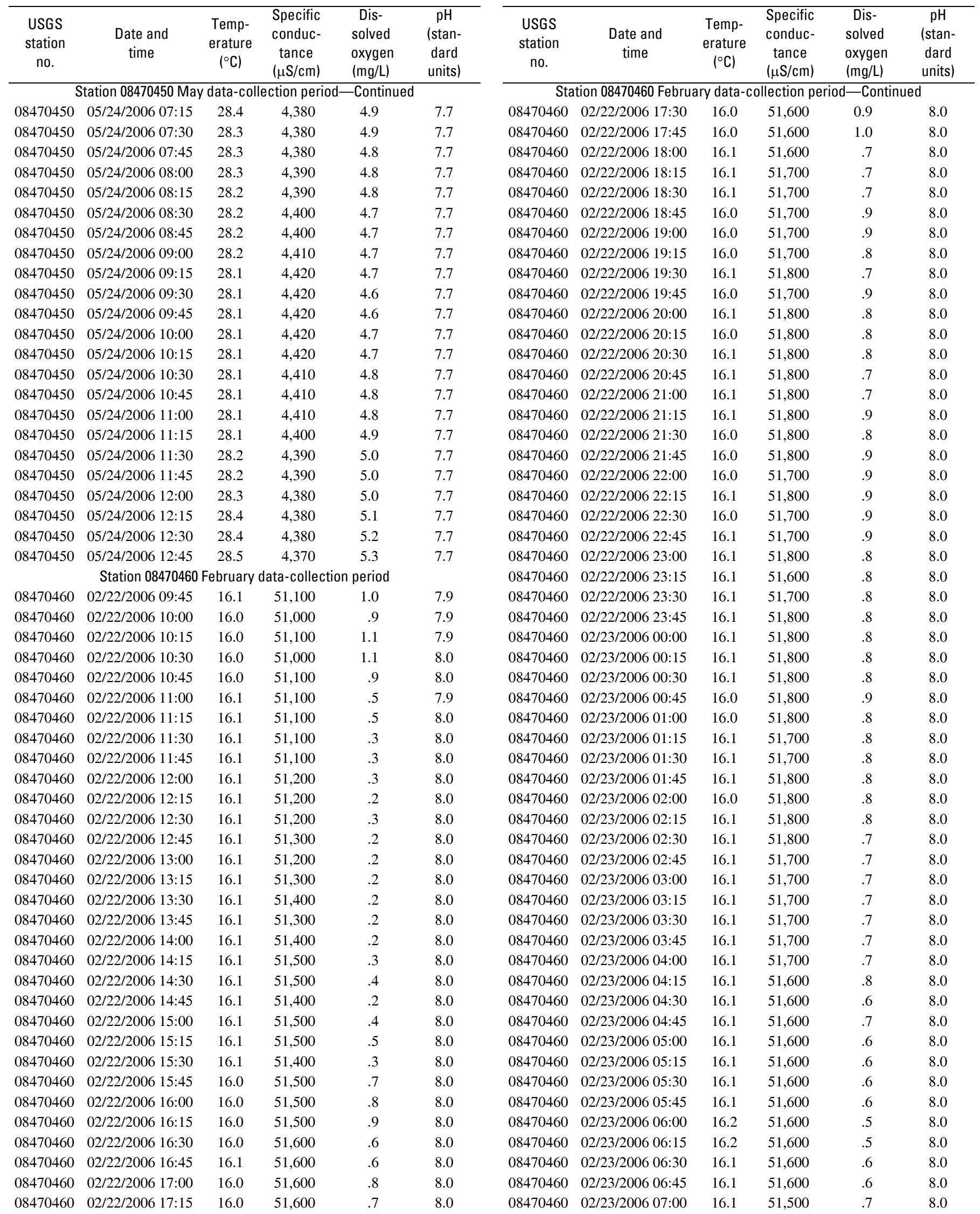


Appendix 1-1.2. Data collected at near-bottom depth of the Arroyo Colorado near Rio Hondo, Texas, using multiparameter continuous water-quality monitors-Continued.

\begin{tabular}{|c|c|c|c|c|c|c|c|c|c|c|c|}
\hline $\begin{array}{l}\text { USGS } \\
\text { station } \\
\text { no. }\end{array}$ & $\begin{array}{c}\text { Date and } \\
\text { time }\end{array}$ & $\begin{array}{l}\text { Temp- } \\
\text { erature } \\
\left({ }^{\circ} \mathrm{C}\right)\end{array}$ & $\begin{array}{c}\text { Specific } \\
\text { conduc- } \\
\text { tance } \\
(\mu \mathrm{S} / \mathrm{cm})\end{array}$ & $\begin{array}{c}\text { Dis- } \\
\text { solved } \\
\text { oxygen } \\
\text { (mg/L) }\end{array}$ & $\begin{array}{c}\mathrm{pH} \\
\text { (stan- } \\
\text { dard } \\
\text { units) }\end{array}$ & $\begin{array}{l}\text { USGS } \\
\text { station } \\
\text { no. }\end{array}$ & $\begin{array}{c}\text { Date and } \\
\text { time }\end{array}$ & $\begin{array}{l}\text { Temp- } \\
\text { erature } \\
\left({ }^{\circ} \mathrm{C}\right)\end{array}$ & $\begin{array}{c}\text { Specific } \\
\text { conduc- } \\
\text { tance } \\
(\mu \mathrm{S} / \mathrm{cm})\end{array}$ & $\begin{array}{c}\text { Dis- } \\
\text { solved } \\
\text { oxygen } \\
\text { (mg/L) }\end{array}$ & $\begin{array}{c}\mathrm{pH} \\
\text { (stan- } \\
\text { dard } \\
\text { units) }\end{array}$ \\
\hline \multicolumn{6}{|c|}{ Station 08470460 February data-collection period-Continued } & \multicolumn{6}{|c|}{ Station 08470460 May data-collection period-Continued } \\
\hline 08470460 & 02/23/2006 07:15 & 16.1 & 51,500 & 0.6 & 8.0 & 08470460 & 05/23/2006 18:00 & 27.0 & 23,300 & 0.1 & 6.9 \\
\hline 08470460 & 02/23/2006 07:30 & 16.1 & 51,600 & .7 & 8.0 & 08470460 & 05/23/2006 18:15 & 26.9 & 23,300 & .1 & 6.9 \\
\hline 08470460 & $02 / 23 / 200607: 45$ & 16.1 & 51,500 & .6 & 8.0 & 08470460 & 05/23/2006 18:30 & 26.9 & 23,200 & .1 & 6.9 \\
\hline 08470460 & 02/23/2006 08:30 & 16.1 & 51,600 & .6 & 8.0 & 08470460 & 05/23/2006 19:15 & 26.9 & 23,200 & .1 & 6.9 \\
\hline 08470460 & 02/23/2006 08:45 & 16.1 & 51,600 & 6 & 8.0 & 08470460 & 05/23/2006 19:30 & 26.9 & 23,100 & .1 & 6.9 \\
\hline 08470460 & 02/23/2006 09:00 & 16.1 & 51,600 & 6 & 8.0 & 08470460 & 05/23/2006 19:45 & 26.9 & 23,100 & .1 & 6.9 \\
\hline 08470460 & 02/23/2006 09:15 & 16.1 & 51,600 & .5 & 8.0 & 08470460 & 05/23/2006 20:00 & 26.9 & 23,100 & .1 & 6.9 \\
\hline 08470460 & 02/23/2006 09:30 & 16.1 & 51,600 & .5 & 8.0 & 08470460 & 05/23/2006 20:15 & 26.9 & 23,100 & .1 & 6.9 \\
\hline 08470460 & 02/23/2006 09:45 & 16.1 & 51,700 & .5 & 8.0 & 08470460 & 05/23/2006 20:30 & 26.9 & 23,100 & .1 & 6.9 \\
\hline 08470460 & 02/23/2006 11:00 & 16.2 & 51,400 & .5 & 8.0 & 08470460 & 05/23/2006 21:45 & 26.8 & 23,100 & .1 & 6.9 \\
\hline 08470460 & 02/23/2006 11:15 & 16.2 & 51,300 & .4 & 8.0 & 08470460 & 05/23/2006 22:00 & 26.8 & 23,100 & .1 & 6.9 \\
\hline 08470460 & 02/23/2006 11:30 & 16.2 & 51,100 & .4 & 8.0 & 08470460 & 05/23/2006 22:15 & 26.8 & 23,100 & .1 & 6.9 \\
\hline 08470460 & 02/23/2006 11:45 & 16.2 & 51,100 & .5 & 8.0 & 08470460 & $05 / 23 / 200622: 30$ & 26.8 & 23,100 & .1 & 6.9 \\
\hline 08470460 & 02/23/2006 12:00 & 16.2 & 50,700 & .4 & 8.0 & 08470460 & 05/23/2006 22:45 & 26.8 & 23,100 & .1 & 6.9 \\
\hline 08470460 & 02/23/2006 12:15 & 16.3 & 50,600 & .4 & 8.0 & 08470460 & 05/23/2006 23:00 & 26.8 & 23,100 & .1 & 6.9 \\
\hline 08470460 & 02/23/2006 12:30 & 16.2 & 51,100 & .4 & 8.0 & 08470460 & $05 / 23 / 200623: 15$ & 26.8 & 23,100 & .1 & 6.9 \\
\hline 08470460 & $02 / 23 / 200612: 45$ & 16.3 & 50,600 & .5 & 8.0 & 08470460 & 05/23/2006 23:30 & 26.8 & 23,100 & .1 & 6.9 \\
\hline 08470460 & 02/23/2006 13:00 & 16.3 & 50,900 & .4 & 8.0 & 08470460 & 05/23/2006 23:45 & 26.8 & 23,100 & .1 & 6.9 \\
\hline 08470460 & 02/23/2006 13:15 & 16.3 & 50,600 & .4 & 8.0 & 08470460 & 05/24/2006 00:00 & 26.8 & 23,100 & .1 & 6.9 \\
\hline 08470460 & $02 / 23 / 200613: 30$ & 16.3 & 50,500 & .6 & 8.0 & 08470460 & 05/24/2006 00:15 & 26.8 & 23,100 & .1 & 6.9 \\
\hline \multicolumn{6}{|c|}{ Station 08470460 May data-collection period } & 08470460 & 05/24/2006 00:45 & 26.8 & 23,100 & .1 & 6.9 \\
\hline 08470460 & 05/23/2006 11:15 & 26.6 & 24,300 & .2 & 6.9 & 08470460 & 05/24/2006 01:00 & 26.8 & 23,100 & 0 & 6.9 \\
\hline 08470460 & 05/23/2006 11:30 & 26.6 & 23,900 & .1 & 6.9 & 08470460 & 05/24/2006 01:15 & 26.8 & 23,100 & .1 & 6.9 \\
\hline 08470460 & 05/23/2006 11:45 & 26.6 & 23,900 & .1 & 6.9 & 08470460 & 05/24/2006 01:30 & 26.8 & 23,100 & .1 & 6.9 \\
\hline 08470460 & 05/23/2006 12:00 & 26.6 & 23,900 & .1 & 6.9 & 08470460 & 05/24/2006 01:45 & 26.8 & 23,100 & .1 & 6.9 \\
\hline 08470460 & 05/23/2006 12:15 & 26.6 & 23,800 & .1 & 6.9 & 08470460 & 05/24/2006 02:00 & 26.8 & 23,100 & .1 & 6.9 \\
\hline 08470460 & 05/23/2006 12:30 & 26.6 & 23,800 & .1 & 6.9 & 08470460 & 05/24/2006 02:15 & 26.8 & 23,100 & .1 & 6.9 \\
\hline 08470460 & 05/23/2006 12:45 & 26.6 & 23,800 & .1 & 6.9 & 08470460 & 05/24/2006 02:30 & 26.8 & 23,100 & .1 & 6.9 \\
\hline 08470460 & 05/23/2006 13:00 & 26.6 & 23,800 & .1 & 6.9 & 08470460 & 05/24/2006 02:45 & 26.8 & 23,100 & .1 & 6.9 \\
\hline 08470460 & 05/23/2006 13:15 & 26.6 & 23,800 & .1 & 6.9 & 08470460 & 05/24/2006 03:00 & 26.8 & 23,100 & .1 & 6.9 \\
\hline 08470460 & 05/23/2006 13:30 & 26.6 & 23,800 & .1 & 6.9 & 08470460 & 05/24/2006 03:15 & 26.8 & 23,100 & 0 & 6.9 \\
\hline 08470460 & 05/23/2006 13:45 & 26.6 & 23,800 & .1 & 6.9 & 08470460 & 05/24/2006 03:30 & 26.8 & 23,100 & .1 & 6.9 \\
\hline 08470460 & 05/23/2006 14:00 & 26.6 & 23,800 & .1 & 6.9 & 08470460 & 05/24/2006 03:45 & 26.8 & 23,100 & 0 & 6.9 \\
\hline 08470460 & 05/23/2006 14:15 & 26.6 & 23,900 & .1 & 6.9 & 08470460 & 05/24/2006 04:00 & 26.8 & 23,100 & 0 & 6.9 \\
\hline 08470460 & 05/23/2006 14:30 & 26.6 & 23,800 & .1 & 6.9 & 08470460 & 05/24/2006 04:15 & 26.8 & 23,100 & 0 & 6.9 \\
\hline 08470460 & 05/23/2006 14:45 & 26.6 & 23,800 & .1 & 6.9 & 08470460 & 05/24/2006 04:30 & 26.8 & 23,200 & 0 & 6.9 \\
\hline 08470460 & 05/23/2006 15:00 & 26.6 & 23,800 & .1 & 6.9 & 08470460 & 05/24/2006 04:45 & 26.8 & 23,100 & .1 & 6.9 \\
\hline 08470460 & 05/23/2006 15:15 & 26.6 & 23,700 & .1 & 6.9 & 08470460 & 05/24/2006 05:00 & 26.8 & 23,100 & .1 & 6.9 \\
\hline 08470460 & 05/23/2006 15:30 & 26.6 & 23,700 & .1 & 6.9 & 08470460 & 05/24/2006 05:15 & 26.8 & 23,100 & 0 & 6.9 \\
\hline 08470460 & 05/23/2006 15:45 & 26.6 & 23,700 & .1 & 6.9 & 08470460 & 05/24/2006 05:30 & 26.8 & 23,100 & .1 & 6.9 \\
\hline 08470460 & 05/23/2006 16:00 & 27.0 & 23,500 & .1 & 6.9 & 08470460 & 05/24/2006 05:45 & 26.8 & 23,100 & 0 & 6.9 \\
\hline 08470460 & 05/23/2006 16:15 & 27.0 & 23,700 & .1 & 6.9 & 08470460 & 05/24/2006 06:00 & 26.8 & 23,100 & .1 & 6.9 \\
\hline 08470460 & 05/23/2006 16:30 & 27.1 & 23,600 & .1 & 6.9 & 08470460 & 05/24/2006 06:15 & 26.8 & 23,100 & 0 & 6.9 \\
\hline 08470460 & 05/23/2006 16:45 & 27.1 & 23,500 & .1 & 6.9 & 08470460 & 05/24/2006 06:30 & 26.8 & 23,100 & .1 & 6.9 \\
\hline 08470460 & 05/23/2006 17:00 & 27.4 & 23,500 & .1 & 6.9 & 08470460 & 05/24/2006 06:45 & 26.8 & 23,100 & .1 & 6.9 \\
\hline 08470460 & 05/23/2006 17:15 & 26.8 & 23,500 & .1 & 6.9 & 08470460 & 05/24/2006 07:00 & 26.8 & 23,100 & .1 & 6.9 \\
\hline 08470460 & 05/23/2006 17:30 & 26.8 & 23,400 & .1 & 6.9 & 08470460 & 05/24/2006 07:15 & 26.7 & 23,200 & .1 & 6.9 \\
\hline 08470460 & 05/23/2006 17:45 & 27.0 & 23,400 & .1 & 6.9 & 08470460 & 05/24/2006 07:30 & 26.7 & 23,100 & 0 & 6.9 \\
\hline
\end{tabular}


Appendix 1-1.2. Data collected at near-bottom depth of the Arroyo Colorado near Rio Hondo, Texas, using multiparameter continuous water-quality monitors-Continued.

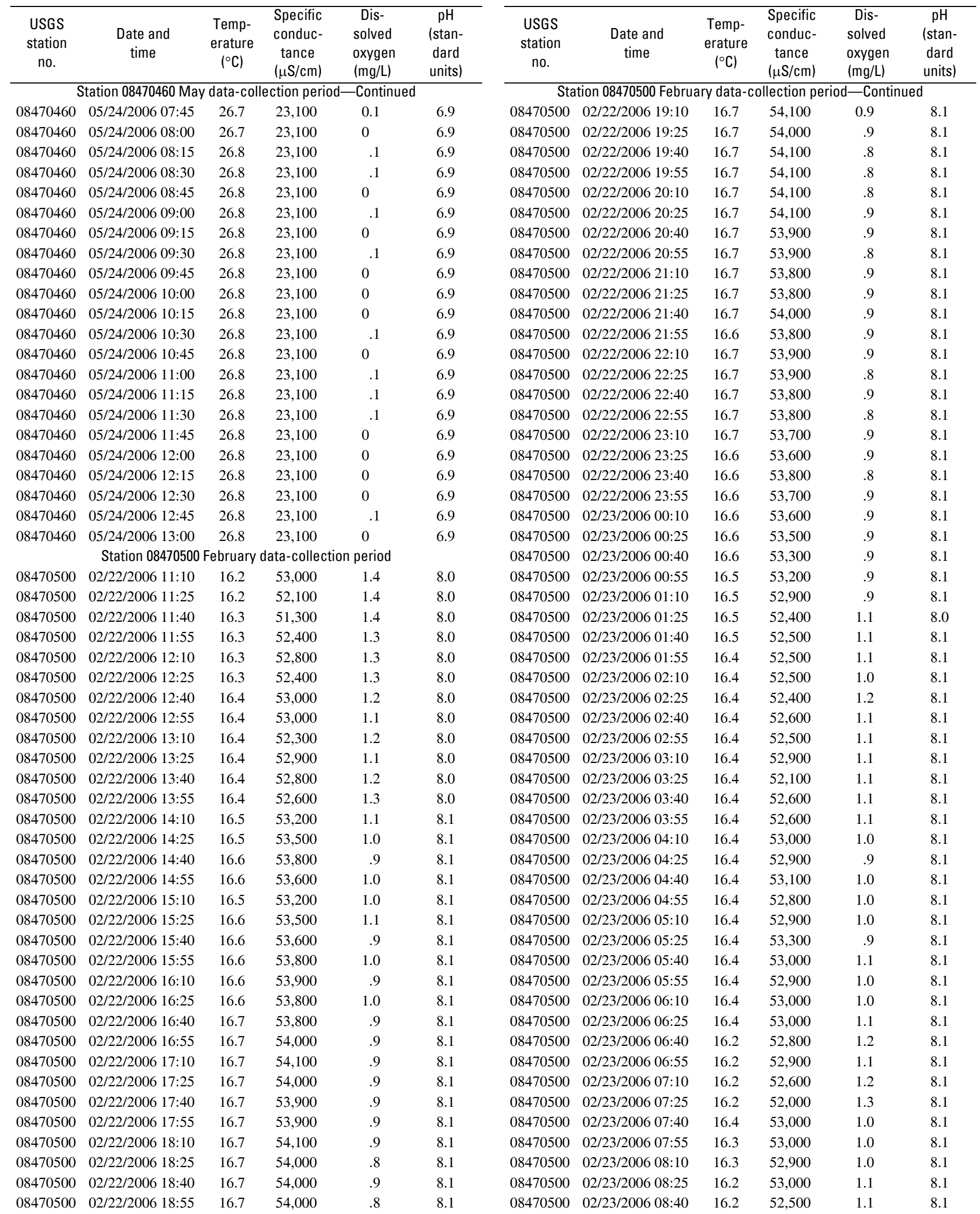


Appendix 1-1.2. Data collected at near-bottom depth of the Arroyo Colorado near Rio Hondo, Texas, using multiparameter continuous water-quality monitors-Continued.

\begin{tabular}{|c|c|c|c|c|c|c|c|c|c|c|c|}
\hline $\begin{array}{l}\text { USGS } \\
\text { station } \\
\text { no. }\end{array}$ & $\begin{array}{l}\text { Date and } \\
\text { time }\end{array}$ & $\begin{array}{c}\text { Temp- } \\
\text { erature } \\
\left({ }^{\circ} \mathrm{C}\right)\end{array}$ & $\begin{array}{c}\text { Specific } \\
\text { conduc- } \\
\text { tance } \\
(\mu \mathrm{S} / \mathrm{cm})\end{array}$ & $\begin{array}{l}\begin{array}{c}\text { Dis- } \\
\text { solved } \\
\text { oxygen } \\
(\mathrm{mg} / \mathrm{L})\end{array} \\
\end{array}$ & $\begin{array}{c}\mathrm{pH} \\
\text { (stan- } \\
\text { dard } \\
\text { units) }\end{array}$ & $\begin{array}{l}\text { USGS } \\
\text { station } \\
\text { no. }\end{array}$ & $\begin{array}{c}\text { Date and } \\
\text { time }\end{array}$ & $\begin{array}{l}\text { Temp- } \\
\text { erature } \\
\left({ }^{\circ} \mathrm{C}\right)\end{array}$ & $\begin{array}{c}\text { Specific } \\
\text { conduc- } \\
\text { tance } \\
(\mu \mathrm{S} / \mathrm{cm})\end{array}$ & $\begin{array}{c}\text { Dis- } \\
\text { solved } \\
\text { oxygen } \\
\text { (mg/L) }\end{array}$ & $\begin{array}{c}\mathrm{pH} \\
\text { (stan- } \\
\text { dard } \\
\text { units) }\end{array}$ \\
\hline \multicolumn{6}{|c|}{ Station 08470500 February data-collection period-Continued } & \multicolumn{6}{|c|}{ Station 08470500 May data-collection period-Continued } \\
\hline 08470500 & 02/23/2006 08:55 & 16.1 & 52,300 & 1.2 & 8.1 & 08470500 & 05/23/2006 18:30 & 26.7 & 34,300 & 0.1 & 7.6 \\
\hline 08470500 & 02/23/2006 09:10 & 16.2 & 52,500 & 1.1 & 8.1 & 08470500 & 05/23/2006 18:45 & 26.7 & 33,700 & .1 & 7.6 \\
\hline 08470500 & 02/23/2006 09:25 & 16.1 & 51,700 & 1.3 & 8.0 & 08470500 & 05/23/2006 19:00 & 26.7 & 33,600 & .1 & 7.6 \\
\hline 08470500 & 02/23/2006 10:10 & 15.9 & 51,600 & 1.6 & 8.0 & 08470500 & 05/23/2006 19:45 & 26.7 & 34,000 & .1 & 7.6 \\
\hline 08470500 & 02/23/2006 10:25 & 16.0 & 51,500 & 1.5 & 8.0 & 08470500 & 05/23/2006 20:00 & 26.7 & 33,700 & .1 & 7.6 \\
\hline 08470500 & 02/23/2006 10:40 & 16.0 & 51,100 & 1.6 & 8.0 & 08470500 & 05/23/2006 20:15 & 26.7 & 33,800 & .1 & 7.6 \\
\hline 08470500 & 02/23/2006 10:55 & 16.1 & 51,700 & 1.4 & 8.0 & 08470500 & 05/23/2006 20:30 & 26.7 & 33,300 & .1 & 7.6 \\
\hline 08470500 & 02/23/2006 11:10 & 15.9 & 51,000 & 1.6 & 8.0 & 08470500 & 05/23/2006 20:45 & 26.7 & 33,200 & .1 & 7.6 \\
\hline 08470500 & 02/23/2006 11:25 & 16.0 & 50,900 & 1.3 & 8.0 & 08470500 & 05/23/2006 21:00 & 26.8 & 30,400 & .1 & 7.6 \\
\hline 08470500 & 02/23/2006 12:40 & 16.1 & 50,800 & 1.1 & 8.0 & 08470500 & 05/23/2006 22:15 & 26.8 & 30,300 & .1 & 7.6 \\
\hline 08470500 & 02/23/2006 12:55 & 16.2 & 50,800 & 1.1 & 8.0 & 08470500 & 05/23/2006 22:30 & 26.8 & 30,300 & .1 & 7.6 \\
\hline 08470500 & 02/23/2006 13:10 & 16.3 & 50,300 & 1.0 & 8.0 & 08470500 & 05/23/2006 22:45 & 26.8 & 30,100 & .1 & 7.6 \\
\hline 08470500 & 02/23/2006 13:25 & 16.3 & 50,400 & 1.0 & 8.0 & 08470500 & 05/23/2006 23:00 & 27.0 & 27,700 & .1 & 7.6 \\
\hline 08470500 & 02/23/2006 13:40 & 16.4 & 49,700 & 1.0 & 8.0 & 08470500 & 05/23/2006 23:15 & 27.1 & 26,100 & .1 & 7.6 \\
\hline 08470500 & 02/23/2006 13:55 & 16.6 & 49,200 & .9 & 8.0 & 08470500 & 05/23/2006 23:30 & 27.0 & 26,900 & .1 & 7.6 \\
\hline 08470500 & 02/23/2006 14:10 & 16.5 & 49,600 & .9 & 8.0 & 08470500 & 05/23/2006 23:45 & 27.2 & 24,700 & .1 & 7.5 \\
\hline 08470500 & $02 / 23 / 200614: 25$ & 16.4 & 50,000 & .9 & 8.0 & 08470500 & 05/24/2006 00:00 & 27.2 & 24,700 & .1 & 7.5 \\
\hline \multicolumn{6}{|c|}{ Station 08470500 May data-collection period } & 08470500 & 05/24/2006 00:15 & 27.2 & 24,700 & .1 & 7.5 \\
\hline 08470500 & 05/23/2006 10:45 & 26.7 & 34,200 & .2 & 7.6 & 08470500 & 05/24/2006 00:30 & 27.1 & 26,000 & .1 & 7.5 \\
\hline 08470500 & 05/23/2006 11:00 & 26.7 & 33,900 & .2 & 7.6 & 08470500 & 05/24/2006 00:45 & 27.2 & 24,800 & .1 & 7.5 \\
\hline 08470500 & 05/23/2006 13:15 & 26.7 & 33,700 & .1 & 7.6 & 08470500 & 05/24/2006 03:00 & 26.8 & 32,200 & .1 & 7.6 \\
\hline 08470500 & 05/23/2006 13:30 & 26.7 & 33,600 & .1 & 7.6 & 08470500 & 05/24/2006 03:15 & 26.7 & 32,400 & .1 & 7.6 \\
\hline 08470500 & 05/23/2006 13:45 & 26.7 & 33,300 & .1 & 7.6 & 08470500 & 05/24/2006 03:30 & 26.7 & 32,700 & .1 & 7.6 \\
\hline 08470500 & 05/23/2006 14:00 & 26.7 & 33,000 & .1 & 7.6 & 08470500 & 05/24/2006 03:45 & 26.7 & 32,500 & .1 & 7.6 \\
\hline 08470500 & 05/23/2006 14:15 & 26.7 & 32,700 & .1 & 7.6 & 08470500 & 05/24/2006 04:00 & 26.7 & 34,200 & .1 & 7.6 \\
\hline 08470500 & 05/23/2006 14:30 & 26.7 & 33,100 & .2 & 7.6 & 08470500 & 05/24/2006 04:15 & 26.7 & 32,400 & .1 & 7.6 \\
\hline 08470500 & 05/23/2006 14:45 & 26.7 & 33,300 & .2 & 7.6 & 08470500 & 05/24/2006 04:30 & 26.7 & 33,600 & .1 & 7.6 \\
\hline 08470500 & 05/23/2006 15:00 & 26.7 & 33,300 & .1 & 7.6 & 08470500 & 05/24/2006 04:45 & 26.7 & 33,200 & .1 & 7.6 \\
\hline 08470500 & 05/23/2006 15:15 & 26.7 & 33,400 & .1 & 7.3 & 08470500 & 05/24/2006 05:00 & 26.6 & 34,300 & .1 & 7.6 \\
\hline 08470500 & 05/23/2006 15:30 & 26.7 & 33,100 & .1 & 7.6 & 08470500 & 05/24/2006 05:15 & 26.7 & 32,900 & .1 & 7.6 \\
\hline 08470500 & 05/23/2006 15:45 & 26.7 & 33,100 & .1 & 7.6 & 08470500 & 05/24/2006 05:30 & 26.8 & 30,400 & .1 & 7.6 \\
\hline 08470500 & 05/23/2006 16:00 & 26.7 & 33,000 & .1 & 7.6 & 08470500 & 05/24/2006 05:45 & 26.9 & 30,200 & .1 & 7.6 \\
\hline 08470500 & 05/23/2006 16:15 & 26.7 & 33,800 & .1 & 7.6 & 08470500 & 05/24/2006 06:00 & 27.0 & 29,200 & .1 & 7.6 \\
\hline 08470500 & 05/23/2006 16:30 & 26.7 & 33,900 & .1 & 7.6 & 08470500 & 05/24/2006 06:15 & 26.9 & 29,800 & .1 & 7.6 \\
\hline 08470500 & 05/23/2006 16:45 & 26.7 & 33,200 & .1 & 7.6 & 08470500 & 05/24/2006 06:30 & 26.8 & 31,800 & .1 & 7.6 \\
\hline 08470500 & 05/23/2006 17:00 & 26.7 & 33,500 & .1 & 7.6 & 08470500 & 05/24/2006 06:45 & 26.8 & 31,900 & .1 & 7.6 \\
\hline 08470500 & 05/23/2006 17:15 & 26.7 & 34,000 & .1 & 7.6 & 08470500 & 05/24/2006 07:00 & 26.8 & 31,500 & .1 & 7.6 \\
\hline 08470500 & 05/23/2006 17:30 & 26.7 & 34,000 & .1 & 7.6 & 08470500 & 05/24/2006 07:15 & 26.8 & 31,900 & .1 & 7.6 \\
\hline 08470500 & 05/23/2006 17:45 & 26.7 & 33,800 & .1 & 7.6 & 08470500 & 05/24/2006 07:30 & 26.8 & 31,300 & .1 & 7.6 \\
\hline 08470500 & 05/23/2006 18:00 & 26.7 & 33,800 & .1 & 7.6 & 08470500 & 05/24/2006 07:45 & 26.8 & 31,300 & .1 & 7.6 \\
\hline 08470500 & 05/23/2006 18:15 & 26.7 & 33,600 & .1 & 7.6 & 08470500 & 05/24/2006 08:00 & 26.7 & 31,800 & .1 & 7.5 \\
\hline
\end{tabular}


Appendix 1-1.2. Data collected at near-bottom depth of the Arroyo Colorado near Rio Hondo, Texas, using multiparameter continuous water-quality monitors-Continued.

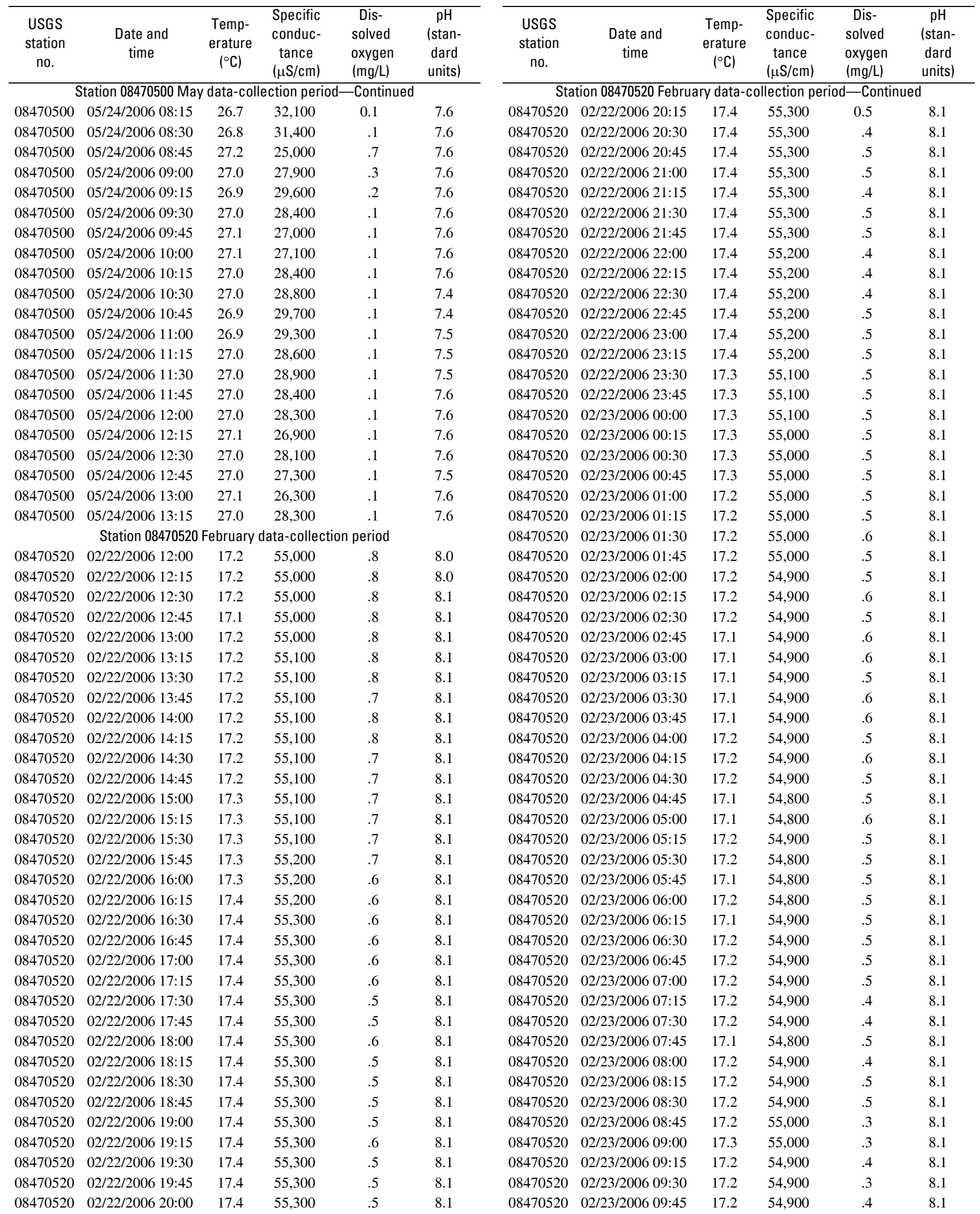


Appendix 1-1.2. Data collected at near-bottom depth of the Arroyo Colorado near Rio Hondo, Texas, using multiparameter continuous water-quality monitors-Continued.

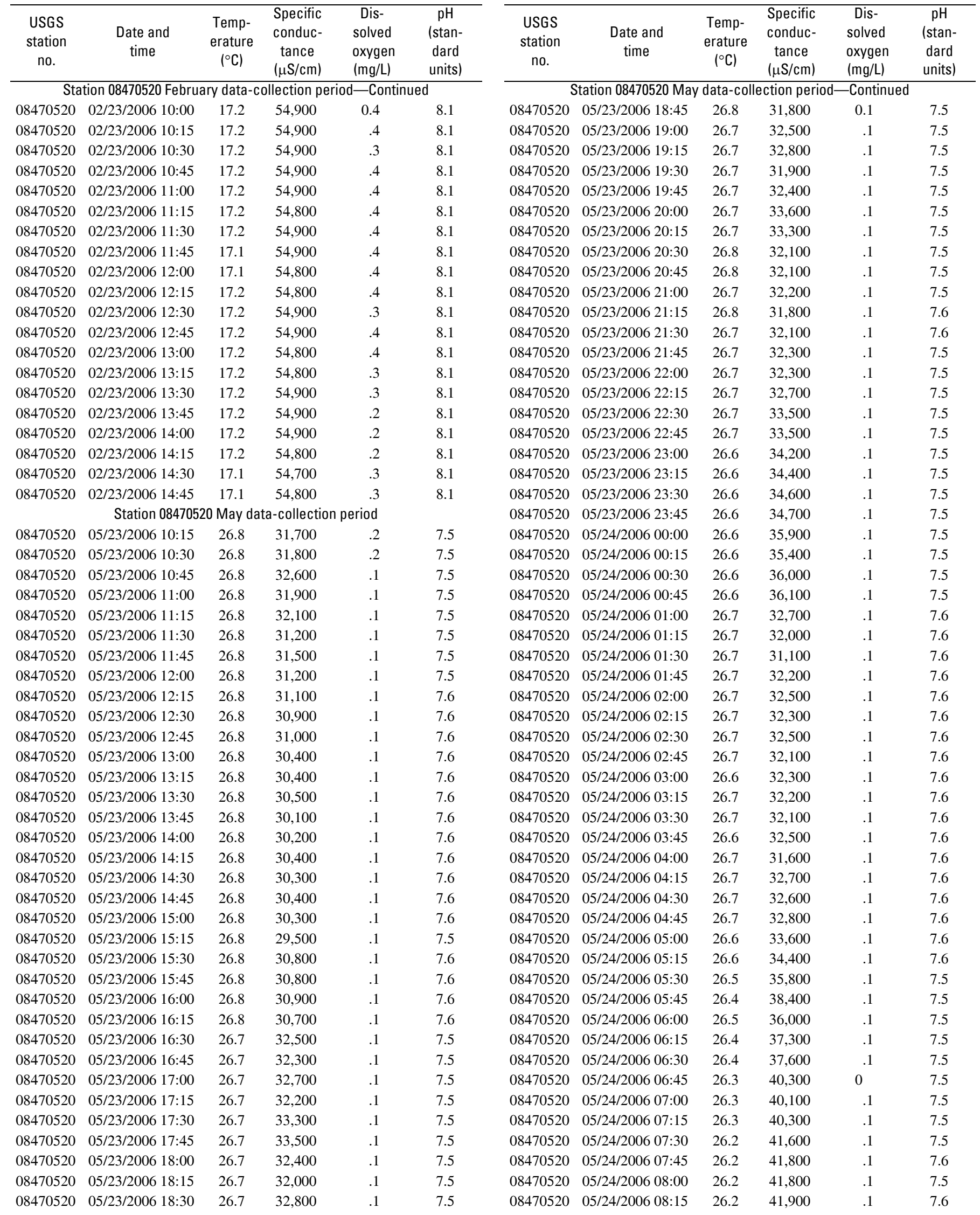


Appendix 1-1.2. Data collected at near-bottom depth of the Arroyo Colorado near Rio Hondo, Texas, using multiparameter continuous water-quality monitors-Continued.

\begin{tabular}{cccccc}
\hline $\begin{array}{c}\text { USGS } \\
\text { station } \\
\text { no. }\end{array}$ & $\begin{array}{c}\text { Date and } \\
\text { time }\end{array}$ & $\begin{array}{c}\text { Temp- } \\
\text { erature } \\
\left({ }^{\circ} \mathrm{C}\right)\end{array}$ & $\begin{array}{c}\text { Specific } \\
\text { conduc- } \\
\text { tance } \\
(\mu S / \mathrm{cm})\end{array}$ & $\begin{array}{c}\text { Dis- } \\
\text { solved } \\
\text { oxygen } \\
(\mathrm{mg} / \mathrm{L})\end{array}$ & $\begin{array}{c}\text { pH } \\
\text { (stan- } \\
\text { dard } \\
\text { units) }\end{array}$ \\
\hline \multicolumn{5}{c}{ Station 08470520 May data-collection period-Continued } \\
08470520 & $05 / 24 / 200608: 30$ & 26.2 & 41,700 & 0.1 & 7.6 \\
08470520 & $05 / 24 / 200608: 45$ & 26.2 & 41,700 & .1 & 7.6 \\
08470520 & $05 / 24 / 200609: 00$ & 26.3 & 40,500 & .1 & 7.6 \\
08470520 & $05 / 24 / 200609: 15$ & 27.0 & 27,900 & 1.8 & 7.8 \\
08470520 & $05 / 24 / 200609: 30$ & 26.9 & 29,400 & 1.2 & 7.8 \\
08470520 & $05 / 24 / 200609: 45$ & 26.8 & 29,500 & .6 & 7.8 \\
08470520 & $05 / 24 / 200610: 00$ & 26.7 & 30,800 & .1 & 7.8 \\
08470520 & $05 / 24 / 200610: 15$ & 27.0 & 27,700 & .5 & 7.8 \\
08470520 & $05 / 24 / 200610: 30$ & 26.9 & 28,300 & .3 & 7.8 \\
08470520 & $05 / 24 / 200610: 45$ & 26.8 & 29,600 & .1 & 7.8 \\
08470520 & $05 / 24 / 200611: 00$ & 26.9 & 29,200 & .1 & 7.8
\end{tabular}

\begin{tabular}{cccccc}
\hline $\begin{array}{c}\text { USGS } \\
\text { station } \\
\text { no. }\end{array}$ & $\begin{array}{c}\text { Date and } \\
\text { time }\end{array}$ & $\begin{array}{c}\text { Temp- } \\
\text { erature } \\
\left({ }^{\circ} \mathrm{C}\right)\end{array}$ & $\begin{array}{c}\text { Specific } \\
\text { conduc- } \\
\text { tance } \\
(\mu \mathrm{SS} / \mathrm{cm})\end{array}$ & $\begin{array}{c}\text { Dis- } \\
\text { solved } \\
\text { oxygen } \\
(\mathrm{mg} / \mathrm{L})\end{array}$ & $\begin{array}{c}\mathrm{pH} \\
\text { (stan- } \\
\text { dard } \\
\text { units) }\end{array}$ \\
\hline \multicolumn{5}{c}{ Station 08470520 May data-collection period-Continued } \\
08470520 & $05 / 24 / 200611: 15$ & 26.8 & 30,100 & 0.1 & 7.8 \\
08470520 & $05 / 24 / 200611: 30$ & 26.9 & 28,600 & .1 & 7.7 \\
08470520 & $05 / 24 / 200611: 45$ & 26.9 & 28,400 & .1 & 7.7 \\
08470520 & $05 / 24 / 200612: 00$ & 26.9 & 28,700 & .1 & 7.7 \\
08470520 & $05 / 24 / 200612: 15$ & 26.9 & 28,400 & .1 & 7.7 \\
08470520 & $05 / 24 / 200612: 30$ & 26.9 & 28,500 & .1 & 7.7 \\
08470520 & $05 / 24 / 200612: 45$ & 26.9 & 28,500 & .1 & 7.7 \\
08470520 & $05 / 24 / 200613: 00$ & 27.0 & 27,800 & .1 & 7.7 \\
08470520 & $05 / 24 / 200613: 15$ & 27.0 & 27,000 & .1 & 7.7 \\
08470520 & $05 / 24 / 200613: 30$ & 27.1 & 26,400 & .1 & 7.7 \\
\hline
\end{tabular}

Appendix 1-1.3. Data collected at near-bottom depth of the Arroyo Colorado near Rio Hondo, Texas, using sediment traps.

$\left[\mathrm{mg} / \mathrm{m}^{2} / \mathrm{hr}\right.$, milligrams per square meter per hour; --, not sampled; values manually rounded]

\begin{tabular}{cccccc}
\hline $\begin{array}{c}\text { USGS } \\
\text { station no. }\end{array}$ & $\begin{array}{c}\text { Date and } \\
\text { time }\end{array}$ & $\begin{array}{c}\text { Total } \\
\text { nonfilterable } \\
\text { residue } \\
\left(\mathrm{mg} / \mathrm{m}^{2} / \mathrm{hr}\right)\end{array}$ & $\begin{array}{c}\text { Ignition } \\
\text { loss from } \\
\text { nonfilterable } \\
\text { residue } \\
\left(\mathrm{mg} / \mathrm{m}^{2} / \mathrm{hr}\right)\end{array}$ & $\begin{array}{c}\text { Total } \\
\text { carbon } \\
\text { suspended } \\
\text { sediment } \\
\left(\mathrm{mg} / \mathrm{m}^{2} / \mathrm{hr}\right)\end{array}$ & $\begin{array}{c}\text { Particulate } \\
\text { nitrogen } \\
\text { suspended } \\
\text { in water } \\
\left(\mathrm{mg} / \mathrm{m}^{2} / \mathrm{hr}\right)\end{array}$ \\
\hline 08470450 & $02 / 24 / 200614: 00$ & 34,690 & 5,900 & 571.0 & 30.0 \\
08470450 & $02 / 24 / 200614: 05$ & 32,610 & 5,190 & -- & -- \\
08470460 & $02 / 24 / 200613: 00$ & 6,682 & 1,170 & 59.9 & 4.0 \\
08470460 & $02 / 24 / 200613: 05$ & 8,368 & 1,810 & -- & -- \\
08470460 & $05 / 24 / 200608: 00$ & 25,050 & 4,320 & -- & -- \\
08470460 & $05 / 24 / 200608: 05$ & 23,750 & 4,530 & -- & -- \\
08470500 & $02 / 24 / 200612: 00$ & 1,521 & 266 & 30.8 & 2.0 \\
08470500 & $02 / 24 / 200612: 05$ & 1,285 & 270 & -- & - \\
08470520 & $02 / 24 / 200611: 00$ & 1,237 & 169 & 187.0 & 10.0 \\
08470520 & $02 / 24 / 200611: 05$ & 1,442 & 308 & -- & - \\
08470520 & $05 / 24 / 200607: 00$ & 2,525 & 498 & -- & - \\
08470520 & $05 / 24 / 200607: 05$ & 2,555 & 477 & -- & - \\
\hline
\end{tabular}


Appendix 1-1.4a. Data collected at near-bottom depth of the Arroyo Colorado near Rio Hondo, Texas, using environmental discretesample analysis, February 2006.

[ft, feet; FNMU, formazin nephelometric units; $\mathrm{mmHg}$, millimeters of mercury; $\mathrm{mg} / \mathrm{L}$, milligrams per liter; --, not sampled; E, estimated; PSU, practical salinity units; $\mu \mathrm{S} / \mathrm{cm}$, microsiemens per centimeter at 25 degrees Celsius; ${ }^{\circ} \mathrm{C}$, degrees Celsius; $\mathrm{CaCO}_{3}$, calcium carbonate; $\mathrm{N}$, nitrogen; $\mathrm{NH}_{4}$, ammonia; $\mathrm{NO}_{3}$, nitrate; $\mathrm{P}$, phosphorus; $\mu \mathrm{g} / \mathrm{L}$, micrograms per liter; <, less than]

\begin{tabular}{|c|c|c|c|c|c|c|c|c|}
\hline \multirow[b]{2}{*}{ Constituent } & \multicolumn{2}{|c|}{08470450} & \multicolumn{2}{|c|}{08470460} & \multicolumn{2}{|c|}{08470500} & \multicolumn{2}{|c|}{08470520} \\
\hline & $\begin{array}{c}02 / 22 / 2006 \\
17: 00\end{array}$ & $\begin{array}{l}02 / 23 / 2006 \\
11: 00\end{array}$ & $\begin{array}{c}\text { 02/22/2006 } \\
16: 00\end{array}$ & $\begin{array}{c}02 / 23 / 2006 \\
10: 00\end{array}$ & $\begin{array}{c}02 / 22 / 2006 \\
15: 00\end{array}$ & $\begin{array}{c}\text { 02/23/2006 } \\
09: 00\end{array}$ & $\begin{array}{c}02 / 22 / 2006 \\
14: 30\end{array}$ & $\begin{array}{l}02 / 23 / 2006 \\
08: 00\end{array}$ \\
\hline Turbidity (FNMU) & 210.0 & 120.0 & 22.0 & 15.0 & 8.0 & 9.4 & 6.2 & 6.4 \\
\hline Barometric pressure (mmHg) & 762 & 762 & 761 & 762 & 760 & 760 & 762 & 762 \\
\hline Dissolved oxygen (mg/L) & 8.1 & 6.7 & E. 8 & E.5 & E1.0 & E1.2 & E.7 & E. 4 \\
\hline Dissolved oxygen (percent of saturation) & 89 & 76 & E10 & E6 & E13 & E15 & E9 & E5 \\
\hline pH (standard units) & 7.7 & 7.6 & 8.0 & 8.0 & 8.1 & 8.1 & 8.1 & 8.1 \\
\hline Salinity (PSU) & 2 & 2 & 34 & 34 & 35 & 34 & 36 & 36 \\
\hline Acid neutralizing capacity $\left(\mathrm{mg} / \mathrm{L}\right.$ as $\left.\mathrm{CaCO}_{3}\right)$ & -- & -- & -- & -- & -- & -- & -- & -- \\
\hline Chloride (mg/L) & 660 & 640 & 16,000 & 19,000 & 18,000 & 19,000 & 20,000 & 16,000 \\
\hline Sulfate $(\mathrm{mg} / \mathrm{L})$ & 640 & 630 & 2,600 & 2,800 & 2,600 & 2,700 & 2,700 & 2,600 \\
\hline Residue on evaporation dried at $180{ }^{\circ} \mathrm{C}(\mathrm{mg} / \mathrm{L})$ & 2,300 & 2,400 & 33,000 & 34,000 & 33,000 & 34,000 & 36,000 & 36,000 \\
\hline Fixed nonfilterable residue (mg/L) & 74 & 79 & 14 & 10 & 10 & 7 & 8 & 5 \\
\hline Total nonfilterable residue (mg/L) & 100 & 100 & 22 & 14 & 16 & 11 & 12 & 10 \\
\hline Ignition loss from nonfilterable residue (mg/L) & 26.0 & 21.0 & 8.4 & 4.4 & 5.6 & 4.4 & 4.4 & 4.8 \\
\hline Ammonia plus organic nitrogen $(\mathrm{mg} / \mathrm{L}$ as $\mathrm{N})$ & 1.10 & .90 & .97 & 1.20 & 1.30 & .85 & .62 & .67 \\
\hline Total nitrogen $\left(\mathrm{mg} / \mathrm{L}\right.$ as $\left.\mathrm{NO}_{3}\right)$ & 27.9 & 26.6 & 5.4 & 7.4 & 9.6 & 5.4 & 4.8 & 4.2 \\
\hline Orthophosphate (mg/L) & .399 & 1.660 & .043 & .227 & .399 & .399 & .175 & .267 \\
\hline Orthophosphate (mg/L as P) & .130 & .540 & .014 & .074 & .130 & .130 & .057 & .087 \\
\hline Phosphate (mg/L) & .560 & .550 & .094 & .088 & .120 & .150 & .088 & .088 \\
\hline Phosphorus (mg/L) & E.34 & E.39 & E. 20 & E. 24 & E.24 & E. 24 & E.18 & E.16 \\
\hline $\begin{array}{l}\text { Carbon (inorganic plus organic) suspended } \\
\text { sediment }(\mathrm{mg} / \mathrm{L})\end{array}$ & 6.0 & 5.5 & 1.8 & 1.6 & 1.1 & 1.1 & 1.0 & 1.1 \\
\hline Organic carbon, filtered (mg/L) & 3.4 & E3.2 & 5.7 & E5.3 & 5.6 & E5.5 & 5.4 & E4.1 \\
\hline Organic carbon, unfiltered (mg/L) & 3.9 & 4.0 & 5.7 & 6.2 & 5.6 & 5.6 & 5.6 & 5.6 \\
\hline Biochemical oxygen demand (mg/L) & 2.9 & 2.9 & $<2.0$ & $<2.0$ & 2.2 & $<2.0$ & $<2.0$ & $<2.0$ \\
\hline Chlorophyll $a(\mu \mathrm{g} / \mathrm{L})$ & E19.0 & E18.0 & $<5.00$ & -- & $<5.00$ & $<5.00$ & $<5.00$ & $<5.00$ \\
\hline Pheophytin $a(\mu \mathrm{g} / \mathrm{L})$ & E6.00 & $<5.00$ & $<5.00$ & -- & $<5.00$ & $<5.00$ & $<5.00$ & $<5.00$ \\
\hline
\end{tabular}


Appendix 1-1.4b. Data collected at near-bottom depth of the Arroyo Colorado near Rio Hondo, Texas, using environmental discretesample analysis, May 2006.

[ft, feet; FNMU, formazin nephelometric units; $\mathrm{mmHg}$, millimeters of mercury; $\mathrm{mg} / \mathrm{L}$, milligrams per liter; --, not sampled; E, estimated; PSU, practical salinity units; $\mu \mathrm{S} / \mathrm{cm}$, microsiemens per centimeter at 25 degrees Celsius; ${ }^{\circ} \mathrm{C}$, degrees Celsius; $\mathrm{CaCO}_{3}$, calcium carbonate; $\mathrm{N}$, nitrogen; $\mathrm{NH}_{4}$, ammonia; $\mathrm{NO}_{3}$, nitrate; $\mathrm{P}$, phosphorus; $\mu \mathrm{g} / \mathrm{l}$, micrograms per liter; <, less than]

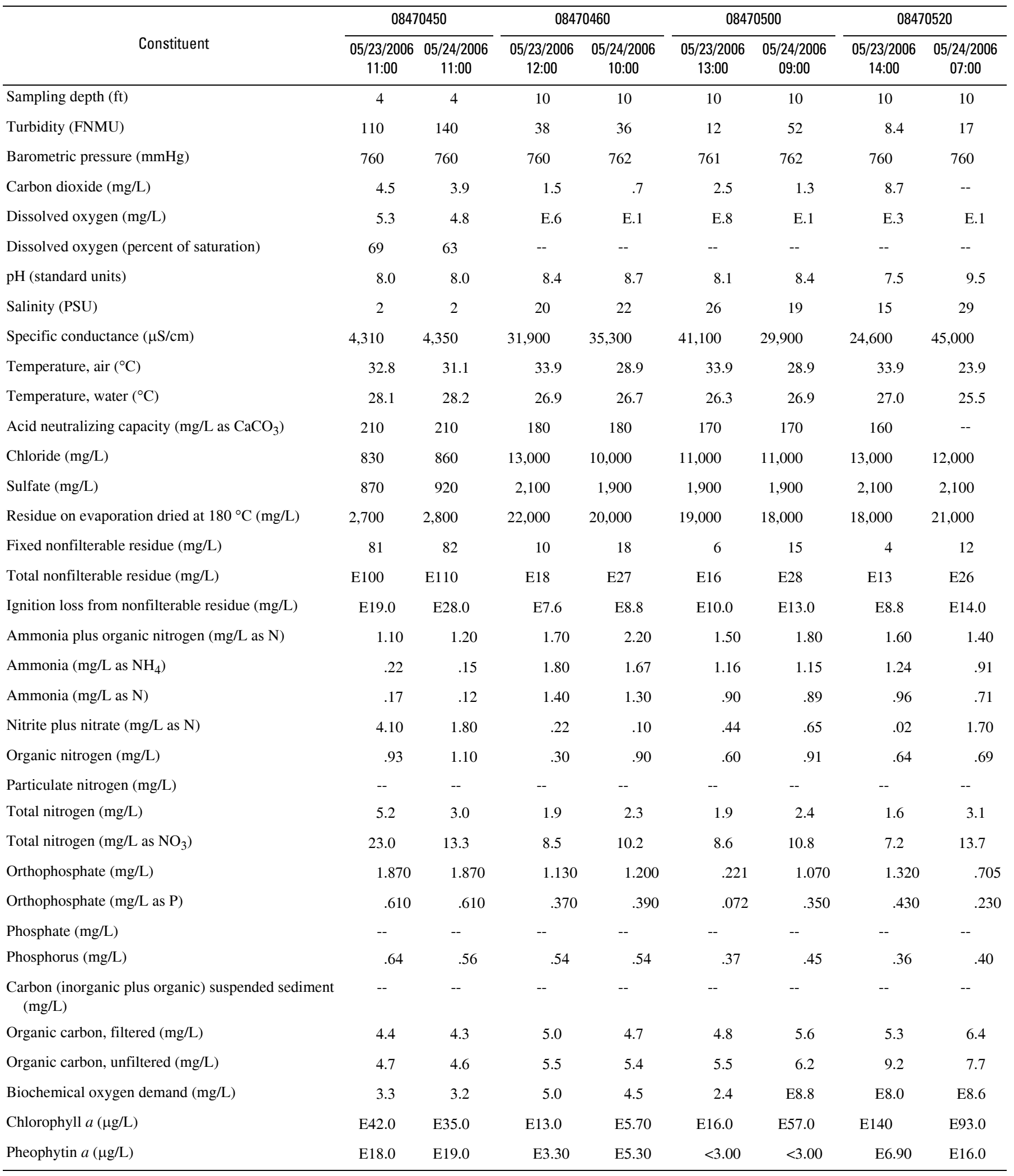




\section{Water-Quality and Ancillary Data Collected From the Arroyo Colorado Near Rio Hondo, Texas, 2006}

Appendix 1-2.1. Data collected at near-surface depth of the Arroyo Colorado near Rio Hondo, Texas, using algal productivity methods.

[ $\mathrm{mg} \mathrm{O}_{2} / \mathrm{m}^{3} /$ day, milligrams of oxygen per cubic meter per day; negative value signifies a deficit of oxygen and positive value signifies accumulation of oxygen; values manually rounded.]

\begin{tabular}{cclccc}
\hline $\begin{array}{c}\text { USGS } \\
\text { station no. }\end{array}$ & Date & $\begin{array}{c}\text { Data-collection } \\
\text { period }\end{array}$ & $\begin{array}{c}\text { Gross primary productivity } \\
\left(\mathrm{mg} \mathrm{O}_{2} / \mathrm{m}^{3} / \text { day }\right)\end{array}$ & $\begin{array}{c}\text { Net primary productivity } \\
\left(\mathrm{mg} \mathrm{O}_{2} / \mathrm{m}^{3} / \text { day }\right)\end{array}$ & $\begin{array}{c}\text { Respiration } \\
\left(\mathrm{mg} \mathrm{O}_{2} / \mathrm{m}^{3} / \text { day }\right)\end{array}$ \\
\hline 08470460 & $02 / 22 / 2006$ & February & 9,068 & 6,547 & 2,521 \\
08470460 & $05 / 23 / 2006$ & May & 16,452 & 12,875 & 3,576 \\
08470500 & $02 / 22 / 2006$ & February & 8,243 & 4,529 & 3,714 \\
08470500 & $05 / 23 / 2006$ & May & 35,660 & 31,820 & 3,840 \\
\hline
\end{tabular}

Appendix 1-2.2. Data collected at near-surface depth of the Arroyo Colorado near Rio Hondo, Texas, using multiparameter continuous water-quality monitors.

$\left[{ }^{\circ} \mathrm{C}\right.$, degrees Celsius; $\mu \mathrm{S} / \mathrm{cm}$, microsiemens per centimeter at 25 degrees Celsius; $\mathrm{mg} / \mathrm{L}$, milligrams per liter. Dissolved oxygen values less than 2.0 mg/L reported here to show resolution in a low dissolved oxygen environment, rather than set all values to $<2.0 \mathrm{mg} / \mathrm{L}$; zero values should be interpreted as $<0.1 \mathrm{mg} / \mathrm{L}]$

\begin{tabular}{|c|c|c|c|c|c|c|c|c|c|c|c|}
\hline $\begin{array}{c}\text { USGS } \\
\text { station no. }\end{array}$ & $\begin{array}{c}\text { Date } \\
\text { and time }\end{array}$ & $\begin{array}{c}\text { Temp- } \\
\text { erature } \\
\left({ }^{\circ} \mathrm{C}\right)\end{array}$ & $\begin{array}{c}\text { Specific } \\
\text { conduc- } \\
\text { tance } \\
(\mu \mathrm{S} / \mathrm{cm})\end{array}$ & $\begin{array}{l}\text { Dis- } \\
\text { solved } \\
\text { oxygen } \\
\text { (mg/L) }\end{array}$ & $\begin{array}{c}\text { pH } \\
\text { (stan- } \\
\text { dard } \\
\text { units) }\end{array}$ & $\begin{array}{c}\text { USGS } \\
\text { station no. }\end{array}$ & $\begin{array}{c}\text { Date } \\
\text { and time }\end{array}$ & $\begin{array}{c}\text { Temp- } \\
\text { erature } \\
\left({ }^{\circ} \mathrm{C}\right)\end{array}$ & $\begin{array}{c}\text { Specific } \\
\text { conduc- } \\
\text { tance } \\
(\mu \mathrm{S} / \mathrm{cm})\end{array}$ & $\begin{array}{c}\text { Dis- } \\
\text { solved } \\
\text { oxygen } \\
\text { (mg/L) }\end{array}$ & $\begin{array}{c}\mathrm{pH} \\
\text { (stan- } \\
\text { dard } \\
\text { units) }\end{array}$ \\
\hline \multicolumn{6}{|c|}{ Station 08470450 February data-collection period } & \multicolumn{6}{|c|}{ Station 08470450 February data-collection period-Continued } \\
\hline 08470450 & 02/22/2006 10:30 & 17.8 & 3,540 & 7.8 & 7.6 & 08470450 & 02/22/2006 20:15 & 19.6 & 3,750 & 7.9 & 7.7 \\
\hline 08470450 & 02/22/2006 10:45 & 17.9 & 3,540 & 7.8 & 7.6 & 08470450 & 02/22/2006 20:30 & 19.6 & 3,750 & 7.9 & 7.7 \\
\hline 08470450 & 02/22/2006 11:00 & 18.1 & 3,480 & 7.9 & 7.7 & 08470450 & $02 / 22 / 200620: 45$ & 19.7 & 3,760 & 7.8 & 7.7 \\
\hline 08470450 & $02 / 22 / 200611: 15$ & 18.1 & 3,510 & 7.9 & 7.7 & 08470450 & 02/22/2006 21:00 & 19.7 & 3,820 & 7.8 & 7.7 \\
\hline 08470450 & $02 / 22 / 200611: 30$ & 18.1 & 3,540 & 7.8 & 7.7 & 08470450 & $02 / 22 / 200621: 15$ & 19.7 & 3,760 & 7.8 & 7.7 \\
\hline 08470450 & $02 / 22 / 200611: 45$ & 18.3 & 3,480 & 7.8 & 7.7 & 08470450 & $02 / 22 / 200621: 30$ & 19.7 & 3,740 & 7.7 & 7.7 \\
\hline 08470450 & 02/22/2006 12:00 & 18.2 & 3,530 & 7.9 & 7.7 & 08470450 & $02 / 22 / 200621: 45$ & 19.7 & 3,750 & 7.7 & 7.7 \\
\hline 08470450 & $02 / 22 / 200612: 15$ & 18.3 & 3,540 & 7.9 & 7.7 & 08470450 & 02/22/2006 22:00 & 19.7 & 3,790 & 7.7 & 7.7 \\
\hline 08470450 & $02 / 22 / 200612: 30$ & 18.4 & 3,540 & 7.9 & 7.7 & 08470450 & $02 / 22 / 200622: 15$ & 19.8 & 3,820 & 7.6 & 7.7 \\
\hline 08470450 & $02 / 22 / 200612: 45$ & 18.4 & 3,540 & 8.0 & 7.7 & 08470450 & $02 / 22 / 200622: 30$ & 19.8 & 3,770 & 7.6 & 7.7 \\
\hline 08470450 & 02/22/2006 13:00 & 18.6 & 3,540 & 8.0 & 7.7 & 08470450 & $02 / 22 / 200622: 45$ & 19.8 & 3,740 & 7.6 & 7.7 \\
\hline 08470450 & $02 / 22 / 200613: 15$ & 18.6 & 3,540 & 8.0 & 7.7 & 08470450 & 02/22/2006 23:00 & 19.8 & 3,740 & 7.5 & 7.7 \\
\hline 08470450 & $02 / 22 / 200613: 30$ & 18.7 & 3,540 & 8.0 & 7.7 & 08470450 & $02 / 22 / 200623: 15$ & 19.8 & 3,740 & 7.5 & 7.7 \\
\hline 08470450 & $02 / 22 / 200613: 45$ & 18.7 & 3,560 & 8.1 & 7.7 & 08470450 & $02 / 22 / 200623: 30$ & 19.9 & 3,740 & 7.4 & 7.7 \\
\hline 08470450 & 02/22/2006 14:00 & 18.8 & 3,540 & 8.0 & 7.7 & 08470450 & $02 / 22 / 200623: 45$ & 19.9 & 3,740 & 7.4 & 7.7 \\
\hline 08470450 & $02 / 22 / 200614: 15$ & 19.0 & 3,540 & 8.1 & 7.7 & 08470450 & 02/23/2006 00:00 & 20.0 & 3,750 & 7.4 & 7.7 \\
\hline 08470450 & $02 / 22 / 200614: 30$ & 19.0 & 3,560 & 8.1 & 7.7 & 08470450 & 02/23/2006 00:15 & 20.0 & 3,750 & 7.3 & 7.7 \\
\hline 08470450 & $02 / 22 / 200614: 45$ & 19.1 & 3,560 & 8.1 & 7.7 & 08470450 & 02/23/2006 00:30 & 20.0 & 3,750 & 7.3 & 7.7 \\
\hline 08470450 & 02/22/2006 15:00 & 19.4 & 3,500 & 8.2 & 7.7 & 08470450 & 02/23/2006 00:45 & 20.1 & 3,760 & 7.2 & 7.7 \\
\hline 08470450 & $02 / 22 / 200615: 15$ & 19.5 & 3,520 & 8.1 & 7.7 & 08470450 & 02/23/2006 01:00 & 20.1 & 3,760 & 7.2 & 7.7 \\
\hline 08470450 & $02 / 22 / 200615: 30$ & 19.6 & 3,500 & 8.2 & 7.8 & 08470450 & 02/23/2006 01:15 & 20.2 & 3,760 & 7.2 & 7.7 \\
\hline 08470450 & $02 / 22 / 200615: 45$ & 19.5 & 3,540 & 8.2 & 7.7 & 08470450 & 02/23/2006 01:30 & 20.2 & 3,760 & 7.2 & 7.7 \\
\hline 08470450 & 02/22/2006 16:00 & 19.7 & 3,520 & 8.2 & 7.8 & 08470450 & 02/23/2006 01:45 & 20.3 & 3,760 & 7.1 & 7.7 \\
\hline 08470450 & $02 / 22 / 200616: 15$ & 19.7 & 3,540 & 8.2 & 7.8 & 08470450 & 02/23/2006 02:00 & 20.3 & 3,760 & 7.1 & 7.7 \\
\hline 08470450 & 02/22/2006 16:30 & 19.8 & 3,570 & 8.2 & 7.7 & 08470450 & 02/23/2006 02:15 & 20.4 & 3,760 & 7.1 & 7.7 \\
\hline 08470450 & 02/22/2006 16:45 & 19.8 & 3,550 & 8.5 & 7.8 & 08470450 & $02 / 23 / 200602: 30$ & 20.4 & 3,760 & 7.0 & 7.7 \\
\hline 08470450 & 02/22/2006 17:00 & 19.5 & 3,640 & 8.2 & 7.7 & 08470450 & 02/23/2006 02:45 & 20.4 & 3,760 & 7.0 & 7.7 \\
\hline 08470450 & 02/22/2006 17:15 & 19.5 & 3,640 & 8.2 & 7.7 & 08470450 & 02/23/2006 03:00 & 20.5 & 3,750 & 7.0 & 7.7 \\
\hline 08470450 & $02 / 22 / 200617: 30$ & 19.5 & 3,640 & 8.1 & 7.7 & 08470450 & 02/23/2006 03:15 & 20.5 & 3,750 & 7.0 & 7.7 \\
\hline 08470450 & 02/22/2006 17:45 & 19.6 & 3,650 & 8.1 & 7.7 & 08470450 & 02/23/2006 03:30 & 20.6 & 3,740 & 7.0 & 7.7 \\
\hline 08470450 & 02/22/2006 18:00 & 19.6 & 3,650 & 8.1 & 7.7 & 08470450 & 02/23/2006 03:45 & 20.6 & 3,740 & 7.0 & 7.7 \\
\hline 08470450 & 02/22/2006 18:15 & 19.6 & 3,660 & 8.1 & 7.7 & 08470450 & 02/23/2006 04:00 & 20.6 & 3,740 & 6.9 & 7.7 \\
\hline 08470450 & 02/22/2006 18:30 & 19.6 & 3,670 & 8.1 & 7.7 & 08470450 & 02/23/2006 04:15 & 20.7 & 3,730 & 6.9 & 7.7 \\
\hline 08470450 & $02 / 22 / 200618: 45$ & 19.6 & 3,710 & 8.1 & 7.7 & 08470450 & 02/23/2006 04:30 & 20.7 & 3,730 & 6.9 & 7.7 \\
\hline 08470450 & 02/22/2006 19:00 & 19.6 & 3,690 & 8.0 & 7.7 & 08470450 & 02/23/2006 04:45 & 20.7 & 3,730 & 6.9 & 7.7 \\
\hline 08470450 & 02/22/2006 19:15 & 19.6 & 3,810 & 8.0 & 7.7 & 08470450 & 02/23/2006 05:00 & 20.7 & 3,720 & 6.9 & 7.7 \\
\hline 08470450 & 02/22/2006 19:30 & 19.6 & 3,790 & 8.0 & 7.7 & 08470450 & 02/23/2006 05:15 & 20.7 & 3,720 & 6.8 & 7.7 \\
\hline 08470450 & $02 / 22 / 200619: 45$ & 19.6 & 3,800 & 8.0 & 7.7 & 08470450 & 02/23/2006 05:30 & 20.8 & 3,720 & 6.8 & 7.7 \\
\hline 08470450 & 02/22/2006 20:00 & 19.6 & 3,800 & 7.9 & 7.7 & 08470450 & 02/23/2006 05:45 & 20.8 & 3,720 & 6.8 & 7.7 \\
\hline
\end{tabular}


Appendix 1-2.2. Data collected at near-surface depth of the Arroyo Colorado near Rio Hondo, Texas, using multiparameter continuous water-quality monitors-Continued.

\begin{tabular}{|c|c|c|c|c|c|c|c|c|c|c|c|}
\hline $\begin{array}{c}\text { USGS } \\
\text { station no. }\end{array}$ & $\begin{array}{c}\text { Date } \\
\text { and time }\end{array}$ & $\begin{array}{l}\text { Temp- } \\
\text { erature } \\
\left({ }^{\circ} \mathrm{C}\right)\end{array}$ & $\begin{array}{c}\text { Specific } \\
\text { conduc- } \\
\text { tance } \\
(\mu \mathrm{S} / \mathrm{cm})\end{array}$ & $\begin{array}{l}\text { Dis- } \\
\text { solved } \\
\text { oxygen } \\
\text { (mg/L) }\end{array}$ & $\begin{array}{c}\mathrm{pH} \\
\text { (stan- } \\
\text { dard } \\
\text { units) }\end{array}$ & $\begin{array}{c}\text { USGS } \\
\text { station no. }\end{array}$ & $\begin{array}{c}\text { Date } \\
\text { and time }\end{array}$ & $\begin{array}{l}\text { Temp- } \\
\text { erature } \\
\left({ }^{\circ} \mathrm{C}\right)\end{array}$ & $\begin{array}{c}\text { Specific } \\
\text { conduc- } \\
\text { tance } \\
(\mu \mathrm{S} / \mathrm{cm})\end{array}$ & $\begin{array}{l}\text { Dis- } \\
\text { solved } \\
\text { oxygen } \\
\text { (mg/L) }\end{array}$ & $\begin{array}{l}\mathrm{pH} \\
\text { (stan- } \\
\text { dard } \\
\text { units) }\end{array}$ \\
\hline 08470450 & 02/23/2006 06:00 & 20.8 & 3,730 & 6.8 & 7.7 & 08470450 & 05/23/2006 18:00 & 29.4 & 4,320 & 6.7 & 7.8 \\
\hline 08470450 & 02/23/2006 06:15 & 20.8 & 3,730 & 6.8 & 7.7 & 08470450 & 05/23/2006 18:15 & 29.5 & 4,320 & 6.7 & 7.8 \\
\hline 08470450 & 02/23/2006 06:30 & 20.8 & 3,730 & 6.8 & 7.7 & 08470450 & 05/23/2006 18:30 & 29.6 & 4,320 & 6.8 & 7.8 \\
\hline 08470450 & 02/23/2006 07:15 & 20.9 & 3,740 & 6.7 & 7.7 & 08470450 & 05/23/2006 19:15 & 29.6 & 4,320 & 6.9 & 7.8 \\
\hline 08470450 & 02/23/2006 07:30 & 20.9 & 3,740 & 6.6 & 7.7 & 08470450 & 05/23/2006 19:30 & 29.6 & 4,320 & 6.9 & 7.8 \\
\hline 08470450 & 02/23/2006 07:45 & 20.9 & 3,750 & 6.7 & 7.7 & 08470450 & 05/23/2006 19:45 & 29.5 & 4,320 & 6.9 & 7.8 \\
\hline 08470450 & 02/23/2006 08:00 & 20.9 & 3,750 & 6.6 & 7.7 & 08470450 & 05/23/2006 20:00 & 29.5 & 4,320 & 6.9 & 7.8 \\
\hline 08470450 & 02/23/2006 08:15 & 20.9 & 3,750 & 6.6 & 7.7 & 08470450 & 05/23/2006 20:15 & 29.4 & 4,320 & 6.8 & 7.8 \\
\hline 08470450 & 02/23/2006 08:30 & 20.9 & 3,760 & 6.6 & 7.7 & 08470450 & 05/23/2006 20:30 & 29.4 & 4,320 & 6.8 & 7.8 \\
\hline 08470450 & 02/23/2006 09:45 & 21.0 & 3,780 & 6.6 & 7.7 & 08470450 & 05/23/2006 21:45 & 29.1 & 4,290 & 6.5 & 7.8 \\
\hline 08470450 & 02/23/2006 10:00 & 21.1 & 3,780 & 6.7 & 7.7 & 08470450 & 05/23/2006 22:00 & 29.0 & 4,280 & 6.5 & 7.7 \\
\hline 08470450 & 02/23/2006 10:15 & 21.0 & 3,780 & 6.6 & 7.7 & 08470450 & 05/23/2006 22:15 & 29.0 & 4,270 & 6.5 & 7.7 \\
\hline 08470450 & 02/23/2006 10:30 & 21.0 & 3,790 & 6.7 & 7.7 & 08470450 & 05/23/2006 22:30 & 29.0 & 4,270 & 6.4 & 7.7 \\
\hline 08470450 & 02/23/2006 10:45 & 21.0 & 3,790 & 6.7 & 7.7 & 08470450 & 05/23/2006 22:45 & 29.0 & 4,260 & 6.4 & 7.7 \\
\hline 08470450 & 02/23/2006 11:00 & 21.0 & 3,790 & 6.6 & 7.7 & 08470450 & 05/23/2006 23:00 & 29.0 & 4,260 & 6.4 & 7.7 \\
\hline 08470450 & $02 / 23 / 2006$ 11:15 & 21.0 & 3,790 & 6.7 & 7.7 & 08470450 & 05/23/2006 23:15 & 28.9 & 4,250 & 6.4 & 7.7 \\
\hline 08470450 & 02/23/2006 11:30 & 21.0 & 3,790 & 6.7 & 7.7 & 08470450 & 05/23/2006 23:30 & 28.9 & 4,250 & 6.3 & 7.7 \\
\hline 08470450 & 02/23/2006 11:45 & 20.9 & 3,800 & 6.8 & 7.7 & 08470450 & 05/23/2006 23:45 & 28.9 & 4,240 & 6.3 & 7.7 \\
\hline 08470450 & 02/23/2006 12:00 & 20.9 & 3,800 & 6.7 & 7.7 & 08470450 & 05/24/2006 00:00 & 28.8 & 4,240 & 6.2 & 7.7 \\
\hline 08470450 & 02/23/2006 12:15 & 20.9 & 3,800 & 6.8 & 7.7 & 08470450 & 05/24/2006 00:15 & 28.8 & 4,240 & 6.2 & 7.7 \\
\hline 08470450 & 05/23/2006 12:45 & 28.4 & 4,310 & 5.4 & 7.6 & 08470450 & 05/24/2006 02:30 & 28.5 & 4,230 & 6.0 & 7.7 \\
\hline 08470450 & 05/23/2006 13:00 & 28.4 & 4,310 & 5.5 & 7.6 & 08470450 & 05/24/2006 02:45 & 28.5 & 4,230 & 5.9 & 7.7 \\
\hline 08470450 & 05/23/2006 13:15 & 28.4 & 4,310 & 5.5 & 7.7 & 08470450 & 05/24/2006 03:00 & 28.5 & 4,230 & 5.8 & 7.7 \\
\hline 08470450 & 05/23/2006 13:30 & 28.4 & 4,310 & 5.6 & 7.7 & 08470450 & 05/24/2006 03:15 & 28.5 & 4,240 & 5.8 & 7.7 \\
\hline 08470450 & 05/23/2006 13:45 & 28.5 & 4,310 & 5.7 & 7.7 & 08470450 & 05/24/2006 03:30 & 28.5 & 4,240 & 5.7 & 7.7 \\
\hline 08470450 & 05/23/2006 14:00 & 28.5 & 4,320 & 5.7 & 7.7 & 08470450 & 05/24/2006 03:45 & 28.5 & 4,240 & 5.6 & 7.7 \\
\hline 08470450 & 05/23/2006 14:15 & 28.6 & 4,320 & 5.9 & 7.7 & 08470450 & 05/24/2006 04:00 & 28.5 & 4,250 & 5.6 & 7.7 \\
\hline 08470450 & 05/23/2006 14:30 & 28.7 & 4,320 & 6.0 & 7.7 & 08470450 & 05/24/2006 04:15 & 28.5 & 4,260 & 5.5 & 7.7 \\
\hline 08470450 & 05/23/2006 14:45 & 28.7 & 4,320 & 6.0 & 7.7 & 08470450 & 05/24/2006 04:30 & 28.4 & 4,270 & 5.4 & 7.7 \\
\hline 08470450 & 05/23/2006 15:00 & 28.8 & 4,320 & 6.1 & 7.7 & 08470450 & 05/24/2006 04:45 & 28.4 & 4,280 & 5.3 & 7.7 \\
\hline 08470450 & 05/23/2006 15:15 & 28.9 & 4,320 & 6.2 & 7.7 & 08470450 & 05/24/2006 05:00 & 28.4 & 4,290 & 5.2 & 7.7 \\
\hline 08470450 & 05/23/2006 15:30 & 28.9 & 4,320 & 6.2 & 7.7 & 08470450 & 05/24/2006 05:15 & 28.4 & 4,290 & 5.2 & 7.7 \\
\hline 08470450 & 05/23/2006 15:45 & 29.0 & 4,320 & 6.3 & 7.7 & 08470450 & 05/24/2006 05:30 & 28.4 & 4,300 & 5.1 & 7.7 \\
\hline 08470450 & 05/23/2006 16:00 & 29.1 & 4,320 & 6.4 & 7.7 & 08470450 & 05/24/2006 05:45 & 28.4 & 4,310 & 5.0 & 7.7 \\
\hline 08470450 & 05/23/2006 16:15 & 29.2 & 4,310 & 6.4 & 7.7 & 08470450 & 05/24/2006 06:00 & 28.4 & 4,320 & 5.0 & 7.7 \\
\hline 08470450 & 05/23/2006 16:30 & 29.2 & 4,310 & 6.4 & 7.7 & 08470450 & 05/24/2006 06:15 & 28.4 & 4,330 & 4.9 & 7.7 \\
\hline 08470450 & 05/23/2006 16:45 & 29.2 & 4,310 & 6.4 & 7.7 & 08470450 & 05/24/2006 06:30 & 28.4 & 4,340 & 4.9 & 7.7 \\
\hline 08470450 & 05/23/2006 17:00 & 29.2 & 4,310 & 6.4 & 7.7 & 08470450 & 05/24/2006 06:45 & 28.4 & 4,340 & 4.9 & 7.7 \\
\hline 08470450 & 05/23/2006 17:15 & 29.3 & 4,320 & 6.5 & 7.8 & 08470450 & 05/24/2006 07:00 & 28.4 & 4,350 & 4.9 & 7.7 \\
\hline 08470450 & 05/23/2006 17:30 & 29.3 & 4,320 & 6.5 & 7.8 & 08470450 & 05/24/2006 07:15 & 28.4 & 4,350 & 4.9 & 7.7 \\
\hline 08470450 & 05/23/2006 17:45 & 29.4 & 4,320 & 6.6 & 7.8 & 08470450 & 05/24/2006 07:30 & 28.3 & 4,350 & 4.9 & 7.7 \\
\hline
\end{tabular}


Appendix 1-2.2. Data collected at near-surface depth of the Arroyo Colorado near Rio Hondo, Texas, using multiparameter continuous water-quality monitors-Continued.

\begin{tabular}{|c|c|c|c|c|c|c|c|c|c|c|c|}
\hline $\begin{array}{c}\text { USGS } \\
\text { station no. }\end{array}$ & $\begin{array}{c}\text { Date } \\
\text { and time }\end{array}$ & $\begin{array}{l}\text { Temp- } \\
\text { erature } \\
\left({ }^{\circ} \mathrm{C}\right)\end{array}$ & $\begin{array}{c}\text { Specific } \\
\text { conduc- } \\
\text { tance } \\
(\mu \mathrm{S} / \mathrm{cm})\end{array}$ & $\begin{array}{l}\text { Dis- } \\
\text { solved } \\
\text { oxygen } \\
\text { (mg/L) }\end{array}$ & $\begin{array}{l}\mathrm{pH} \\
\text { (stan- } \\
\text { dard } \\
\text { units) }\end{array}$ & $\begin{array}{c}\text { USGS } \\
\text { station no. }\end{array}$ & $\begin{array}{c}\text { Date } \\
\text { and time }\end{array}$ & $\begin{array}{c}\text { Temp- } \\
\text { erature } \\
\left({ }^{\circ} \mathrm{C}\right)\end{array}$ & $\begin{array}{c}\text { Specific } \\
\text { conduc- } \\
\text { tance } \\
(\mu \mathrm{S} / \mathrm{cm})\end{array}$ & $\begin{array}{c}\begin{array}{c}\text { Dis- } \\
\text { solved } \\
\text { oxygen } \\
\text { (mg/L) }\end{array}\end{array}$ & $\begin{array}{c}\mathrm{pH} \\
\text { (stan- } \\
\text { dard } \\
\text { units) }\end{array}$ \\
\hline \multicolumn{6}{|c|}{ Station 08470450 May data-collection period-Continued } & \multicolumn{6}{|c|}{ Station 08470460 February data-collection period-Continued } \\
\hline 08470450 & 05/24/2006 07:45 & 28.3 & 4,360 & 4.9 & 7.7 & 08470460 & 02/22/2006 18:00 & 18.7 & 22,600 & 6.2 & 7.8 \\
\hline 08470450 & 05/24/2006 08:00 & 28.3 & 4,360 & 4.9 & 7.7 & 08470460 & 02/22/2006 18:15 & 18.6 & 22,600 & 6.0 & 7.7 \\
\hline 08470450 & 05/24/2006 08:15 & 28.2 & 4,360 & 4.8 & 7.7 & 08470460 & $02 / 22 / 200618: 30$ & 18.8 & 21,900 & 5.8 & 7.7 \\
\hline 08470450 & 05/24/2006 08:30 & 28.2 & 4,370 & 4.7 & 7.7 & 08470460 & 02/22/2006 18:45 & 18.9 & 20,300 & 6.2 & 7.8 \\
\hline 08470450 & 05/24/2006 08:45 & 28.2 & 4,370 & 4.7 & 7.7 & 08470460 & 02/22/2006 19:00 & 19.0 & 18,900 & 6.3 & 7.8 \\
\hline 08470450 & 05/24/2006 09:00 & 28.2 & 4,380 & 4.8 & 7.7 & 08470460 & 02/22/2006 19:15 & 19.0 & 18,700 & 6.2 & 7.7 \\
\hline 08470450 & 05/24/2006 09:15 & 28.1 & 4,380 & 4.8 & 7.7 & 08470460 & 02/22/2006 19:30 & 19.0 & 19,000 & 6.1 & 7.7 \\
\hline 08470450 & 05/24/2006 09:30 & 28.1 & 4,390 & 4.8 & 7.7 & 08470460 & 02/22/2006 19:45 & 19.0 & 19,400 & 6.2 & 7.7 \\
\hline 08470450 & 05/24/2006 09:45 & 28.1 & 4,390 & 4.8 & 7.7 & 08470460 & 02/22/2006 20:00 & 19.0 & 18,500 & 6.2 & 7.7 \\
\hline 08470450 & 05/24/2006 10:00 & 28.1 & 4,390 & 4.8 & 7.7 & 08470460 & 02/22/2006 20:15 & 19.1 & 17,700 & 6.2 & 7.7 \\
\hline 08470450 & 05/24/2006 10:15 & 28.1 & 4,390 & 4.8 & 7.7 & 08470460 & 02/22/2006 20:30 & 19.1 & 16,900 & 6.3 & 7.7 \\
\hline 08470450 & 05/24/2006 10:30 & 28.1 & 4,380 & 4.8 & 7.7 & 08470460 & 02/22/2006 20:45 & 19.1 & 16,100 & 6.3 & 7.7 \\
\hline 08470450 & 05/24/2006 10:45 & 28.1 & 4,380 & 4.8 & 7.7 & 08470460 & 02/22/2006 21:00 & 19.2 & 15,000 & 6.5 & 7.7 \\
\hline 08470450 & 05/24/2006 11:00 & 28.1 & 4,370 & 4.9 & 7.7 & 08470460 & 02/22/2006 21:15 & 19.3 & 13,600 & 6.6 & 7.7 \\
\hline 08470450 & 05/24/2006 11:15 & 28.1 & 4,370 & 5.0 & 7.7 & 08470460 & 02/22/2006 21:30 & 19.3 & 12,900 & 6.6 & 7.7 \\
\hline 08470450 & 05/24/2006 11:30 & 28.2 & 4,360 & 5.0 & 7.7 & 08470460 & 02/22/2006 21:45 & 19.4 & 12,900 & 6.6 & 7.7 \\
\hline 08470450 & 05/24/2006 11:45 & 28.2 & 4,360 & 5.1 & 7.7 & 08470460 & 02/22/2006 22:00 & 19.4 & 11,900 & 6.7 & 7.7 \\
\hline 08470450 & 05/24/2006 12:00 & 28.4 & 4,360 & 5.2 & 7.7 & 08470460 & 02/22/2006 22:15 & 19.4 & 11,200 & 6.7 & 7.7 \\
\hline 08470450 & 05/24/2006 12:15 & 28.4 & 4,350 & 5.2 & 7.7 & 08470460 & $02 / 22 / 200622: 30$ & 19.4 & 10,900 & 6.8 & 7.7 \\
\hline 08470450 & 05/24/2006 12:30 & 28.5 & 4,340 & 5.3 & 7.7 & 08470460 & 02/22/2006 22:45 & 19.4 & 11,200 & 6.8 & 7.7 \\
\hline 08470450 & 05/24/2006 12:45 & 28.5 & 4,340 & 5.4 & 7.7 & 08470460 & 02/22/2006 23:00 & 19.5 & 10,100 & 6.9 & 7.7 \\
\hline \multicolumn{6}{|c|}{ Station 08470460 February data-collection period } & 08470460 & 02/22/2006 23:15 & 19.5 & 10,200 & 6.8 & 7.7 \\
\hline 08470460 & 02/22/2006 09:45 & 17.6 & 4,380 & 8.2 & 7.6 & 08470460 & 02/22/2006 23:30 & 19.5 & 10,700 & 6.7 & 7.7 \\
\hline 08470460 & 02/22/2006 10:00 & 17.7 & 4,400 & 8.2 & 7.7 & 08470460 & 02/22/2006 23:45 & 19.6 & 9,660 & 6.8 & 7.7 \\
\hline 08470460 & 02/22/2006 10:15 & 17.8 & 4,440 & 8.2 & 7.7 & 08470460 & 02/23/2006 00:00 & 19.7 & 8,720 & 6.8 & 7.7 \\
\hline 08470460 & 02/22/2006 10:30 & 17.9 & 4,590 & 8.1 & 7.7 & 08470460 & 02/23/2006 00:15 & 19.7 & 8,560 & 6.9 & 7.7 \\
\hline 08470460 & 02/22/2006 10:45 & 18.0 & 4,680 & 8.1 & 7.7 & 08470460 & 02/23/2006 00:30 & 19.7 & 8,740 & 6.9 & 7.7 \\
\hline 08470460 & 02/22/2006 11:00 & 18.1 & 4,850 & 8.2 & 7.7 & 08470460 & 02/23/2006 00:45 & 19.7 & 8,410 & 6.9 & 7.7 \\
\hline 08470460 & 02/22/2006 11:15 & 18.2 & 5,080 & 8.1 & 7.7 & 08470460 & 02/23/2006 01:00 & 19.8 & 8,160 & 6.8 & 7.7 \\
\hline 08470460 & 02/22/2006 11:30 & 18.3 & 5,310 & 8.2 & 7.7 & 08470460 & 02/23/2006 01:15 & 19.8 & 7,840 & 6.8 & 7.7 \\
\hline 08470460 & 02/22/2006 11:45 & 18.4 & 5,430 & 8.2 & 7.7 & 08470460 & 02/23/2006 01:30 & 19.8 & 7,780 & 6.9 & 7.7 \\
\hline 08470460 & 02/22/2006 12:00 & 18.4 & 5,860 & 8.2 & 7.7 & 08470460 & 02/23/2006 01:45 & 19.8 & 7,960 & 6.8 & 7.7 \\
\hline 08470460 & 02/22/2006 12:15 & 18.6 & 6,240 & 8.2 & 7.7 & 08470460 & 02/23/2006 02:00 & 19.8 & 7,430 & 6.8 & 7.7 \\
\hline 08470460 & 02/22/2006 12:30 & 18.7 & 6,930 & 8.1 & 7.7 & 08470460 & 02/23/2006 02:15 & 19.9 & 7,280 & 6.9 & 7.7 \\
\hline 08470460 & 02/22/2006 12:45 & 19.0 & 7,690 & 8.0 & 7.7 & 08470460 & 02/23/2006 02:30 & 19.9 & 6,990 & 6.8 & 7.7 \\
\hline 08470460 & 02/22/2006 13:00 & 18.6 & 11,500 & 7.6 & 7.7 & 08470460 & 02/23/2006 02:45 & 20.0 & 6,750 & 6.8 & 7.7 \\
\hline 08470460 & 02/22/2006 13:15 & 18.6 & 13,000 & 7.3 & 7.7 & 08470460 & 02/23/2006 03:00 & 20.0 & 6,620 & 6.8 & 7.7 \\
\hline 08470460 & 02/22/2006 13:30 & 18.6 & 16,000 & 7.0 & 7.6 & 08470460 & 02/23/2006 03:15 & 20.0 & 6,600 & 6.8 & 7.7 \\
\hline 08470460 & 02/22/2006 13:45 & 18.1 & 22,000 & 6.1 & 7.6 & 08470460 & 02/23/2006 03:30 & 20.1 & 6,520 & 6.8 & 7.7 \\
\hline 08470460 & 02/22/2006 14:00 & 18.1 & 22,900 & 5.7 & 7.6 & 08470460 & 02/23/2006 03:45 & 20.1 & 6,390 & 6.7 & 7.7 \\
\hline 08470460 & 02/22/2006 14:15 & 18.6 & 21,800 & 6.2 & 7.7 & 08470460 & 02/23/2006 04:00 & 20.1 & 6,300 & 6.7 & 7.7 \\
\hline 08470460 & $02 / 22 / 200614: 30$ & 18.4 & 24,100 & 5.3 & 7.7 & 08470460 & 02/23/2006 04:15 & 20.2 & 6,210 & 6.7 & 7.7 \\
\hline 08470460 & 02/22/2006 14:45 & 18.1 & 25,400 & 5.2 & 7.7 & 08470460 & 02/23/2006 04:30 & 20.2 & 6,050 & 6.7 & 7.7 \\
\hline 08470460 & 02/22/2006 15:00 & 18.2 & 29,000 & 5.3 & 7.7 & 08470460 & 02/23/2006 04:45 & 20.2 & 5,880 & 6.7 & 7.7 \\
\hline 08470460 & 02/22/2006 15:15 & 18.7 & 26,500 & 5.7 & 7.7 & 08470460 & 02/23/2006 05:00 & 20.2 & 5,790 & 6.6 & 7.7 \\
\hline 08470460 & 02/22/2006 15:30 & 18.2 & 28,600 & 5.5 & 7.7 & 08470460 & 02/23/2006 05:15 & 20.2 & 5,750 & 6.7 & 7.7 \\
\hline 08470460 & 02/22/2006 15:45 & 19.2 & 25,200 & 5.8 & 7.7 & 08470460 & 02/23/2006 05:30 & 20.3 & 5,660 & 6.7 & 7.7 \\
\hline 08470460 & 02/22/2006 16:00 & 18.4 & 27,600 & 5.8 & 7.7 & 08470460 & 02/23/2006 05:45 & 20.3 & 5,480 & 6.7 & 7.7 \\
\hline 08470460 & 02/22/2006 16:15 & 19.0 & 26,300 & 5.8 & 7.7 & 08470460 & 02/23/2006 06:00 & 20.4 & 5,390 & 6.6 & 7.7 \\
\hline 08470460 & 02/22/2006 16:30 & 18.6 & 27,300 & 5.1 & 7.7 & 08470460 & 02/23/2006 06:15 & 20.4 & 5,150 & 6.7 & 7.7 \\
\hline 08470460 & 02/22/2006 16:45 & 19.2 & 25,400 & 5.9 & 7.7 & 08470460 & 02/23/2006 06:30 & 20.4 & 5,290 & 6.6 & 7.7 \\
\hline 08470460 & 02/22/2006 17:00 & 19.3 & 25,500 & 6.0 & 7.8 & 08470460 & 02/23/2006 06:45 & 20.5 & 5,190 & 6.6 & 7.7 \\
\hline 08470460 & 02/22/2006 17:15 & 18.6 & 24,200 & 6.1 & 7.7 & 08470460 & 02/23/2006 07:00 & 20.5 & 4,990 & 6.6 & 7.7 \\
\hline 08470460 & 02/22/2006 17:30 & 19.1 & 21,800 & 6.2 & 7.8 & 08470460 & 02/23/2006 07:15 & 20.6 & 4,780 & 6.6 & 7.7 \\
\hline 08470460 & 02/22/2006 17:45 & 18.6 & 23,900 & 5.6 & 7.7 & 08470460 & 02/23/2006 07:30 & 20.6 & 4,640 & 6.7 & 7.7 \\
\hline
\end{tabular}


Appendix 1-2.2. Data collected at near-surface depth of the Arroyo Colorado near Rio Hondo, Texas, using multiparameter continuous water-quality monitors-Continued.

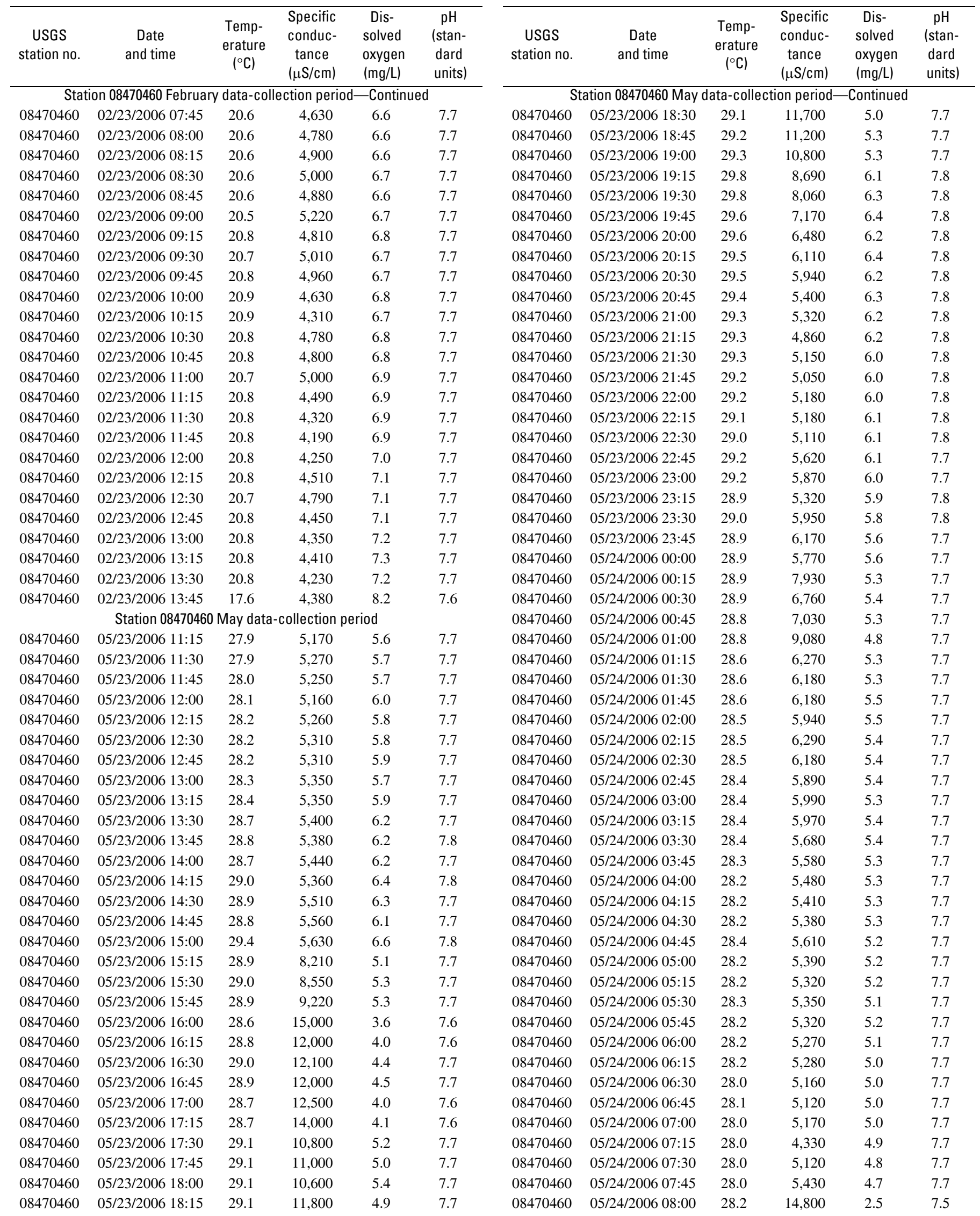


Appendix 1-2.2. Data collected at near-surface depth of the Arroyo Colorado near Rio Hondo, Texas, using multiparameter continuous water-quality monitors-Continued.

\begin{tabular}{|c|c|c|c|c|c|c|c|c|c|c|c|}
\hline $\begin{array}{c}\text { USGS } \\
\text { station no. }\end{array}$ & $\begin{array}{c}\text { Date } \\
\text { and time }\end{array}$ & $\begin{array}{l}\text { Temp- } \\
\text { erature } \\
\left({ }^{\circ} \mathrm{C}\right)\end{array}$ & $\begin{array}{c}\text { Specific } \\
\text { conduc- } \\
\text { tance } \\
(\mu \mathrm{S} / \mathrm{cm})\end{array}$ & $\begin{array}{c}\begin{array}{c}\text { Dis- } \\
\text { solved } \\
\text { oxygen } \\
\text { (mg/L) }\end{array}\end{array}$ & $\begin{array}{c}\mathrm{pH} \\
\text { (stan- } \\
\text { dard } \\
\text { units) }\end{array}$ & $\begin{array}{c}\text { USGS } \\
\text { station no. }\end{array}$ & $\begin{array}{c}\text { Date } \\
\text { and time }\end{array}$ & $\begin{array}{l}\text { Temp- } \\
\text { erature } \\
\left({ }^{\circ} \mathrm{C}\right)\end{array}$ & $\begin{array}{c}\text { Specific } \\
\text { conduc- } \\
\text { tance } \\
(\mu \mathrm{S} / \mathrm{cm})\end{array}$ & $\begin{array}{c}\begin{array}{c}\text { Dis- } \\
\text { solved } \\
\text { oxygen } \\
\text { (mg/L) }\end{array}\end{array}$ & $\begin{array}{c}\mathrm{pH} \\
\text { (stan- } \\
\text { dard } \\
\text { units) }\end{array}$ \\
\hline 08470460 & 05/24/2006 08:15 & 28.0 & 7,960 & 4.1 & 7.6 & 08470500 & 02/22/2006 19:40 & 19.4 & 18,800 & 5.7 & 7.8 \\
\hline 08470460 & 05/24/2006 08:30 & 28.1 & 7,220 & 4.1 & 7.6 & 08470500 & 02/22/2006 19:55 & 19.6 & 19,000 & 5.7 & 7.8 \\
\hline 08470460 & 05/24/2006 08:45 & 27.9 & 5,850 & 4.5 & 7.7 & 08470500 & 02/22/2006 20:10 & 19.4 & 19,000 & 5.6 & 7.8 \\
\hline 08470460 & 05/24/2006 09:30 & 28.0 & 5,920 & 4.4 & 7.6 & 08470500 & 02/22/2006 20:55 & 19.4 & 19,200 & 5.7 & 7.8 \\
\hline 08470460 & 05/24/2006 09:45 & 28.0 & 5,860 & 4.3 & 7.6 & 08470500 & 02/22/2006 21:10 & 19.4 & 19,200 & 5.5 & 7.8 \\
\hline 08470460 & 05/24/2006 10:00 & 28.0 & 5,880 & 4.5 & 7.7 & 08470500 & 02/22/2006 21:25 & 19.4 & 18,900 & 5.5 & 7.8 \\
\hline 08470460 & 05/24/2006 10:15 & 28.0 & 5,950 & 4.3 & 7.6 & 08470500 & 02/22/2006 21:40 & 19.6 & 19,000 & 5.9 & 7.8 \\
\hline 08470460 & 05/24/2006 10:30 & 28.1 & 6,540 & 4.4 & 7.6 & 08470500 & 02/22/2006 21:55 & 19.6 & 19,300 & 5.8 & 7.8 \\
\hline 08470460 & 05/24/2006 10:45 & 28.2 & 6,380 & 4.8 & 7.7 & 08470500 & 02/22/2006 22:10 & 19.3 & 19,200 & 5.3 & 7.8 \\
\hline 08470460 & 05/24/2006 12:00 & 28.3 & 6,990 & 4.7 & 7.7 & 08470500 & 02/22/2006 23:25 & 19.5 & 18,200 & 5.8 & 7.8 \\
\hline 08470460 & 05/24/2006 12:15 & 28.4 & 6,680 & 5.2 & 7.7 & 08470500 & 02/22/2006 23:40 & 19.6 & 18,300 & 5.8 & 7.8 \\
\hline 08470460 & 05/24/2006 12:30 & 28.4 & 6,820 & 4.9 & 7.7 & 08470500 & 02/22/2006 23:55 & 19.5 & 18,300 & 5.8 & 7.8 \\
\hline 08470460 & 05/24/2006 12:45 & 28.4 & 6,750 & 5.2 & 7.7 & 08470500 & 02/23/2006 00:10 & 19.5 & 18,000 & 6.0 & 7.8 \\
\hline 08470460 & 05/24/2006 13:00 & 28.5 & 6,580 & 5.5 & 7.7 & 08470500 & 02/23/2006 00:25 & 19.5 & 17,700 & 6.0 & 7.8 \\
\hline \multicolumn{6}{|c|}{ Station 08470500 February data-collection period } & 08470500 & 02/23/2006 00:40 & 19.5 & 17,300 & 5.9 & 7.8 \\
\hline 08470500 & 02/22/2006 11:10 & 17.5 & 8,500 & 7.8 & 7.7 & 08470500 & 02/23/2006 00:55 & 19.6 & 17,200 & 6.0 & 7.8 \\
\hline 08470500 & $02 / 22 / 200611: 25$ & 17.6 & 7,870 & 7.6 & 7.8 & 08470500 & 02/23/2006 01:10 & 19.6 & 16,800 & 6.1 & 7.8 \\
\hline 08470500 & 02/22/2006 11:40 & 17.6 & 10,200 & 7.3 & 7.8 & 08470500 & 02/23/2006 01:25 & 19.6 & 16,600 & 6.1 & 7.8 \\
\hline 08470500 & $02 / 22 / 200611: 55$ & 17.7 & 10,200 & 7.2 & 7.8 & 08470500 & 02/23/2006 01:40 & 19.6 & 16,600 & 6.1 & 7.8 \\
\hline 08470500 & 02/22/2006 12:10 & 17.8 & 11,900 & 7.0 & 7.8 & 08470500 & 02/23/2006 01:55 & 19.6 & 16,500 & 6.1 & 7.8 \\
\hline 08470500 & $02 / 22 / 200614: 25$ & 18.8 & 13,900 & 6.7 & 7.9 & 08470500 & 02/23/2006 04:10 & 19.5 & 15,400 & 6.3 & 7.8 \\
\hline 08470500 & 02/22/2006 14:40 & 18.8 & 14,000 & 6.5 & 7.8 & 08470500 & 02/23/2006 04:25 & 19.6 & 15,300 & 6.3 & 7.8 \\
\hline 08470500 & 02/22/2006 14:55 & 18.9 & 15,500 & 6.6 & 7.9 & 08470500 & 02/23/2006 04:40 & 19.6 & 15,200 & 6.3 & 7.8 \\
\hline 08470500 & $02 / 22 / 2006$ 15:10 & 19.1 & 15,100 & 6.6 & 7.9 & 08470500 & 02/23/2006 04:55 & 19.5 & 14,400 & 6.2 & 7.8 \\
\hline 08470500 & $02 / 22 / 2006$ 15:25 & 19.1 & 16,200 & 6.4 & 7.9 & 08470500 & 02/23/2006 05:10 & 19.5 & 14,400 & 6.1 & 7.8 \\
\hline 08470500 & $02 / 22 / 2006$ 15:40 & 19.2 & 15,400 & 6.6 & 7.9 & 08470500 & 02/23/2006 05:25 & 19.5 & 13,700 & 5.9 & 7.8 \\
\hline 08470500 & $02 / 22 / 2006$ 15:55 & 19.1 & 15,400 & 6.5 & 7.9 & 08470500 & 02/23/2006 05:40 & 19.5 & 13,600 & 6.0 & 7.8 \\
\hline 08470500 & 02/22/2006 16:10 & 19.3 & 16,300 & 6.5 & 7.9 & 08470500 & 02/23/2006 05:55 & 19.5 & 12,700 & 6.0 & 7.8 \\
\hline 08470500 & $02 / 22 / 200616: 25$ & 19.2 & 17,000 & 6.3 & 7.8 & 08470500 & 02/23/2006 06:10 & 19.5 & 12,600 & 6.0 & 7.8 \\
\hline 08470500 & 02/22/2006 16:40 & 19.4 & 15,200 & 6.6 & 7.9 & 08470500 & 02/23/2006 06:25 & 19.5 & 11,300 & 6.0 & 7.8 \\
\hline 08470500 & 02/22/2006 16:55 & 19.5 & 14,100 & 6.5 & 7.9 & 08470500 & 02/23/2006 06:40 & 19.5 & 12,300 & 6.0 & 7.8 \\
\hline 08470500 & 02/22/2006 17:10 & 19.5 & 15,000 & 6.5 & 7.9 & 08470500 & 02/23/2006 06:55 & 19.5 & 11,700 & 6.0 & 7.8 \\
\hline 08470500 & $02 / 22 / 200617: 25$ & 19.5 & 16,200 & 6.4 & 7.8 & 08470500 & 02/23/2006 07:10 & 19.5 & 12,300 & 6.0 & 7.8 \\
\hline 08470500 & $02 / 22 / 2006$ 17:40 & 19.5 & 16,600 & 6.3 & 7.8 & 08470500 & 02/23/2006 07:25 & 19.4 & 12,500 & 5.9 & 7.8 \\
\hline 08470500 & $02 / 22 / 2006$ 17:55 & 19.5 & 16,400 & 6.3 & 7.8 & 08470500 & 02/23/2006 07:40 & 19.6 & 9,620 & 6.0 & 7.8 \\
\hline 08470500 & 02/22/2006 18:10 & 19.5 & 17,800 & 6.2 & 7.8 & 08470500 & 02/23/2006 07:55 & 19.6 & 10,500 & 6.0 & 7.8 \\
\hline 08470500 & $02 / 22 / 200618: 25$ & 19.5 & 17,700 & 6.0 & 7.8 & 08470500 & 02/23/2006 08:10 & 19.7 & 9,790 & 6.0 & 7.8 \\
\hline 08470500 & $02 / 22 / 2006$ 18:40 & 19.6 & 18,000 & 6.0 & 7.8 & 08470500 & 02/23/2006 08:25 & 19.7 & 9,150 & 6.1 & 7.8 \\
\hline 08470500 & 02/22/2006 18:55 & 19.5 & 18,100 & 6.0 & 7.8 & 08470500 & 02/23/2006 08:40 & 19.7 & 10,200 & 6.0 & 7.8 \\
\hline 08470500 & 02/22/2006 19:10 & 19.5 & 18,100 & 6.0 & 7.8 & 08470500 & 02/23/2006 08:55 & 19.7 & 9,790 & 6.1 & 7.8 \\
\hline 08470500 & $02 / 22 / 200619: 25$ & 19.5 & 18,300 & 5.9 & 7.8 & 08470500 & 02/23/2006 09:10 & 19.7 & 10,200 & 6.1 & 7.8 \\
\hline
\end{tabular}


Appendix 1-2.2. Data collected at near-surface depth of the Arroyo Colorado near Rio Hondo, Texas, using multiparameter continuous water-quality monitors-Continued.

\begin{tabular}{|c|c|c|c|c|c|c|c|c|c|c|c|}
\hline $\begin{array}{c}\text { USGS } \\
\text { station no. }\end{array}$ & $\begin{array}{c}\text { Date } \\
\text { and time }\end{array}$ & $\begin{array}{l}\text { Temp- } \\
\text { erature } \\
\left({ }^{\circ} \mathrm{C}\right)\end{array}$ & $\begin{array}{l}\text { Specific } \\
\text { conduc- } \\
\text { tance } \\
(\mu \mathrm{S} / \mathrm{cm})\end{array}$ & $\begin{array}{l}\text { Dis- } \\
\text { solved } \\
\text { oxygen } \\
\text { (mg/L) }\end{array}$ & $\begin{array}{l}\mathrm{pH} \\
\text { (stan- } \\
\text { dard } \\
\text { units) }\end{array}$ & $\begin{array}{c}\text { USGS } \\
\text { station no. }\end{array}$ & $\begin{array}{c}\text { Date } \\
\text { and time }\end{array}$ & $\begin{array}{l}\text { Temp- } \\
\text { erature } \\
\left({ }^{\circ} \mathrm{C}\right)\end{array}$ & $\begin{array}{c}\text { Specific } \\
\text { conduc- } \\
\text { tance } \\
(\mu \mathrm{S} / \mathrm{cm})\end{array}$ & $\begin{array}{l}\text { Dis- } \\
\text { solved } \\
\text { oxygen } \\
\text { (mg/L) }\end{array}$ & $\begin{array}{l}\mathrm{pH} \\
\text { (stan- } \\
\text { dard } \\
\text { units) }\end{array}$ \\
\hline 08470500 & 02/23/2006 09:25 & 19.8 & 9,340 & 6.2 & 7.8 & 08470500 & 05/23/2006 19:00 & 30.2 & 6,810 & 9.0 & 8.0 \\
\hline 08470500 & 02/23/2006 09:40 & 19.8 & 10,500 & 6.2 & 7.8 & 08470500 & 05/23/2006 19:15 & 30.3 & 6,830 & 9.4 & 8.0 \\
\hline 08470500 & 02/23/2006 09:55 & 19.8 & 8,750 & 6.4 & 7.8 & 08470500 & 05/23/2006 19:30 & 30.3 & 6,680 & 9.6 & 8.0 \\
\hline 08470500 & 02/23/2006 10:40 & 19.9 & 8,800 & 6.7 & 7.8 & 08470500 & 05/23/2006 20:15 & 29.9 & 6,880 & 9.4 & 8.0 \\
\hline 08470500 & 02/23/2006 10:55 & 19.8 & 9,320 & 6.6 & 7.8 & 08470500 & 05/23/2006 20:30 & 29.9 & 6,870 & 9.3 & 8.0 \\
\hline 08470500 & 02/23/2006 11:10 & 19.7 & 10,100 & 6.6 & 7.8 & 08470500 & 05/23/2006 20:45 & 29.8 & 6,880 & 9.2 & 8.0 \\
\hline 08470500 & $02 / 23 / 200611: 25$ & 19.7 & 10,800 & 6.7 & 7.8 & 08470500 & 05/23/2006 21:00 & 29.6 & 6,860 & 9.2 & 8.0 \\
\hline 08470500 & 02/23/2006 11:40 & 19.7 & 11,300 & 6.7 & 7.8 & 08470500 & 05/23/2006 21:15 & 29.6 & 6,860 & 9.0 & 8.0 \\
\hline 08470500 & 02/23/2006 11:55 & 19.7 & 12,300 & 6.7 & 7.8 & 08470500 & 05/23/2006 21:30 & 29.4 & 6,930 & 8.7 & 8.0 \\
\hline 08470500 & 02/23/2006 13:10 & 19.7 & 14,200 & 6.9 & 7.9 & 08470500 & 05/23/2006 22:45 & 29 & 6,990 & 8.2 & 7.9 \\
\hline 08470500 & 02/23/2006 13:25 & 19.7 & 14,400 & 7.0 & 7.9 & 08470500 & 05/23/2006 23:00 & 29 & 7,290 & 7.8 & 7.9 \\
\hline 08470500 & 02/23/2006 13:40 & 19.7 & 14,400 & 7.1 & 7.9 & 08470500 & 05/23/2006 23:15 & 28.8 & 7,050 & 7.7 & 7.9 \\
\hline 08470500 & $02 / 23 / 2006$ 13:55 & 19.7 & 14,400 & 7.1 & 7.9 & 08470500 & 05/23/2006 23:30 & 29 & 7,600 & 7.1 & 7.8 \\
\hline 08470500 & 02/23/2006 14:10 & 19.7 & 14,300 & 7.0 & 7.9 & 08470500 & 05/23/2006 23:45 & 28.8 & 7,370 & 7.4 & 7.9 \\
\hline 08470500 & 02/23/2006 14:25 & 19.7 & 14,500 & 7.0 & 7.9 & 08470500 & 05/24/2006 00:00 & 28.7 & 7,250 & 7.6 & 7.9 \\
\hline \multicolumn{6}{|c|}{ Station 08470500 May data-collection period } & 08470500 & 05/24/2006 00:15 & 28.7 & 7,360 & 7.3 & 7.9 \\
\hline 08470500 & 05/23/2006 10:45 & 27.5 & 8,970 & 4.3 & 7.6 & 08470500 & 05/24/2006 00:30 & 28.7 & 7,360 & 7.3 & 7.9 \\
\hline 08470500 & 05/23/2006 11:00 & 27.3 & 8,550 & 4.7 & 7.7 & 08470500 & 05/24/2006 00:45 & 28.7 & 7,540 & 6.6 & 7.8 \\
\hline 08470500 & 05/23/2006 11:15 & 27.2 & 8,370 & 4.6 & 7.6 & 08470500 & 05/24/2006 01:00 & 28.7 & 7,480 & 6.5 & 7.8 \\
\hline 08470500 & 05/23/2006 11:30 & 27.3 & 8,320 & 4.3 & 7.6 & 08470500 & 05/24/2006 01:15 & 28.5 & 7,450 & 6.6 & 7.8 \\
\hline 08470500 & 05/23/2006 13:45 & 28.6 & 7,930 & 5.8 & 7.7 & 08470500 & 05/24/2006 03:30 & 28.2 & 7,220 & 5.7 & 7.8 \\
\hline 08470500 & 05/23/2006 14:00 & 28.7 & 7,900 & 5.9 & 7.7 & 08470500 & 05/24/2006 03:45 & 28.2 & 7,280 & 5.6 & 7.8 \\
\hline 08470500 & 05/23/2006 14:15 & 28.9 & 7,810 & 5.9 & 7.7 & 08470500 & 05/24/2006 04:00 & 28.2 & 7,250 & 5.5 & 7.8 \\
\hline 08470500 & 05/23/2006 14:30 & 29.1 & 7,680 & 6.4 & 7.8 & 08470500 & 05/24/2006 04:15 & 28.2 & 7,250 & 5.2 & 7.7 \\
\hline 08470500 & 05/23/2006 14:45 & 29.2 & 7,670 & 6.9 & 7.8 & 08470500 & 05/24/2006 04:30 & 28.1 & 7,350 & 5.1 & 7.7 \\
\hline 08470500 & 05/23/2006 15:00 & 29.3 & 7,530 & 6.9 & 7.8 & 08470500 & 05/24/2006 04:45 & 28.1 & 7,330 & 5.1 & 7.7 \\
\hline 08470500 & 05/23/2006 15:15 & 29.4 & 7,420 & 7.1 & 7.8 & 08470500 & 05/24/2006 05:00 & 28.1 & 7,300 & 5.1 & 7.7 \\
\hline 08470500 & 05/23/2006 15:30 & 29.6 & 7,400 & 6.8 & 7.8 & 08470500 & 05/24/2006 05:15 & 28.1 & 7,470 & 4.8 & 7.7 \\
\hline 08470500 & 05/23/2006 15:45 & 29.7 & 7,280 & 6.8 & 7.8 & 08470500 & 05/24/2006 05:30 & 28.0 & 7,580 & 4.7 & 7.7 \\
\hline 08470500 & 05/23/2006 16:00 & 29.9 & 7,120 & 7.0 & 7.8 & 08470500 & 05/24/2006 05:45 & 28.0 & 7,590 & 4.6 & 7.7 \\
\hline 08470500 & 05/23/2006 16:15 & 29.8 & 7,100 & 7.2 & 7.8 & 08470500 & 05/24/2006 06:00 & 28.0 & 7,500 & 4.7 & 7.7 \\
\hline 08470500 & 05/23/2006 16:30 & 29.8 & 6,880 & 7.8 & 7.9 & 08470500 & 05/24/2006 06:15 & 27.9 & 7,440 & 4.5 & 7.7 \\
\hline 08470500 & 05/23/2006 16:45 & 30.0 & 6,870 & 7.9 & 7.9 & 08470500 & 05/24/2006 06:30 & 27.9 & 7,380 & 4.5 & 7.7 \\
\hline 08470500 & 05/23/2006 17:00 & 30.3 & 6,650 & 8.3 & 7.9 & 08470500 & 05/24/2006 06:45 & 27.9 & 7,370 & 4.3 & 7.7 \\
\hline 08470500 & 05/23/2006 17:15 & 30.2 & 6,780 & 8.2 & 7.9 & 08470500 & 05/24/2006 07:00 & 27.8 & 7,400 & 4.2 & 7.7 \\
\hline 08470500 & 05/23/2006 17:30 & 30.4 & 6,700 & 8.2 & 7.9 & 08470500 & 05/24/2006 07:15 & 27.8 & 7,390 & 4.2 & 7.7 \\
\hline 08470500 & 05/23/2006 17:45 & 30.4 & 6,720 & 8.2 & 7.9 & 08470500 & 05/24/2006 07:30 & 27.8 & 7,390 & 4.0 & 7.7 \\
\hline 08470500 & 05/23/2006 18:00 & 30.5 & 6,650 & 8.4 & 7.9 & 08470500 & 05/24/2006 07:45 & 27.8 & 7,390 & 4.0 & 7.7 \\
\hline 08470500 & 05/23/2006 18:15 & 30.6 & 6,510 & 8.7 & 8.0 & 08470500 & 05/24/2006 08:00 & 27.8 & 7,520 & 3.9 & 7.6 \\
\hline 08470500 & 05/23/2006 18:30 & 30.6 & 6,550 & 8.6 & 8.0 & 08470500 & 05/24/2006 08:15 & 27.7 & 7,400 & 4.0 & 7.7 \\
\hline 08470500 & 05/23/2006 18:45 & 30.4 & 6,710 & 8.6 & 7.9 & 08470500 & 05/24/2006 08:30 & 27.7 & 7,470 & 3.9 & 7.7 \\
\hline
\end{tabular}


Appendix 1-2.2. Data collected at near-surface depth of the Arroyo Colorado near Rio Hondo, Texas, using multiparameter continuous water-quality monitors-Continued.

\begin{tabular}{|c|c|c|c|c|c|c|c|c|c|c|c|}
\hline $\begin{array}{c}\text { USGS } \\
\text { station no. }\end{array}$ & $\begin{array}{c}\text { Date } \\
\text { and time }\end{array}$ & $\begin{array}{c}\text { Temp- } \\
\text { erature } \\
\left({ }^{\circ} \mathrm{C}\right)\end{array}$ & $\begin{array}{c}\text { Specific } \\
\text { conduc- } \\
\text { tance } \\
(\mu \mathrm{S} / \mathrm{cm})\end{array}$ & $\begin{array}{c}\begin{array}{c}\text { Dis- } \\
\text { solved } \\
\text { oxygen } \\
\text { (mg/L) }\end{array}\end{array}$ & $\begin{array}{c}\mathrm{pH} \\
\text { (stan- } \\
\text { dard } \\
\text { units) }\end{array}$ & $\begin{array}{c}\text { USGS } \\
\text { station no. }\end{array}$ & $\begin{array}{c}\text { Date } \\
\text { and time }\end{array}$ & $\begin{array}{l}\text { Temp- } \\
\text { erature } \\
\left({ }^{\circ} \mathrm{C}\right)\end{array}$ & $\begin{array}{c}\text { Specific } \\
\text { conduc- } \\
\text { tance } \\
(\mu \mathrm{S} / \mathrm{cm})\end{array}$ & $\begin{array}{c}\begin{array}{c}\text { Dis- } \\
\text { solved } \\
\text { oxygen } \\
\text { (mg/L) }\end{array}\end{array}$ & $\begin{array}{c}\mathrm{pH} \\
\text { (stan- } \\
\text { dard } \\
\text { units) }\end{array}$ \\
\hline 08470500 & 05/24/2006 08:45 & 27.7 & 12,000 & 2.4 & 7.5 & 08470520 & 02/22/2006 20:45 & 19.4 & 12,800 & 7.6 & 7.8 \\
\hline 08470500 & 05/24/2006 09:00 & 27.7 & 11,200 & 3.1 & 7.6 & 08470520 & 02/22/2006 21:00 & 19.5 & 12,700 & 7.7 & 7.8 \\
\hline 08470500 & 05/24/2006 09:15 & 27.7 & 10,900 & 2.9 & 7.6 & 08470520 & 02/22/2006 21:15 & 19.5 & 12,600 & 7.9 & 7.8 \\
\hline 08470500 & 05/24/2006 10:00 & 27.7 & 10,800 & 3.4 & 7.6 & 08470520 & 02/22/2006 22:00 & 19.4 & 13,100 & 7.7 & 7.8 \\
\hline 08470500 & 05/24/2006 10:15 & 27.8 & 10,500 & 3.8 & 7.7 & 08470520 & 02/22/2006 22:15 & 19.4 & 12,900 & 7.7 & 7.8 \\
\hline 08470500 & 05/24/2006 10:30 & 27.9 & 11,500 & 3.8 & 7.7 & 08470520 & 02/22/2006 22:30 & 19.4 & 12,700 & 7.8 & 7.8 \\
\hline 08470500 & 05/24/2006 10:45 & 28.0 & 10,700 & 4.6 & 7.7 & 08470520 & 02/22/2006 22:45 & 19.4 & 12,400 & 7.9 & 7.8 \\
\hline 08470500 & 05/24/2006 11:00 & 28.0 & 10,800 & 4.4 & 7.7 & 08470520 & 02/22/2006 23:00 & 19.4 & 12,300 & 8.0 & 7.8 \\
\hline 08470500 & 05/24/2006 11:15 & 28.1 & 10,700 & 5.6 & 7.8 & 08470520 & 02/22/2006 23:15 & 19.4 & 12,600 & 8.0 & 7.8 \\
\hline 08470500 & 05/24/2006 12:30 & 29.0 & 9,740 & 11.4 & 8.2 & 08470520 & 02/23/2006 00:30 & 19.4 & 12,600 & 8.3 & 7.8 \\
\hline 08470500 & 05/24/2006 12:45 & 29.1 & 9,950 & 12.9 & 8.3 & 08470520 & 02/23/2006 00:45 & 19.4 & 12,300 & 8.4 & 7.9 \\
\hline 08470500 & 05/24/2006 13:00 & 29.2 & 10,100 & 13 & 8.3 & 08470520 & 02/23/2006 01:00 & 19.4 & 12,600 & 8.4 & 7.8 \\
\hline 08470500 & 05/24/2006 13:15 & 29.3 & 10,000 & 13 & 8.3 & 08470520 & 02/23/2006 01:15 & 19.4 & 12,600 & 8.5 & 7.9 \\
\hline \multicolumn{6}{|c|}{ Station 08470520 February data-collection period } & 08470520 & 02/23/2006 01:30 & 19.4 & 12,500 & 8.7 & 7.9 \\
\hline 08470520 & 02/22/2006 12:00 & 19.3 & 13,000 & 7.4 & 7.8 & 08470520 & 02/23/2006 01:45 & 19.4 & 12,400 & 8.7 & 7.9 \\
\hline 08470520 & $02 / 22 / 200612: 15$ & 19.3 & 12,900 & 7.4 & 7.8 & 08470520 & 02/23/2006 02:00 & 19.4 & 12,500 & 8.8 & 7.9 \\
\hline 08470520 & $02 / 22 / 200612: 30$ & 19.3 & 13,200 & 7.3 & 7.8 & 08470520 & 02/23/2006 02:15 & 19.4 & 12,700 & 8.7 & 7.9 \\
\hline 08470520 & $02 / 22 / 2006$ 12:45 & 19.3 & 13,100 & 7.3 & 7.8 & 08470520 & 02/23/2006 02:30 & 19.3 & 13,000 & 7.4 & 7.8 \\
\hline 08470520 & 02/22/2006 13:00 & 19.3 & 13,000 & 7.4 & 7.8 & 08470520 & 02/23/2006 02:45 & 19.3 & 12,900 & 7.4 & 7.8 \\
\hline 08470520 & 02/22/2006 13:15 & 19.4 & 12,900 & 7.5 & 7.8 & 08470520 & 02/23/2006 03:00 & 19.3 & 13,200 & 7.3 & 7.8 \\
\hline 08470520 & $02 / 22 / 200615: 30$ & 19.4 & 12,400 & 7.9 & 7.8 & 08470520 & 02/23/2006 05:15 & 19.4 & 13,100 & 7.7 & 7.8 \\
\hline 08470520 & $02 / 22 / 200615: 45$ & 19.4 & 12,300 & 8.0 & 7.8 & 08470520 & 02/23/2006 05:30 & 19.4 & 12,900 & 7.7 & 7.8 \\
\hline 08470520 & 02/22/2006 16:00 & 19.4 & 12,600 & 8.0 & 7.8 & 08470520 & 02/23/2006 05:45 & 19.4 & 12,700 & 7.8 & 7.8 \\
\hline 08470520 & $02 / 22 / 200616: 15$ & 19.4 & 12,400 & 8.1 & 7.8 & 08470520 & 02/23/2006 06:00 & 19.4 & 12,400 & 7.9 & 7.8 \\
\hline 08470520 & $02 / 22 / 200616: 30$ & 19.4 & 12,400 & 8.2 & 7.8 & 08470520 & 02/23/2006 06:15 & 19.4 & 12,300 & 8.0 & 7.8 \\
\hline 08470520 & $02 / 22 / 200616: 45$ & 19.4 & 12,700 & 8.2 & 7.8 & 08470520 & 02/23/2006 06:30 & 19.4 & 12,600 & 8.0 & 7.8 \\
\hline 08470520 & 02/22/2006 17:00 & 19.4 & 12,600 & 8.3 & 7.8 & 08470520 & 02/23/2006 06:45 & 19.4 & 12,400 & 8.1 & 7.8 \\
\hline 08470520 & 02/22/2006 17:15 & 19.4 & 12,600 & 8.3 & 7.8 & 08470520 & 02/23/2006 07:00 & 19.4 & 12,400 & 8.2 & 7.8 \\
\hline 08470520 & $02 / 22 / 2006$ 17:30 & 19.4 & 12,300 & 8.4 & 7.9 & 08470520 & 02/23/2006 07:15 & 19.4 & 12,700 & 8.2 & 7.8 \\
\hline 08470520 & $02 / 22 / 2006$ 17:45 & 19.4 & 12,600 & 8.4 & 7.8 & 08470520 & 02/23/2006 07:30 & 19.4 & 12,600 & 8.3 & 7.8 \\
\hline 08470520 & 02/22/2006 18:00 & 19.4 & 12,600 & 8.5 & 7.9 & 08470520 & 02/23/2006 07:45 & 19.4 & 12,600 & 8.3 & 7.8 \\
\hline 08470520 & 02/22/2006 18:15 & 19.4 & 12,500 & 8.7 & 7.9 & 08470520 & 02/23/2006 08:00 & 19.4 & 12,300 & 8.4 & 7.9 \\
\hline 08470520 & $02 / 22 / 2006$ 18:30 & 19.4 & 12,400 & 8.7 & 7.9 & 08470520 & 02/23/2006 08:15 & 19.4 & 12,600 & 8.4 & 7.8 \\
\hline 08470520 & $02 / 22 / 2006$ 18:45 & 19.4 & 12,500 & 8.8 & 7.9 & 08470520 & 02/23/2006 08:30 & 19.4 & 12,600 & 8.5 & 7.9 \\
\hline 08470520 & 02/22/2006 19:00 & 19.4 & 12,700 & 8.7 & 7.9 & 08470520 & 02/23/2006 08:45 & 19.4 & 12,500 & 8.7 & 7.9 \\
\hline 08470520 & 02/22/2006 19:15 & 19.3 & 13,000 & 7.4 & 7.8 & 08470520 & 02/23/2006 09:00 & 19.4 & 12,400 & 8.7 & 7.9 \\
\hline 08470520 & $02 / 22 / 200619: 30$ & 19.3 & 12,900 & 7.4 & 7.8 & 08470520 & 02/23/2006 09:15 & 19.4 & 12,500 & 8.8 & 7.9 \\
\hline 08470520 & 02/22/2006 19:45 & 19.3 & 13,200 & 7.3 & 7.8 & 08470520 & 02/23/2006 09:30 & 19.4 & 12,700 & 8.7 & 7.9 \\
\hline 08470520 & 02/22/2006 20:00 & 19.3 & 13,100 & 7.3 & 7.8 & 08470520 & 02/23/2006 09:45 & 19.3 & 13,000 & 7.4 & 7.8 \\
\hline 08470520 & 02/22/2006 20:15 & 19.3 & 13,000 & 7.4 & 7.8 & 08470520 & 02/23/2006 10:00 & 19.3 & 12,900 & 7.4 & 7.8 \\
\hline 08470520 & $02 / 22 / 200620: 30$ & 19.4 & 12,900 & 7.5 & 7.8 & 08470520 & 02/23/2006 10:15 & 19.3 & 13,200 & 7.3 & 7.8 \\
\hline
\end{tabular}


Appendix 1-2.2. Data collected at near-surface depth of the Arroyo Colorado near Rio Hondo, Texas, using multiparameter continuous water-quality monitors-Continued.

\begin{tabular}{|c|c|c|c|c|c|c|c|c|c|c|c|}
\hline $\begin{array}{c}\text { USGS } \\
\text { station no. }\end{array}$ & $\begin{array}{c}\text { Date } \\
\text { and time }\end{array}$ & $\begin{array}{l}\text { Temp- } \\
\text { erature } \\
\left({ }^{\circ} \mathrm{C}\right)\end{array}$ & $\begin{array}{l}\text { Specific } \\
\text { conduc- } \\
\text { tance } \\
(\mu \mathrm{S} / \mathrm{cm})\end{array}$ & $\begin{array}{l}\text { Dis- } \\
\text { solved } \\
\text { oxygen } \\
\text { (mg/L) }\end{array}$ & $\begin{array}{l}\mathrm{pH} \\
\text { (stan- } \\
\text { dard } \\
\text { units) }\end{array}$ & $\begin{array}{c}\text { USGS } \\
\text { station no. }\end{array}$ & $\begin{array}{c}\text { Date } \\
\text { and time }\end{array}$ & $\begin{array}{l}\text { Temp- } \\
\text { erature } \\
\left({ }^{\circ} \mathrm{C}\right)\end{array}$ & $\begin{array}{c}\text { Specific } \\
\text { conduc- } \\
\text { tance } \\
(\mu \mathrm{S} / \mathrm{cm})\end{array}$ & $\begin{array}{l}\text { Dis- } \\
\text { solved } \\
\text { oxygen } \\
\text { (mg/L) }\end{array}$ & $\begin{array}{l}\mathrm{pH} \\
\text { (stan- } \\
\text { dard } \\
\text { units) }\end{array}$ \\
\hline 08470520 & 02/23/2006 10:30 & 19.3 & 13,100 & 7.3 & 7.8 & 08470520 & 05/23/2006 19:15 & 31.2 & 7,990 & 19.1 & 8.6 \\
\hline 08470520 & 02/23/2006 10:45 & 19.3 & 13,000 & 7.4 & 7.8 & 08470520 & 05/23/2006 19:30 & 31.3 & 8,360 & 21.7 & 8.7 \\
\hline 08470520 & 02/23/2006 11:00 & 19.4 & 12,900 & 7.5 & 7.8 & 08470520 & 05/23/2006 19:45 & 31.1 & 8,420 & 20.7 & 8.7 \\
\hline 08470520 & 02/23/2006 11:45 & 19.5 & 12,600 & 7.9 & 7.8 & 08470520 & 05/23/2006 20:30 & 31.0 & 8,350 & 19.9 & 8.6 \\
\hline 08470520 & 02/23/2006 12:00 & 19.4 & 12,700 & 7.8 & 7.8 & 08470520 & 05/23/2006 20:45 & 30.4 & 8,460 & 17.8 & 8.5 \\
\hline 08470520 & 02/23/2006 12:15 & 19.4 & 12,700 & 7.8 & 7.8 & 08470520 & 05/23/2006 21:00 & 30.5 & 8,180 & 18.5 & 8.5 \\
\hline 08470520 & $02 / 23 / 200612: 30$ & 19.4 & 13,100 & 7.7 & 7.8 & 08470520 & 05/23/2006 21:15 & 30.4 & 8,260 & 17.7 & 8.5 \\
\hline 08470520 & 02/23/2006 12:45 & 19.4 & 12,900 & 7.7 & 7.8 & 08470520 & 05/23/2006 21:30 & 30.0 & 8,120 & 14.8 & 8.4 \\
\hline 08470520 & 02/23/2006 13:00 & 19.4 & 12,700 & 7.8 & 7.8 & 08470520 & 05/23/2006 21:45 & 30.1 & 8,200 & 14.9 & 8.4 \\
\hline 08470520 & 02/23/2006 14:15 & 19.4 & 12,400 & 8.2 & 7.8 & 08470520 & 05/23/2006 23:00 & 29.5 & 8,500 & 12.5 & 8.2 \\
\hline 08470520 & 02/23/2006 14:30 & 19.4 & 12,700 & 8.2 & 7.8 & 08470520 & 05/23/2006 23:15 & 29.4 & 8,500 & 12.1 & 8.2 \\
\hline 08470520 & $02 / 23 / 200614: 45$ & 19.4 & 12,600 & 8.3 & 7.8 & 08470520 & 05/23/2006 23:30 & 29.4 & 8,540 & 11.8 & 8.2 \\
\hline \multicolumn{6}{|c|}{ Station 08470520 May data-collection period } & 08470520 & 05/23/2006 23:45 & 29.4 & 8,520 & 12.0 & 8.2 \\
\hline 08470520 & 05/23/2006 10:15 & 27.2 & 7,220 & 7.9 & 7.9 & 08470520 & 05/24/2006 00:00 & 29.5 & 8,560 & 12.3 & 8.2 \\
\hline 08470520 & 05/23/2006 10:30 & 27.3 & 7,560 & 9.3 & 8.0 & 08470520 & 05/24/2006 00:15 & 29.3 & 8,480 & 11.8 & 8.2 \\
\hline 08470520 & 05/23/2006 10:45 & 27.5 & 7,980 & 8.8 & 8.0 & 08470520 & 05/24/2006 00:30 & 29.3 & 8,520 & 11.6 & 8.2 \\
\hline 08470520 & 05/23/2006 11:00 & 27.8 & 8,320 & 8.3 & 7.9 & 08470520 & 05/24/2006 00:45 & 29.3 & 8,500 & 11.3 & 8.2 \\
\hline 08470520 & 05/23/2006 11:15 & 28.0 & 8,590 & 8.6 & 8.0 & 08470520 & 05/24/2006 01:00 & 29.2 & 8,470 & 11.3 & 8.2 \\
\hline 08470520 & 05/23/2006 11:30 & 28.1 & 8,580 & 10.4 & 8.1 & 08470520 & 05/24/2006 01:15 & 29.2 & 8,410 & 11.0 & 8.2 \\
\hline 08470520 & 05/23/2006 11:45 & 28.1 & 8,610 & 10.7 & 8.1 & 08470520 & 05/24/2006 01:30 & 29.2 & 8,460 & 11.0 & 8.2 \\
\hline 08470520 & 05/23/2006 14:00 & 28.8 & 8,480 & 17.1 & 8.5 & 08470520 & 05/24/2006 03:45 & 28.8 & 8,350 & 10.3 & 8.1 \\
\hline 08470520 & 05/23/2006 14:15 & 29.1 & 8,460 & 18.3 & 8.6 & 08470520 & 05/24/2006 04:00 & 28.8 & 8,360 & 10.2 & 8.1 \\
\hline 08470520 & 05/23/2006 14:30 & 29.4 & 8,400 & 18.9 & 8.6 & 08470520 & 05/24/2006 04:15 & 28.9 & 8,420 & 10.2 & 8.1 \\
\hline 08470520 & 05/23/2006 14:45 & 29.5 & 8,400 & 19.6 & 8.7 & 08470520 & 05/24/2006 04:30 & 28.8 & 8,430 & 10.2 & 8.1 \\
\hline 08470520 & 05/23/2006 15:00 & 29.5 & 8,460 & 19.5 & 8.7 & 08470520 & 05/24/2006 04:45 & 28.8 & 8,370 & 10.2 & 8.1 \\
\hline 08470520 & 05/23/2006 15:15 & 30.1 & 8,360 & 19.3 & 8.7 & 08470520 & 05/24/2006 05:00 & 28.7 & 8,380 & 10.2 & 8.1 \\
\hline 08470520 & 05/23/2006 15:30 & 29.8 & 8,470 & 19.1 & 8.6 & 08470520 & 05/24/2006 05:15 & 28.7 & 8,410 & 10.1 & 8.1 \\
\hline 08470520 & 05/23/2006 15:45 & 29.4 & 8,510 & 13.7 & 8.3 & 08470520 & 05/24/2006 05:30 & 28.6 & 8,400 & 9.9 & 8.1 \\
\hline 08470520 & 05/23/2006 16:00 & 29.5 & 8,530 & 15.7 & 8.4 & 08470520 & 05/24/2006 05:45 & 28.6 & 8,340 & 9.8 & 8.1 \\
\hline 08470520 & 05/23/2006 16:15 & 29.9 & 8,580 & 15.5 & 8.5 & 08470520 & 05/24/2006 06:00 & 28.5 & 8,320 & 9.6 & 8.1 \\
\hline 08470520 & 05/23/2006 16:30 & 30.0 & 8,560 & 16.3 & 8.5 & 08470520 & 05/24/2006 06:15 & 28.4 & 8,200 & 9.5 & 8.1 \\
\hline 08470520 & 05/23/2006 16:45 & 30.2 & 8,590 & 17.1 & 8.5 & 08470520 & 05/24/2006 06:30 & 28.4 & 8,240 & 9.3 & 8.0 \\
\hline 08470520 & 05/23/2006 17:00 & 30.4 & 8,600 & 19.5 & 8.6 & 08470520 & 05/24/2006 06:45 & 28.4 & 8,230 & 9.0 & 8.0 \\
\hline 08470520 & 05/23/2006 17:15 & 30.5 & 8,720 & 18.5 & 8.6 & 08470520 & 05/24/2006 07:00 & 28.3 & 8,150 & 8.7 & 8.0 \\
\hline 08470520 & 05/23/2006 17:30 & 30.8 & 8,640 & 21.6 & 8.7 & 08470520 & 05/24/2006 07:15 & 28.2 & 8,080 & 8.4 & 8.0 \\
\hline 08470520 & 05/23/2006 17:45 & 30.6 & 8,660 & 19.8 & 8.6 & 08470520 & 05/24/2006 07:30 & 28.0 & 7,960 & 8.0 & 8.0 \\
\hline 08470520 & 05/23/2006 18:00 & 31.0 & 8,220 & 18.8 & 8.6 & 08470520 & 05/24/2006 07:45 & 28.0 & 8,020 & 8.0 & 8.0 \\
\hline 08470520 & 05/23/2006 18:15 & 31.1 & 8,190 & 20.5 & 8.7 & 08470520 & 05/24/2006 08:00 & 28.0 & 8,110 & 8.1 & 7.9 \\
\hline 08470520 & 05/23/2006 18:30 & 31.5 & 7,970 & 20.2 & 8.6 & 08470520 & 05/24/2006 08:15 & 28.3 & 8,320 & 7.9 & 7.9 \\
\hline 08470520 & 05/23/2006 18:45 & 31.0 & 8,440 & 21.3 & 8.7 & 08470520 & 05/24/2006 08:30 & 28.1 & 8,170 & 7.8 & 7.9 \\
\hline 08470520 & 05/23/2006 19:00 & 31.6 & 8,180 & 21.7 & 8.7 & 08470520 & 05/24/2006 08:45 & 28.1 & 8,250 & 7.7 & 7.9 \\
\hline
\end{tabular}




\section{Water-Quality and Ancillary Data Collected From the Arroyo Colorado Near Rio Hondo, Texas, 2006}

Appendix 1-2.2. Data collected at near-surface depth of the Arroyo Colorado near Rio Hondo, Texas, using multiparameter continuous water-quality monitors-Continued.

\begin{tabular}{|c|c|c|c|c|c|c|c|c|c|c|c|}
\hline $\begin{array}{c}\text { USGS } \\
\text { station no. }\end{array}$ & $\begin{array}{c}\text { Date } \\
\text { and time }\end{array}$ & $\begin{array}{c}\text { Temp- } \\
\text { erature } \\
\left({ }^{\circ} \mathrm{C}\right)\end{array}$ & $\begin{array}{c}\text { Specific } \\
\text { conduc- } \\
\text { tance } \\
(\mu \mathrm{S} / \mathrm{cm})\end{array}$ & $\begin{array}{l}\text { Dis- } \\
\text { solved } \\
\text { oxygen } \\
\text { (mg/L) }\end{array}$ & $\begin{array}{c}\mathrm{pH} \\
\text { (stan- } \\
\text { dard } \\
\text { units) }\end{array}$ & $\begin{array}{c}\text { USGS } \\
\text { station no. }\end{array}$ & $\begin{array}{c}\text { Date } \\
\text { and time }\end{array}$ & $\begin{array}{c}\text { Temp- } \\
\text { erature } \\
\left({ }^{\circ} \mathrm{C}\right)\end{array}$ & $\begin{array}{c}\text { Specific } \\
\text { conduc- } \\
\text { tance } \\
(\mu S / \mathrm{cm})\end{array}$ & $\begin{array}{l}\text { Dis- } \\
\text { solved } \\
\text { oxygen } \\
\text { (mg/L) }\end{array}$ & $\begin{array}{c}\mathrm{pH} \\
\text { (stan- } \\
\text { dard } \\
\text { units) }\end{array}$ \\
\hline \multicolumn{6}{|c|}{ Station 08470520 May data-collection period-Continued } & \multicolumn{6}{|c|}{ Station 08470520 May data-collection period-Continued } \\
\hline 08470520 & 05/24/2006 09:00 & 28.1 & 8,220 & 8.0 & 7.9 & 08470520 & $05 / 24 / 200611: 30$ & 28.4 & 11,400 & 7.3 & 7.9 \\
\hline 08470520 & 05/24/2006 09:15 & 28.3 & 8,770 & 7.9 & 7.9 & 08470520 & $05 / 24 / 200611: 45$ & 28.4 & 11,400 & 7.6 & 7.9 \\
\hline 08470520 & 05/24/2006 09:30 & 28.2 & 10,800 & 6.6 & 7.8 & 08470520 & 05/24/2006 12:00 & 28.7 & 10,900 & 9.5 & 8.0 \\
\hline 08470520 & 05/24/2006 09:45 & 27.8 & 11,300 & 6.4 & 7.8 & 08470520 & $05 / 24 / 200612: 15$ & 28.7 & 10,900 & 10.9 & 8.1 \\
\hline 08470520 & 05/24/2006 10:00 & 27.8 & 10,900 & 6.6 & 7.8 & 08470520 & $05 / 24 / 200612: 30$ & 28.8 & 10,900 & 10.4 & 8.1 \\
\hline 08470520 & 05/24/2006 10:15 & 27.8 & 10,400 & 6.4 & 7.8 & 08470520 & $05 / 24 / 200612: 45$ & 28.8 & 10,800 & 13.1 & 8.2 \\
\hline 08470520 & 05/24/2006 10:30 & 28.0 & 10,600 & 6.7 & 7.8 & 08470520 & 05/24/2006 13:00 & 29.0 & 10,700 & 14.2 & 8.3 \\
\hline 08470520 & 05/24/2006 10:45 & 28.1 & 10,800 & 7.0 & 7.8 & 08470520 & 05/24/2006 13:15 & 29.1 & 11,200 & 11.7 & 8.2 \\
\hline 08470520 & 05/24/2006 11:00 & 28.2 & 11,500 & 7.2 & 7.9 & 08470520 & $05 / 24 / 200613: 30$ & 29.1 & 11,000 & 14.6 & 8.3 \\
\hline 08470520 & $05 / 24 / 200611: 15$ & 28.3 & 11,700 & 7.7 & 7.9 & & & & & & \\
\hline
\end{tabular}

Appendix 1-2.3.a. Data collected at near-surface depth of the Arroyo Colorado near Rio Hondo, Texas, using algal growth and mortality analyses/bioassay analysis.

$\left[+\mathrm{N}\right.$, nitrogen addition; $+\mathrm{NP}$, nitrogen and phosphorus addition; $+\mathrm{P}$, phosphorus addition; control, no addition. $\mathrm{R}^{2}$, statistical measure of the degree to which two different things are related. An $\mathrm{R}^{2}$ of 1.00 indicates perfect correlation; an $\mathrm{R}^{2}$ of 0 indicates no correlation. A negative growth rate indicates that phytoplankton are being consumed by zooplankton and is an algal mortality rate]

\begin{tabular}{cclcc}
\hline $\begin{array}{c}\text { USGS } \\
\text { station no. }\end{array}$ & $\begin{array}{c}\text { Date and } \\
\text { time }\end{array}$ & Sample type & Average $^{2}$ & $\begin{array}{c}\text { Average } \\
\text { growth rate }\end{array}$ \\
\hline 08470450 & $02 / 22 / 200617: 30$ & $+\mathrm{N}$ & 0.95 & 0.556 \\
08470450 & $02 / 23 / 200617: 30$ & $+\mathrm{NP}$ & .96 & .430 \\
08470450 & $02 / 24 / 200617: 30$ & $+\mathrm{P}$ & .98 & .422 \\
08470450 & $02 / 25 / 200617: 30$ & control & .96 & .556 \\
08470450 & $05 / 23 / 200611: 30$ & $+\mathrm{N}$ & .74 & -.285 \\
08470450 & $05 / 24 / 200611: 30$ & $+\mathrm{NP}$ & .18 & .166 \\
08470450 & $05 / 25 / 200611: 30$ & $+\mathrm{P}$ & .89 & -.470 \\
08470450 & $05 / 26 / 200611: 30$ & control & .78 & -.398 \\
08470460 & $02 / 22 / 200616: 30$ & $+\mathrm{N}$ & .90 & .315 \\
08470460 & $02 / 23 / 200616: 30$ & $+\mathrm{NP}$ & .91 & .415 \\
08470460 & $02 / 24 / 200616: 30$ & $+\mathrm{P}$ & .90 & .315 \\
08470460 & $02 / 25 / 200616: 30$ & control & .92 & .553 \\
08470460 & $05 / 23 / 200612: 30$ & $+\mathrm{N}$ & .77 & .519 \\
08470460 & $05 / 24 / 200612: 30$ & $+\mathrm{NP}$ & .32 & .195 \\
08470460 & $05 / 25 / 200612: 30$ & $+\mathrm{P}$ & .39 & .220 \\
08470460 & $05 / 26 / 200612: 30$ & control & .76 & .493
\end{tabular}

\begin{tabular}{cclcc}
\hline $\begin{array}{c}\text { USGS } \\
\text { station no. }\end{array}$ & $\begin{array}{c}\text { Date and } \\
\text { time }\end{array}$ & Sample type & Average $\mathrm{R}^{2}$ & $\begin{array}{c}\text { Average } \\
\text { growth rate }\end{array}$ \\
\hline 08470520 & $02 / 25 / 200613: 30$ & control & 0.86 & 0.511 \\
08470500 & $02 / 22 / 200615: 30$ & $+\mathrm{N}$ & .86 & .512 \\
08470500 & $02 / 23 / 200615: 30$ & $+\mathrm{NP}$ & .82 & .398 \\
08470500 & $02 / 24 / 200615: 30$ & $+\mathrm{P}$ & .82 & .353 \\
08470500 & $02 / 25 / 200615: 30$ & control & .65 & .325 \\
08470500 & $05 / 23 / 200613: 30$ & $+\mathrm{N}$ & .92 & -.255 \\
08470500 & $05 / 24 / 200613: 30$ & $+\mathrm{NP}$ & .54 & -.193 \\
08470500 & $05 / 25 / 200613: 30$ & $+\mathrm{P}$ & .78 & -.235 \\
08470500 & $05 / 26 / 200613: 30$ & control & .86 & -.209 \\
08470520 & $02 / 22 / 200613: 30$ & $+\mathrm{N}$ & .86 & .498 \\
08470520 & $02 / 23 / 200613: 30$ & $+\mathrm{NP}$ & .87 & .440 \\
08470520 & $02 / 24 / 200613: 30$ & $+\mathrm{P}$ & .85 & .455 \\
08470520 & $05 / 23 / 200614: 30$ & $+\mathrm{N}$ & .94 & -.290 \\
08470520 & $05 / 24 / 200614: 30$ & $+\mathrm{NP}$ & .57 & -.189 \\
08470520 & $05 / 25 / 200614: 30$ & $+\mathrm{P}$ & .95 & -.332 \\
08470520 & $05 / 26 / 200614: 30$ & control & .93 & -.377 \\
\hline
\end{tabular}


Appendix 1-2.3.b. Data collected at near-surface depth of the Arroyo Colorado near Rio Hondo, Texas, using algal growth and mortality analyses/phytoplankton analysis.

[Division, type of phytoplankton; Taxa, a classification or group of organisms (for example, kingdom, phylum, class, order, family, genus, species—becomes more specific with increasing number; cells/mL, cells per milliliter of sample; sp, unknown species; --, not applicable; <, less than; mm, millimeter; >, more than]

\begin{tabular}{|c|c|c|c|c|c|c|}
\hline $\begin{array}{c}\text { USGS } \\
\text { station no. }\end{array}$ & $\begin{array}{l}\text { Date and } \\
\text { time }\end{array}$ & Division & Taxa1 & Taxa2 & $\begin{array}{c}\text { Count } \\
\text { (cellls } / \mathrm{mL} \text { ) }\end{array}$ & $\begin{array}{c}\text { Sample } \\
\text { total } \\
\text { (cells } / \mathrm{mL} \text { ) }\end{array}$ \\
\hline \multicolumn{6}{|c|}{ Station 08470450 February data-collection period } & 3,969 \\
\hline 08470450 & $02 / 22 / 200617: 30$ & Bacillariophyceae & Surirella & $\mathrm{sp}$ & 142 & \\
\hline 08470450 & 02/22/2006 17:30 & Bacillariophyceae & Pennate & -- & 142 & \\
\hline 08470450 & $02 / 22 / 200617: 30$ & Bacteria & spirillum & -- & 142 & \\
\hline 08470450 & $02 / 22 / 200617: 30$ & Chlorophyta & Closterium & $\mathrm{sp}$ & 142 & \\
\hline 08470450 & $02 / 22 / 200617: 30$ & Chlorophyta & Crucigenia & $\mathrm{sp}$ & 2,267 & \\
\hline 08470450 & $02 / 22 / 200617: 30$ & Chlorophyta & Monoraphidium & $\mathrm{sp}$ & 283 & \\
\hline 08470450 & $02 / 22 / 200617: 30$ & Cyanobacteria & coccoid & $<5 \mathrm{~mm}$ & 709 & \\
\hline 08470450 & 02/22/2006 17:30 & Indeterminate & coccoid & $>5 \mathrm{~mm}$ & 142 & \\
\hline \multicolumn{6}{|c|}{ Station 08470450 May data-collection period } & 23,241 \\
\hline 08470450 & 05/23/2006 11:30 & Bacillariophyceae & centric & small & 992 & \\
\hline 08470450 & 05/23/2006 11:30 & Bacillariophyceae & centric & large & 142 & \\
\hline 08470450 & 05/23/2006 11:30 & Bacillariophyceae & pennate & small & 1,417 & \\
\hline 08470450 & 05/23/2006 11:30 & Bacillariophyceae & Placoneis & $\mathrm{sp}$ & 142 & \\
\hline 08470450 & 05/23/2006 11:30 & Chlorophyta & coccoid & $<5 \mathrm{~mm}$ & 567 & \\
\hline 08470450 & 05/23/2006 11:30 & Chlorophyta & coccoid & $>5 \mathrm{~mm}$ & 142 & \\
\hline 08470450 & 05/23/2006 11:30 & Chlorophyta & ovoid & -- & 142 & \\
\hline 08470450 & 05/23/2006 11:30 & Chlorophyta & Euglena & $\mathrm{sp}$ & 283 & \\
\hline 08470450 & 05/23/2006 11:30 & Chlorophyta & Kirchneriella & $\mathrm{sp}$ & 142 & \\
\hline 08470450 & 05/23/2006 11:30 & Chlorophyta & Monoraphidium & $\mathrm{sp}$ & 425 & \\
\hline 08470450 & 05/23/2006 11:30 & Chlorophyta & Oocystis & $\mathrm{sp}$ & 567 & \\
\hline 08470450 & 05/23/2006 11:30 & Chlorophyta & Scenedesmus & quadricauda & 1,700 & \\
\hline 08470450 & 05/23/2006 11:30 & Chlorophyta & Scenedesmus & $\mathrm{sp}$ & 8,502 & \\
\hline 08470450 & 05/23/2006 11:30 & Chlorophyta & Tetraedron & $\mathrm{sp}$ & 142 & \\
\hline 08470450 & 05/23/2006 11:30 & Chlorophyta & Trachelomonas & $\mathrm{sp}$ & 142 & \\
\hline 08470450 & 05/23/2006 11:30 & Cyanobacteria & coccoid & $<5 \mathrm{~mm}$ & 709 & \\
\hline 08470450 & 05/23/2006 11:30 & Cyanobacteria & colony & -- & 2,267 & \\
\hline 08470450 & 05/23/2006 11:30 & Cyanobacteria & spirillum & -- & 425 & \\
\hline 08470450 & 05/23/2006 11:30 & Cyanobacteria & lunate & -- & 1,417 & \\
\hline 08470450 & 05/23/2006 11:30 & Cyanobacteria & Gloeocapsa & punctata & 283 & \\
\hline 08470450 & 05/23/2006 11:30 & Indeterminate & coccoid & small & 142 & \\
\hline 08470450 & 05/23/2006 11:30 & Indeterminate & coccoid & large & 2,409 & \\
\hline 08470450 & 05/23/2006 11:30 & Indeterminate & ovate & -- & 142 & \\
\hline \multicolumn{6}{|c|}{ Station 08470460 February data-collection period } & 1,134 \\
\hline 08470460 & 02/22/2006 16:30 & Cyanobacteria & coccoid & $<5 \mathrm{~mm}$ & 1,134 & \\
\hline \multicolumn{6}{|c|}{ Station 08470460 May data-collection period } & 13,604 \\
\hline 08470460 & 05/23/2006 12:30 & Bacillariophyceae & pennate & -- & 142 & \\
\hline 08470460 & $05 / 23 / 200612: 30$ & Bacteria & spirillum & -- & 709 & \\
\hline 08470460 & 05/23/2006 12:30 & Chlorophyta & Ankistrodesmus & $\mathrm{sp}$ & 142 & \\
\hline 08470460 & 05/23/2006 12:30 & Chlorophyta & Kirchneriella & $\mathrm{sp}$ & 283 & \\
\hline 08470460 & 05/23/2006 12:30 & Chlorophyta & Monoraphidium & $\mathrm{sp}$ & 142 & \\
\hline 08470460 & $05 / 23 / 200612: 30$ & Chlorophyta & Scenedesmus & acuminatus & 1,134 & \\
\hline 08470460 & 05/23/2006 12:30 & Chlorophyta & Scenedesmus & $\mathrm{sp}$ & 3,401 & \\
\hline 08470460 & $05 / 23 / 200612: 30$ & Chlorophyta & coccoid & $<5 \mathrm{~mm}$ & 283 & \\
\hline 08470460 & 05/23/2006 12:30 & Cryptophyceae & cryptomonad & -- & 142 & \\
\hline 08470460 & 05/23/2006 12:30 & Cyanobacteria & coccoid & $<5 \mathrm{~mm}$ & 3,826 & \\
\hline 08470460 & $05 / 23 / 200612: 30$ & Cyanobacteria & coccoid & $>5 \mathrm{~mm}$ & 142 & \\
\hline 08470460 & 05/23/2006 12:30 & Cyanobacteria & spirillum & -- & 1,134 & \\
\hline 08470460 & 05/23/2006 12:30 & Cyanobacteria & short rod & -- & 283 & \\
\hline 08470460 & 05/23/2006 12:30 & Cyanobacteria & lunate & -- & 1,275 & \\
\hline 08470460 & 05/23/2006 12:30 & Cyanobacteria & filament & filament & 283 & \\
\hline 08470460 & 05/23/2006 12:30 & Indeterminate & coccoid & $>5 \mathrm{~mm}$ & 283 & \\
\hline
\end{tabular}




\section{Water-Quality and Ancillary Data Collected From the Arroyo Colorado Near Rio Hondo, Texas, 2006}

Appendix 1-2.3.b. Data collected at near-surface depth of the Arroyo Colorado near Rio Hondo, Texas, using algal growth and mortality analyses/phytoplankton analysis-Continued.

\begin{tabular}{|c|c|c|c|c|c|c|}
\hline $\begin{array}{c}\text { USGS } \\
\text { station no. }\end{array}$ & $\begin{array}{l}\text { Date and } \\
\text { time }\end{array}$ & Division & Taxa1 & Taxa2 & $\begin{array}{c}\text { Count } \\
\text { (cells/mL) }\end{array}$ & $\begin{array}{c}\text { Sample } \\
\text { total } \\
\text { (cells } / \mathrm{mL} \text { ) }\end{array}$ \\
\hline \multicolumn{6}{|c|}{ Station 08470500 February data-collection period } & 2,056 \\
\hline 08470500 & 02/22/2006 15:30 & Chlorophyta & Kirchneriella & $\mathrm{sp}$ & 142 & \\
\hline 08470500 & 02/22/2006 15:30 & Cyanobacteria & coccoid & $<5 \mathrm{~mm}$ & 567 & \\
\hline 08470500 & $02 / 22 / 200615: 30$ & Cyanobacteria & filament & $1 \mathrm{~mm} \mathrm{dia}$ & 709 & \\
\hline 08470500 & $02 / 22 / 200615: 30$ & Cyanobacteria & lunate & -- & 71 & \\
\hline 08470500 & $02 / 22 / 200615: 30$ & Cyanobacteria & Oscillatoria & $\mathrm{sp}$ & 567 & \\
\hline \multicolumn{6}{|c|}{ Station 08470500 May data-collection period } & 171,180 \\
\hline 08470500 & 05/23/2006 13:30 & Bacillariophyceae & centric & small & 3,684 & \\
\hline 08470500 & 05/23/2006 13:30 & Bacillariophyceae & centric & large & 283 & \\
\hline 08470500 & 05/23/2006 13:30 & Bacillariophyceae & Nitzschia & $\mathrm{sp}$ & 283 & \\
\hline 08470500 & 05/23/2006 13:30 & Bacteria & filament & -- & 4,251 & \\
\hline 08470500 & 05/23/2006 13:30 & Bacteria & spirillum & -- & 1,700 & \\
\hline 08470500 & 05/23/2006 13:30 & Chlorophyta & ovate & small & 2,834 & \\
\hline 08470500 & 05/23/2006 13:30 & Chlorophyta & ovate & large & 1,134 & \\
\hline 08470500 & 05/23/2006 13:30 & Chlorophyta & ovoid & -- & 283 & \\
\hline 08470500 & 05/23/2006 13:30 & Chlorophyta & paired cells & -- & 2,267 & \\
\hline 08470500 & 05/23/2006 13:30 & Chlorophyta & Ankistrodesmus & $\mathrm{sp}$ & 1,134 & \\
\hline 08470500 & 05/23/2006 13:30 & Chlorophyta & Kirchneriella & $\mathrm{sp}$ & 850 & \\
\hline 08470500 & 05/23/2006 13:30 & Chlorophyta & Scenedesmus & $\mathrm{sp}$ & 1,134 & \\
\hline 08470500 & 05/23/2006 13:30 & Cryptophyceae & cryptomonad & -- & 567 & \\
\hline 08470500 & 05/23/2006 13:30 & Cyanobacteria & coccoid & $<5 \mathrm{~mm}$ & 4,251 & \\
\hline 08470500 & 05/23/2006 13:30 & Cyanobacteria & short rod & -- & 1,417 & \\
\hline 08470500 & 05/23/2006 13:30 & Cyanobacteria & spirillum & -- & 1,134 & \\
\hline 08470500 & 05/23/2006 13:30 & Cyanobacteria & lunate & -- & 283 & \\
\hline 08470500 & 05/23/2006 13:30 & Cyanobacteria & filament & -- & 1,417 & \\
\hline 08470500 & 05/23/2006 13:30 & Cyanobacteria & Merismopedia & $\mathrm{sp}$ & 99,762 & \\
\hline 08470500 & 05/23/2006 13:30 & Indeterminate & coccoid & small & 40,812 & \\
\hline 08470500 & 05/23/2006 13:30 & Indeterminate & ovate & large & 283 & \\
\hline 08470500 & 05/23/2006 13:30 & Pyrrophyta & dinoflagellate & -- & 1,417 & \\
\hline \multicolumn{6}{|c|}{ Station 08470520 February data-collection period } & 1,843 \\
\hline 08470520 & 02/22/2006 13:30 & Bacillariophyceae & pennate & -- & 71 & \\
\hline 08470520 & 02/22/2006 13:30 & Chlorophyta & Kirchneriella & $\mathrm{sp}$ & 425 & \\
\hline 08470520 & 02/22/2006 13:30 & Chlorophyta & Monoraphidium & $\mathrm{sp}$ & 142 & \\
\hline 08470520 & 02/22/2006 13:30 & Cyanobacteria & coccoid & $<5 \mathrm{~mm}$ & 638 & \\
\hline 08470520 & 02/22/2006 13:30 & Cyanobacteria & short rod & -- & 142 & \\
\hline 08470520 & $02 / 22 / 200613: 30$ & Indeterminate & coccoid & $>5 \mathrm{~mm}$ & 354 & \\
\hline 08470520 & 02/22/2006 13:30 & Indeterminate & ovate & -- & 71 & \\
\hline \multicolumn{6}{|c|}{ Station 08470520 May data-collection period } & 264,143 \\
\hline 08470520 & 05/23/2006 14:30 & Bacillariophyceae & pennate & small & 567 & \\
\hline 08470520 & 05/23/2006 14:30 & Bacillariophyceae & centric & small & 3,401 & \\
\hline 08470520 & 05/23/2006 14:30 & Bacillariophyceae & centric & large & 567 & \\
\hline 08470520 & 05/23/2006 14:30 & Cyanobacteria & coccoid & $<5 \mathrm{~mm}$ & 1,700 & \\
\hline 08470520 & 05/23/2006 14:30 & Cyanobacteria & lunate & -- & 567 & \\
\hline 08470520 & 05/23/2006 14:30 & Indeterminate & ovate & $<5 \mathrm{~mm}$ & 1,134 & \\
\hline 08470520 & 05/23/2006 14:30 & Indeterminate & ovate & $>5 \mathrm{~mm}$ & 567 & \\
\hline 08470520 & 05/23/2006 14:30 & Pyrrophyta & dinoflagellate & -- & 255,640 & \\
\hline
\end{tabular}


Appendix 1-2.3.c. Data collected at near-surface depth of the Arroyo Colorado near Rio Hondo, Texas, using algal growth and mortality analyses/zooplankton analysis.

[ind. $/ \mathrm{m}^{3}$, number of individuals per cubic meter; total organisms, micro plus macrozooplankton]

\begin{tabular}{ccrr}
\hline $\begin{array}{c}\text { USGS } \\
\text { station no. }\end{array}$ & \multicolumn{1}{c}{$\begin{array}{c}\text { Date and } \\
\text { time }\end{array}$} & $\begin{array}{c}\text { Macro- } \\
\text { zooplankton } \\
\text { (ind./m }{ }^{3} \text { ) }\end{array}$ & $\begin{array}{r}\text { Total } \\
\text { organisms } \\
\text { (ind./m }{ }^{3}\end{array}$ \\
\hline 08470450 & $02 / 22 / 200617: 30$ & 122,000 & 734,000 \\
08470460 & $02 / 22 / 200616: 30$ & 4,070 & 7,830 \\
08470500 & $02 / 22 / 200615: 30$ & 1,610 & 2,030 \\
08470520 & $02 / 22 / 200613: 30$ & 464 & 618 \\
08470450 & $05 / 24 / 200611: 30$ & 279,000 & 929,000 \\
08470460 & $05 / 24 / 200612: 30$ & 0 & $45,700,000$ \\
08470500 & $05 / 24 / 200613: 30$ & 0 & $15,800,000$ \\
08470520 & $05 / 24 / 200614: 30$ & 446,000 & $1,110,000$ \\
\hline
\end{tabular}


Appendix 1-2.4.a. Data collected at near-surface depth of the Arroyo Colorado near Rio Hondo, Texas, using environmental discretesample analysis, February 2006.

[ft, feet; FNMU, formazin nephelometric units; $\mathrm{mmHg}$, millimeters of mercury; $\mathrm{mg} / \mathrm{L}$, milligrams per liter; --, not sampled; PSU, practical salinity units; $\mu \mathrm{S} / \mathrm{cm}$, microsiemens per centimeter at 25 degrees Celsius; ${ }^{\circ} \mathrm{C}$, degrees Celsius; $\mathrm{E}$, estimated; $\mathrm{CaCO}_{3}$, calcium carbonate; $\mathrm{N}$, nitrogen; $\mathrm{NH}_{4}$, ammonia; $\mathrm{NO}_{3}$, nitrate; $\mathrm{P}$, phosphorus; <, less than; $\mu \mathrm{g} / \mathrm{L}$, micrograms per liter]

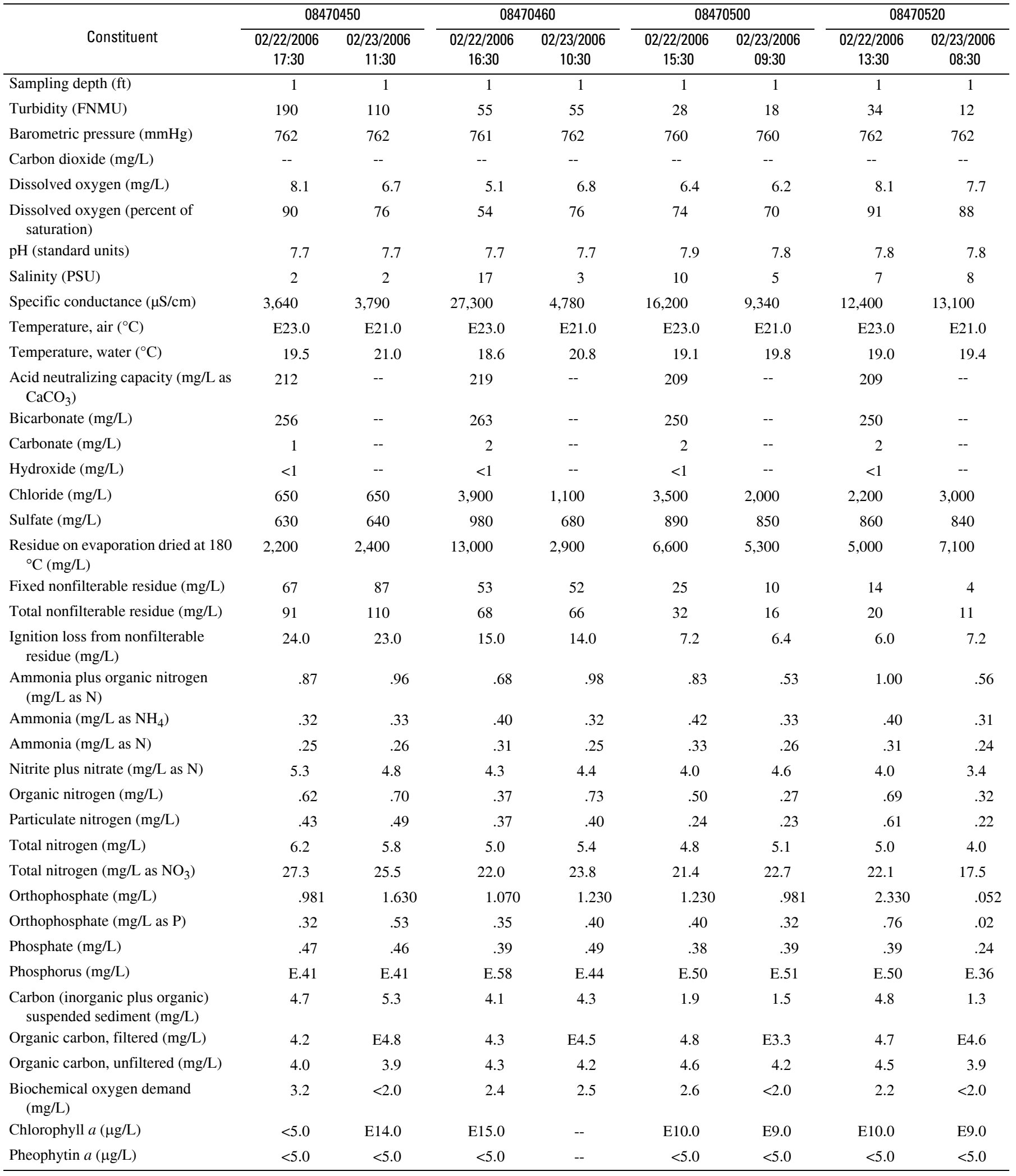


Appendix 1-2.4.b. Data collected at near-surface depth of the Arroyo Colorado near Rio Hondo, Texas, using environmental discretesample analysis, May 2006.

[ft, feet; FNMU, formazin nephelometric units; $\mathrm{mmHg}$, millimeters of mercury; $\mathrm{mg} / \mathrm{L}$, milligrams per liter; --, not sampled; PSU, practical salinity units; $\mu \mathrm{S} / \mathrm{cm}$, microsiemens per centimeter at 25 degrees Celsius; ${ }^{\circ} \mathrm{C}$, degrees Celsius; $\mathrm{CaCO}_{3}$, calcium carbonate; E, estimated; $\mathrm{N}$, nitrogen; $\mathrm{NH}_{4}$, ammonia; $\mathrm{NO}_{3}$, nitrate; $\mathrm{P}$, phosphorus; $\mu \mathrm{g} / \mathrm{L}$, micrograms per liter; $<$, less than]

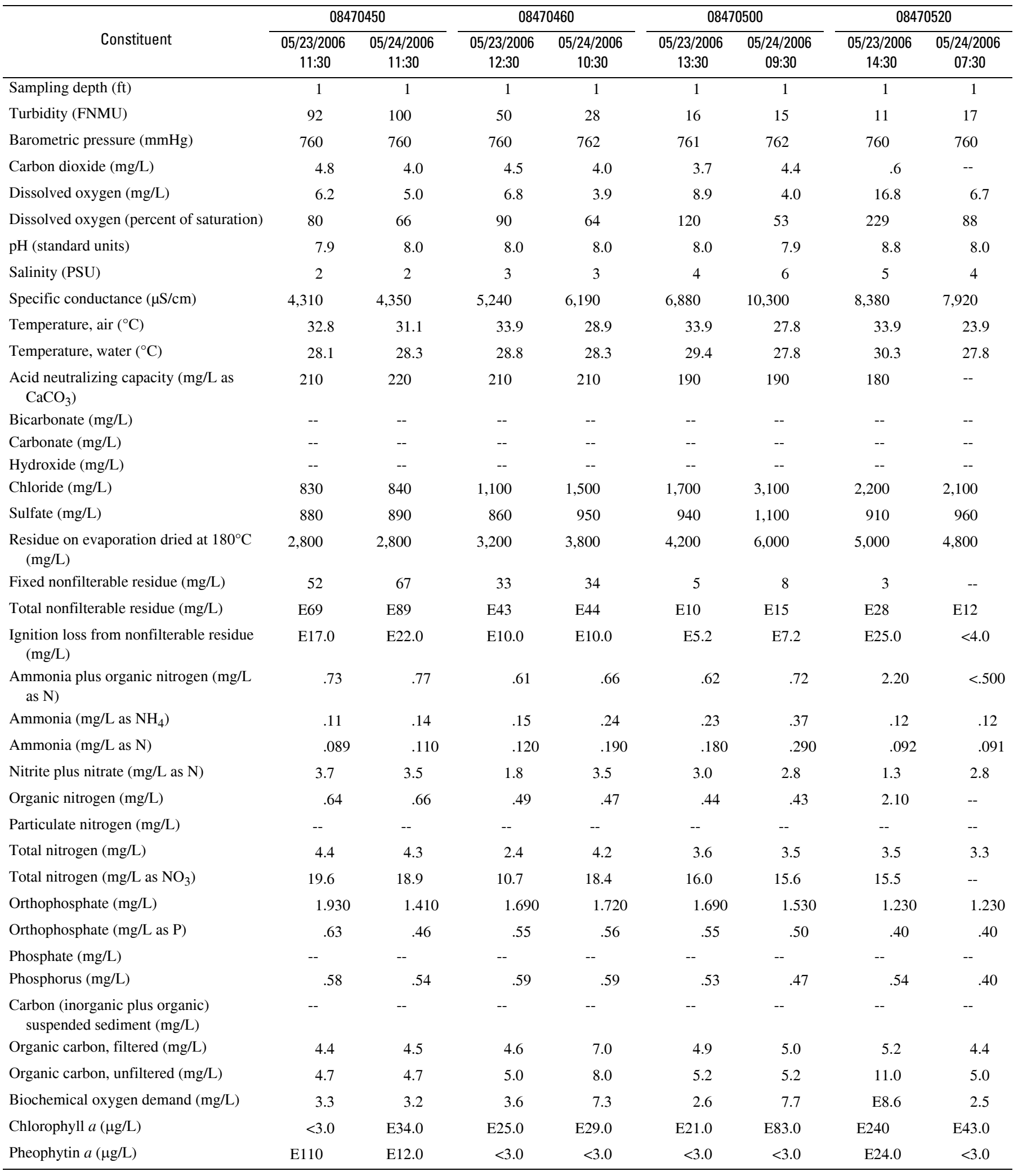


Appendix 1-3. Data collected for quality assurance/quality control from the Arroyo Colorado near Rio Hondo, Texas.

[mg/L, milligrams per liter; $\mathrm{CaCO}_{3}$, calcium carbonate; --, not sampled; <, less than; ${ }^{\circ} \mathrm{C}$, degrees Celsius; $\mathrm{N}$, nitrogen; $\mathrm{NH}_{4}$, ammonia; $\mathrm{NO}_{3}$, nitrate; $\mathrm{P}$, phosphorus; $\mu \mathrm{g} / \mathrm{L}$, micrograms per liter; E, estimated]

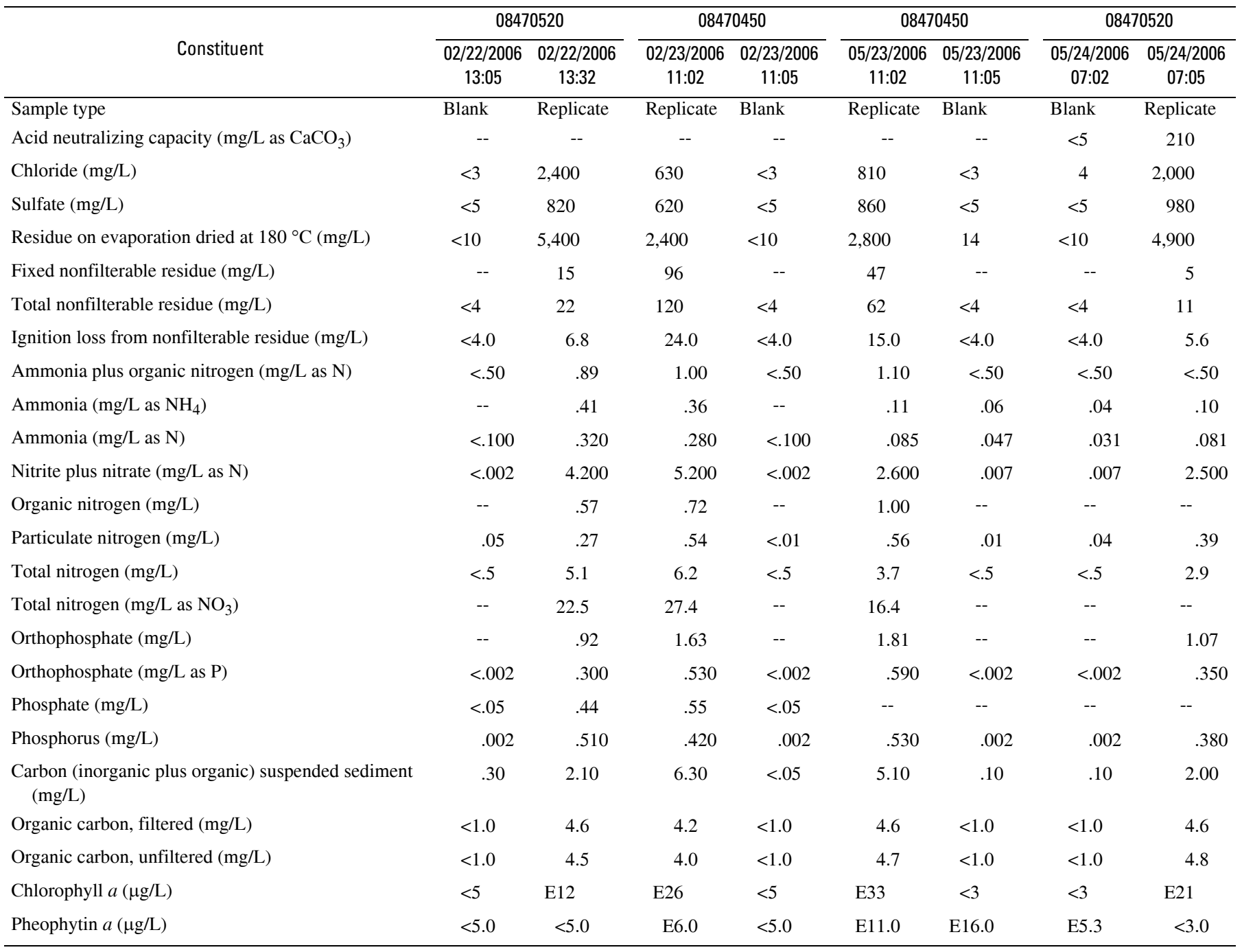


Appendix 2-Vertical Profile Data Collected From the Arroyo Colorado Near Rio Hondo, Texas 
Blank Page 
Appendix 2-1. Water-quality monitor vertical profile data collected from the Arroyo Colorado near Rio Hondo, Texas.

[ft, feet; ${ }^{\circ} \mathrm{C}$, degrees Celsius; $\mu \mathrm{S} / \mathrm{cm}$, microsiemens per centimeter at 25 degrees Celsius; $\mathrm{mg} / \mathrm{L}$, milligrams per liter; PSU, practical salinity units; FNMU, formazin nephelometric units; E, estimated]

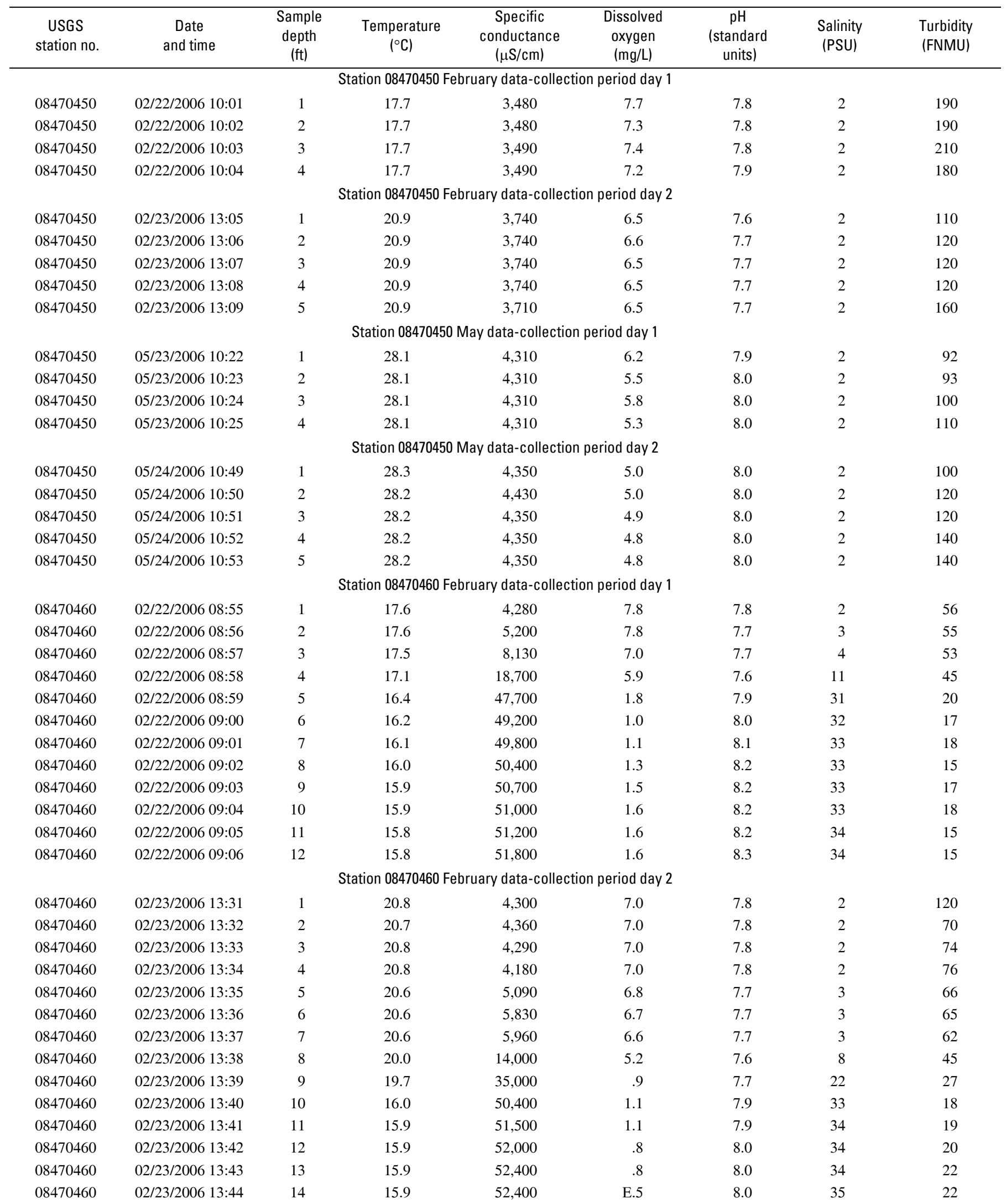


Appendix 2-1. Water-quality monitor vertical profile data collected from the Arroyo Colorado near Rio Hondo, Texas-Continued.

\begin{tabular}{|c|c|c|c|c|c|c|c|c|}
\hline $\begin{array}{c}\text { USGS } \\
\text { station no. }\end{array}$ & $\begin{array}{c}\text { Date } \\
\text { and time }\end{array}$ & $\begin{array}{l}\text { Sample } \\
\text { depth } \\
\text { (ft) }\end{array}$ & $\begin{array}{c}\text { Temperature } \\
\left({ }^{\circ} \mathrm{C}\right)\end{array}$ & $\begin{array}{c}\text { Specific } \\
\text { conductance } \\
(\mu \mathrm{S} / \mathrm{cm})\end{array}$ & $\begin{array}{l}\text { Dissolved } \\
\text { oxygen } \\
\text { (mg/L) }\end{array}$ & $\begin{array}{c}\mathrm{pH} \\
\text { (standard } \\
\text { units) }\end{array}$ & $\begin{array}{l}\text { Salinity } \\
\text { (PSU) }\end{array}$ & $\begin{array}{l}\text { Turbidity } \\
\text { (FNMU) }\end{array}$ \\
\hline \multicolumn{9}{|c|}{ Station 08470460 May data-collection period day 1} \\
\hline 08470460 & 05/23/2006 09:57 & 1 & 27.8 & 4,980 & 5.6 & 8.0 & 3 & 36 \\
\hline 08470460 & 05/23/2006 09:58 & 2 & 27.8 & 5,560 & 5.1 & 7.9 & 3 & 50 \\
\hline 08470460 & 05/23/2006 09:59 & 3 & 27.8 & 6,470 & 4.2 & 7.9 & 4 & 40 \\
\hline 08470460 & 05/23/2006 10:00 & 4 & 27.8 & 9,620 & 4.1 & 7.9 & 5 & 43 \\
\hline 08470460 & 05/23/2006 10:01 & 5 & 27.6 & 17,400 & 1.2 & 7.7 & 10 & 18 \\
\hline 08470460 & 05/23/2006 10:02 & 6 & 27.6 & 18,800 & E.5 & 7.7 & 11 & 20 \\
\hline 08470460 & 05/23/2006 10:03 & 7 & 27.3 & 23,700 & E. 2 & 7.6 & 14 & 34 \\
\hline 08470460 & 05/23/2006 10:04 & 8 & 27.2 & 26,800 & E.1 & 7.8 & 16 & 28 \\
\hline 08470460 & 05/23/2006 10:05 & 9 & 27.0 & 29,700 & E.1 & 8.4 & 18 & 57 \\
\hline 08470460 & 05/23/2006 10:06 & 10 & 26.9 & 32,800 & E.1 & 8.6 & 21 & 38 \\
\hline 08470460 & 05/23/2006 10:07 & 11 & 26.7 & 34,800 & E.1 & 8.6 & 22 & 33 \\
\hline 08470460 & 05/23/2006 10:08 & 12 & 26.6 & 36,300 & E.1 & 8.5 & 23 & 27 \\
\hline \multicolumn{9}{|c|}{ Station 08470460 May data-collection period day 2} \\
\hline 08470460 & 05/24/2006 10:07 & 1 & 28.3 & 6,190 & 3.9 & 8.0 & 3 & 28 \\
\hline 08470460 & 05/24/2006 10:08 & 2 & 28.3 & 6,330 & 4.6 & 8.0 & 3 & 48 \\
\hline 08470460 & 05/24/2006 10:09 & 3 & 28.0 & 9,620 & 3.0 & 7.9 & 5 & 31 \\
\hline 08470460 & 05/24/2006 10:10 & 4 & 27.9 & 16,200 & 1.9 & 7.8 & 9 & 25 \\
\hline 08470460 & 05/24/2006 10:11 & 5 & 27.8 & 18,400 & .9 & 7.7 & 11 & 29 \\
\hline 08470460 & 05/24/2006 10:12 & 6 & 27.7 & 20,300 & E.5 & 7.7 & 12 & 35 \\
\hline 08470460 & 05/24/2006 10:13 & 7 & 27.6 & 22,900 & E.1 & 7.7 & 14 & 48 \\
\hline 08470460 & 05/24/2006 10:14 & 8 & 27.4 & 26,300 & E.1 & 7.8 & 16 & 57 \\
\hline 08470460 & 05/24/2006 10:15 & 9 & 27.3 & 28,000 & E.1 & 8.1 & 17 & 57 \\
\hline 08470460 & 05/24/2006 10:16 & 10 & 27.2 & 29,500 & E.1 & 8.4 & 18 & 65 \\
\hline 08470460 & 05/24/2006 10:17 & 11 & 27.0 & 31,700 & E.1 & 8.7 & 20 & 59 \\
\hline 08470460 & 05/24/2006 10:18 & 12 & 26.8 & 33,900 & E.1 & 8.7 & 21 & 48 \\
\hline 08470460 & 05/24/2006 10:19 & 13 & 26.7 & 35,300 & E.1 & 8.7 & 22 & 36 \\
\hline 08470460 & 05/24/2006 10:20 & 14 & 26.6 & 37,100 & E.1 & 8.6 & 23 & 29 \\
\hline \multicolumn{9}{|c|}{ Station 08470500 February data-collection period day 1} \\
\hline 08470500 & $02 / 22 / 200610: 55$ & 1 & 17.4 & 5,310 & 8.5 & 7.7 & 3 & 28 \\
\hline 08470500 & 02/22/2006 10:56 & 2 & 17.4 & 5,270 & 8.2 & 7.7 & 3 & 28 \\
\hline 08470500 & 02/22/2006 10:57 & 3 & 17.3 & 5,530 & 8.0 & 7.7 & 3 & 28 \\
\hline 08470500 & 02/22/2006 10:58 & 4 & 17.3 & 5,930 & 6.9 & 7.5 & 3 & 21 \\
\hline 08470500 & 02/22/2006 10:59 & 5 & 16.4 & 48,600 & 1.9 & 7.8 & 32 & 13 \\
\hline 08470500 & 02/22/2006 11:00 & 6 & 16.4 & 48,700 & 1.1 & 7.9 & 32 & 11 \\
\hline 08470500 & 02/22/2006 11:01 & 7 & 15.9 & 50,300 & 1.6 & 8.0 & 33 & 9.8 \\
\hline 08470500 & 02/22/2006 11:02 & 8 & 16.0 & 51,400 & 1.6 & 8.1 & 34 & 12 \\
\hline 08470500 & 02/22/2006 11:03 & 9 & 16.0 & 53,200 & 1.5 & 8.1 & 35 & 7.7 \\
\hline 08470500 & 02/22/2006 11:04 & 10 & 16.1 & 53,300 & 1.4 & 8.2 & 35 & 8.0 \\
\hline 08470500 & 02/22/2006 11:05 & 11 & 16.3 & 54,000 & 1.1 & 8.2 & 36 & 6.5 \\
\hline 08470500 & 02/22/2006 11:06 & 12 & 16.4 & 54,000 & 1.1 & 8.2 & 36 & 7.2 \\
\hline 08470500 & 02/22/2006 11:07 & 13 & 16.4 & 54,000 & 1.0 & 8.2 & 36 & 6.8 \\
\hline \multicolumn{9}{|c|}{ Station 08470500 February data-collection period day 2} \\
\hline 08470500 & 02/23/2006 14:05 & 1 & 19.7 & 14,300 & 7.4 & 7.8 & 8 & 18 \\
\hline 08470500 & 02/23/2006 14:06 & 2 & 19.6 & 14,600 & 7.3 & 7.8 & 8 & 18 \\
\hline 08470500 & 02/23/2006 14:07 & 3 & 19.6 & 14,800 & 7.2 & 7.8 & 9 & 19 \\
\hline 08470500 & 02/23/2006 14:08 & 4 & 19.6 & 15,000 & 7.0 & 7.8 & 9 & 21 \\
\hline 08470500 & 02/23/2006 14:09 & 5 & 19.6 & 15,200 & 6.9 & 7.8 & 9 & 12 \\
\hline 08470500 & 02/23/2006 14:10 & 6 & 19.2 & 19,500 & 5.6 & 7.7 & 12 & 20 \\
\hline 08470500 & 02/23/2006 14:11 & 7 & 18.7 & 26,400 & 4.4 & 7.7 & 16 & 18 \\
\hline 08470500 & 02/23/2006 14:12 & 8 & 17.8 & 38,100 & 1.7 & 7.7 & 24 & 16 \\
\hline 08470500 & 02/23/2006 14:13 & 9 & 16.9 & 48,000 & .8 & 7.8 & 31 & 8.4 \\
\hline 08470500 & 02/23/2006 14:14 & 10 & 16.5 & 50,300 & .9 & 7.8 & 33 & 13 \\
\hline
\end{tabular}


Appendix 2-1. Water-quality monitor vertical profile data collected from the Arroyo Colorado near Rio Hondo, Texas-Continued.

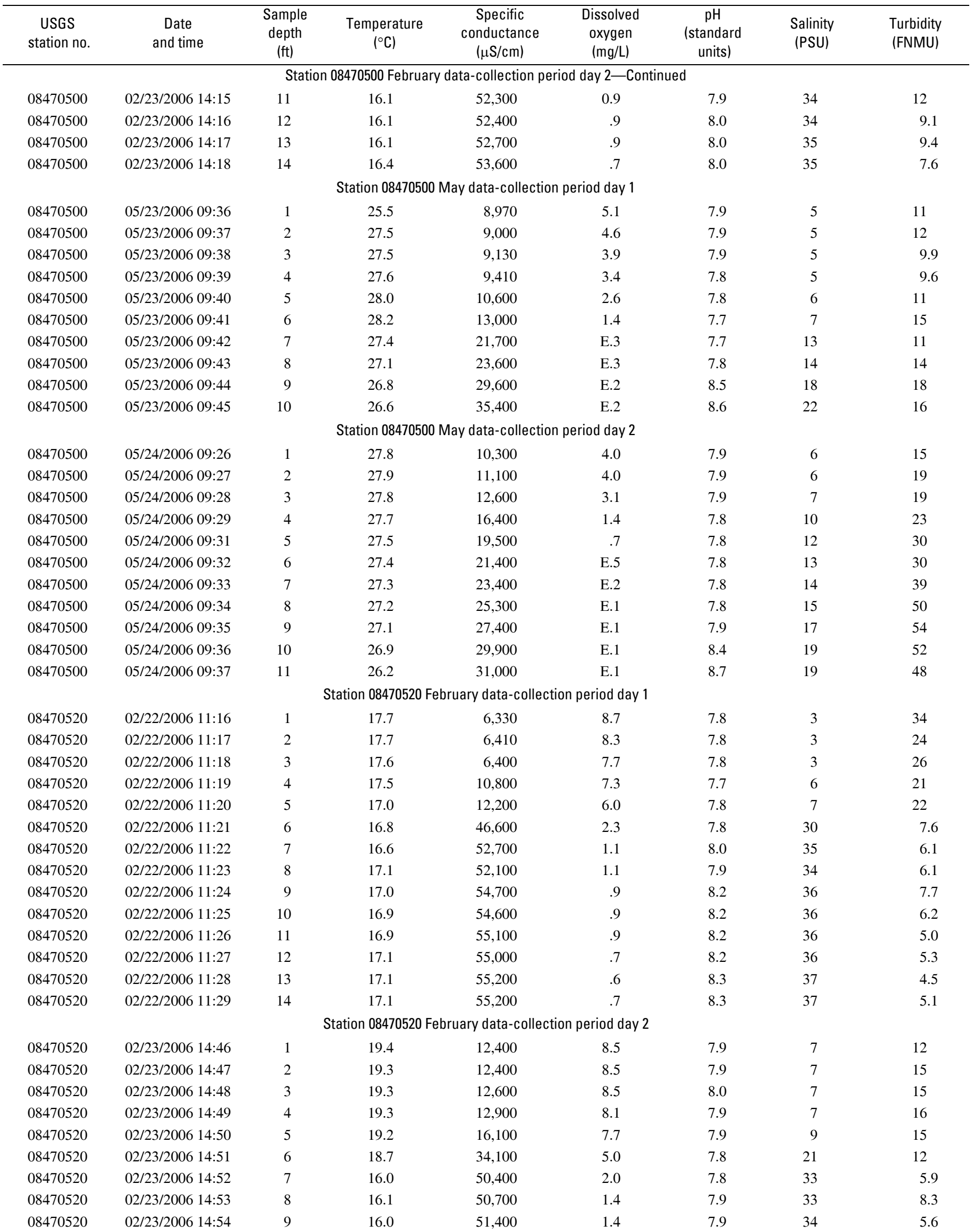


44 Water-Quality and Ancillary Data Collected From the Arroyo Colorado Near Rio Hondo, Texas, 2006

Appendix 2-1. Water-quality monitor vertical profile data collected from the Arroyo Colorado near Rio Hondo, Texas-Continued.

\begin{tabular}{|c|c|c|c|c|c|c|c|c|}
\hline $\begin{array}{c}\text { USGS } \\
\text { station no. }\end{array}$ & $\begin{array}{c}\text { Date } \\
\text { and time }\end{array}$ & $\begin{array}{l}\text { Sample } \\
\text { depth } \\
\text { (ft) }\end{array}$ & $\begin{array}{c}\text { Temperature } \\
\left({ }^{\circ} \mathrm{C}\right)\end{array}$ & $\begin{array}{c}\text { Specific } \\
\text { conductance } \\
(\mu \mathrm{S} / \mathrm{cm})\end{array}$ & $\begin{array}{c}\text { Dissolved } \\
\text { oxygen } \\
\text { (mg/L) }\end{array}$ & $\begin{array}{c}\mathrm{pH} \\
\text { (standard } \\
\text { units) }\end{array}$ & $\begin{array}{l}\text { Salinity } \\
\text { (PSU) }\end{array}$ & $\begin{array}{l}\text { Turbidity } \\
\text { (FNMU) }\end{array}$ \\
\hline \multicolumn{9}{|c|}{ Station 08470520 February data-collection period day 2 -Continued } \\
\hline 08470520 & 02/23/2006 14:56 & 11 & 16.4 & 53,200 & 1.0 & 8.0 & 35 & 7.5 \\
\hline 08470520 & 02/23/2006 14:57 & 12 & 16.8 & 54,400 & E.5 & 8.1 & 36 & 6.6 \\
\hline 08470520 & 02/23/2006 14:58 & 13 & 16.9 & 54,600 & E.4 & 8.1 & 36 & 6.4 \\
\hline 08470520 & 02/23/2006 15:00 & 15 & 17.0 & 54,800 & E.4 & 8.1 & 36 & 6.4 \\
\hline \multicolumn{9}{|c|}{ Station 08470520 May data-collection period day 1} \\
\hline 08470520 & 05/23/2006 09:10 & 1 & 27.5 & 8,040 & 7.5 & 7.9 & 4 & 24 \\
\hline 08470520 & 05/23/2006 09:11 & 2 & 27.5 & 8,510 & 6.6 & 7.8 & 5 & 18 \\
\hline 08470520 & 05/23/2006 09:12 & 3 & 27.7 & 8,430 & 5.3 & 7.8 & 5 & 17 \\
\hline 08470520 & 05/23/2006 09:13 & 4 & 27.8 & 10,500 & 3.2 & 7.5 & 6 & 14 \\
\hline 08470520 & 05/23/2006 09:17 & 8 & 26.7 & 29,200 & E. 2 & 8.5 & 18 & 14 \\
\hline 08470520 & 05/23/2006 09:18 & 9 & 26.7 & 31,500 & E. 2 & 8.6 & 20 & 13 \\
\hline 08470520 & 05/23/2006 09:19 & 10 & 26.4 & 35,500 & E. 2 & 8.7 & 22 & 11 \\
\hline 08470520 & 05/23/2006 09:20 & 11 & 25.9 & 43,100 & E. 2 & 9.0 & 28 & 16 \\
\hline 08470520 & 05/23/2006 09:21 & 12 & 25.9 & 43,300 & E. 2 & 9.1 & 28 & 15 \\
\hline 08470520 & 05/23/2006 09:22 & 13 & 25.7 & 43,900 & E. 2 & 9.1 & 28 & 16 \\
\hline 08470520 & 05/23/2006 09:23 & 14 & 25.7 & 44,000 & E. 2 & 9.1 & 28 & 14 \\
\hline 08470520 & 05/23/2006 09:24 & 15 & 25.7 & 44,100 & E.1 & 9.1 & 28 & 16 \\
\hline \multicolumn{9}{|c|}{ Station 08470520 May data-collection period day 2} \\
\hline 08470520 & 05/24/2006 07:40 & 1 & 27.8 & 7,920 & 6.7 & 8.0 & 4 & 17 \\
\hline 08470520 & 05/24/2006 07:41 & 2 & 28.1 & 8,240 & 6.2 & 8.0 & 5 & 15 \\
\hline 08470520 & 05/24/2006 07:49 & 10 & 25.7 & 44,500 & E.1 & 9.4 & 29 & 16 \\
\hline 08470520 & 05/24/2006 07:50 & 11 & 25.5 & 44,900 & E.1 & 9.4 & 29 & 15 \\
\hline 08470520 & 05/24/2006 07:51 & 12 & 25.5 & 45,000 & E.1 & 9.5 & 29 & 18 \\
\hline 08470520 & 05/24/2006 07:52 & 13 & 25.5 & 45,000 & E.1 & 9.5 & 29 & 16 \\
\hline 08470520 & 05/24/2006 07:53 & 14 & 25.5 & 45,000 & E.1 & 9.5 & 29 & 17 \\
\hline 08470520 & 05/24/2006 07:54 & 15 & 25.5 & 45,100 & E.1 & 9.5 & 29 & 16 \\
\hline
\end{tabular}


Appendix 2-2. PAR sensor vertical profile data collected from the Arroyo Colorado near Rio Hondo, Texas.

[PAR, photosynthetically active radiation; $\mathrm{m}$, meters; $\mu \mathrm{E} / \mathrm{m}^{2} / \mathrm{s}$, microEinsteins per square meter per second]

\begin{tabular}{|c|c|c|c|c|c|}
\hline $\begin{array}{c}\text { USGS } \\
\text { station no. }\end{array}$ & $\begin{array}{l}\text { Date and } \\
\text { time }\end{array}$ & $\begin{array}{c}\text { Sample } \\
\text { depth } \\
\text { (m) }\end{array}$ & Remark & $\begin{array}{c}\text { Downwelling light } \\
\left(\mu \mathrm{E} / \mathrm{m}^{2} / \mathrm{sec}\right)\end{array}$ & $\begin{array}{c}\text { Upwelling light } \\
\left(\mu \mathrm{E} / \mathrm{m}^{2} / \mathrm{sec}\right)\end{array}$ \\
\hline \multicolumn{6}{|c|}{ Station 08470450 February data-collection period } \\
\hline 08470450 & $02 / 22 / 200614: 20$ & 0 & Air & $1,774.00$ & 152.00 \\
\hline 08470450 & $02 / 22 / 200614: 21$ & 0 & Surface & 644.00 & 29.00 \\
\hline 08470450 & $02 / 22 / 200614: 22$ & 1 & Water & 11.00 & .60 \\
\hline 08470450 & $02 / 22 / 200614: 23$ & 2 & Water & .01 & .00 \\
\hline \multicolumn{6}{|c|}{ Station 08470450 May data-collection period } \\
\hline 08470450 & 05/23/2006 14:20 & 0 & Air & $2,430.00$ & 109.10 \\
\hline 08470450 & 05/23/2006 14:21 & 0 & Surface & $1,274.00$ & 38.01 \\
\hline 08470450 & 05/23/2006 14:22 & 1 & Water & 2.30 & .20 \\
\hline 08470450 & 05/23/2006 14:23 & 2 & Water & .00 & .00 \\
\hline \multicolumn{6}{|c|}{ Station 08470460 February data-collection period } \\
\hline 08470460 & 02/22/2006 10:22 & 0 & Air & 207.00 & 22.00 \\
\hline 08470460 & 02/22/2006 10:23 & 0 & Surface & 82.00 & 12.00 \\
\hline 08470460 & 02/22/2006 10:24 & 1 & Water & 25.00 & 3.00 \\
\hline 08470460 & 02/22/2006 10:25 & 2 & Water & 3.00 & .03 \\
\hline 08470460 & 02/22/2006 10:26 & 3 & Water & 1.00 & .20 \\
\hline 08470460 & 02/22/2006 10:27 & 4 & Water & .30 & .04 \\
\hline 08470460 & $02 / 22 / 200614: 32$ & 0 & Air & 837.00 & 126.00 \\
\hline 08470460 & $02 / 22 / 200614: 33$ & 0 & Surface & 399.00 & 19.00 \\
\hline 08470460 & 02/22/2006 14:34 & 1 & Water & 41.00 & 4.00 \\
\hline 08470460 & 02/22/2006 14:35 & 2 & Water & 4.70 & .60 \\
\hline 08470460 & 02/22/2006 14:36 & 3 & Water & .80 & .10 \\
\hline 08470460 & 02/22/2006 14:37 & 4 & Water & .40 & .06 \\
\hline 08470460 & 02/22/2006 16:08 & 0 & Air & 940.00 & 182.00 \\
\hline 08470460 & 02/22/2006 16:09 & 0 & Surface & 544.00 & 51.00 \\
\hline 08470460 & 02/22/2006 16:10 & 1 & Water & 83.00 & 7.00 \\
\hline 08470460 & 02/22/2006 16:11 & 2 & Water & 13.00 & 1.40 \\
\hline 08470460 & 02/22/2006 16:12 & 3 & Water & 2.80 & .30 \\
\hline 08470460 & 02/22/2006 16:13 & 4 & Water & .40 & .06 \\
\hline \multicolumn{6}{|c|}{ Station 08470460 May data-collection period } \\
\hline 08470460 & 05/23/2006 10:22 & 0 & Air & $1,820.00$ & 137.00 \\
\hline 08470460 & 05/23/2006 10:23 & 0 & Surface & 275.00 & 48.00 \\
\hline 08470460 & 05/23/2006 10:24 & 1 & Water & 7.20 & 1.60 \\
\hline 08470460 & 05/23/2006 10:25 & 2 & Water & .43 & .09 \\
\hline 08470460 & 05/23/2006 10:26 & 3 & Water & .02 & .00 \\
\hline 08470460 & 05/23/2006 10:27 & 4 & Water & .00 & .00 \\
\hline \multicolumn{6}{|c|}{ Station 08470500 February data-collection period } \\
\hline 08470500 & 02/22/2006 10:48 & 0 & Air & 856.00 & 85.00 \\
\hline 08470500 & 02/22/2006 10:49 & 0 & Surface & 425.00 & 43.00 \\
\hline 08470500 & $02 / 22 / 200610: 50$ & 1 & Water & 114.00 & 12.00 \\
\hline 08470500 & $02 / 22 / 200610: 51$ & 2 & Water & 13.00 & 1.10 \\
\hline 08470500 & $02 / 22 / 200610: 52$ & 3 & Water & 3.60 & .40 \\
\hline 08470500 & $02 / 22 / 200610: 53$ & 4 & Water & 1.40 & .10 \\
\hline
\end{tabular}




\section{Water-Quality and Ancillary Data Collected From the Arroyo Colorado Near Rio Hondo, Texas, 2006}

Appendix 2-2. PAR sensor vertical profile data collected from the Arroyo Colorado near Rio Hondo, Texas-Continued.

\begin{tabular}{|c|c|c|c|c|c|}
\hline $\begin{array}{c}\text { USGS } \\
\text { station no. }\end{array}$ & $\begin{array}{l}\text { Date and } \\
\text { time }\end{array}$ & $\begin{array}{c}\text { Sample } \\
\text { depth } \\
\text { (m) }\end{array}$ & Remark & $\begin{array}{l}\text { Downwelling light } \\
\left(\mu \mathrm{E} / \mathrm{m}^{2} / \mathrm{sec}\right)\end{array}$ & $\begin{array}{l}\text { Upwelling light } \\
\left(\mu \mathrm{E} / \mathrm{m}^{2} / \mathrm{sec}\right)\end{array}$ \\
\hline \multicolumn{6}{|c|}{ Station 08470500 February data-collection period - Continued } \\
\hline 08470500 & 02/22/2006 14:02 & 0 & Air & $1,127.00$ & 142.00 \\
\hline 08470500 & 02/22/2006 14:03 & 0 & Surface & 544.00 & 53.00 \\
\hline 08470500 & 02/22/2006 14:04 & 1 & Water & 132.00 & 14.00 \\
\hline 08470500 & 02/22/2006 14:05 & 2 & Water & 8.00 & .90 \\
\hline 08470500 & 02/22/2006 14:06 & 3 & Water & 2.20 & .20 \\
\hline 08470500 & 02/22/2006 14:07 & 4 & Water & .70 & .09 \\
\hline 08470500 & $02 / 22 / 200616: 28$ & 0 & Air & 814.00 & 139.00 \\
\hline 08470500 & 02/22/2006 16:29 & 0 & Surface & 441.00 & 47.00 \\
\hline 08470500 & 02/22/2006 16:30 & 1 & Water & 115.00 & 9.00 \\
\hline 08470500 & 02/22/2006 16:31 & 2 & Water & 16.00 & 1.00 \\
\hline 08470500 & 02/22/2006 16:32 & 3 & Water & 3.80 & .30 \\
\hline \multicolumn{6}{|c|}{ Station 08470500 May data-collection period } \\
\hline 08470500 & 05/23/2006 10:48 & 0 & Air & 477.00 & 69.00 \\
\hline 08470500 & 05/23/2006 10:49 & 0 & Surface & 276.00 & 53.00 \\
\hline 08470500 & 05/23/2006 10:50 & 1 & Water & 36.00 & 8.90 \\
\hline 08470500 & 05/23/2006 10:51 & 2 & Water & 15.00 & 2.30 \\
\hline 08470500 & 05/23/2006 10:52 & 3 & Water & 2.80 & .35 \\
\hline 08470500 & 05/23/2006 10:53 & 4 & Water & .58 & .05 \\
\hline \multicolumn{6}{|c|}{ Station 08470520 February data-collection period } \\
\hline 08470520 & 02/22/2006 13:30 & 0 & Air & $1,633.00$ & 174.00 \\
\hline 08470520 & 02/22/2006 13:31 & 0 & Surface & 938.00 & 91.00 \\
\hline 08470520 & 02/22/2006 13:32 & 1 & Water & 217.00 & 26.00 \\
\hline 08470520 & $02 / 22 / 200613: 33$ & 2 & Water & 23.00 & 2.80 \\
\hline 08470520 & $02 / 22 / 200613: 34$ & 3 & Water & 4.10 & .40 \\
\hline 08470520 & 02/22/2006 13:35 & 4 & Water & 1.40 & .10 \\
\hline \multicolumn{6}{|c|}{ Station 08470520 May data-collection period } \\
\hline 08470520 & 05/23/2006 13:30 & 0 & Air & $1,737.00$ & 26.07 \\
\hline 08470520 & 05/23/2006 13:31 & 0 & Surface & 148.10 & 3.20 \\
\hline 08470520 & 05/23/2006 13:32 & 1 & Water & 3.46 & .51 \\
\hline 08470520 & 05/23/2006 13:33 & 2 & Water & .36 & .04 \\
\hline 08470520 & 05/23/2006 13:34 & 3 & Water & .08 & .01 \\
\hline
\end{tabular}

Prepared by the USGS Texas Water Science Center:

8027 Exchange Drive

Austin, TX 78754-4733

Information regarding water resources in Texas is available at http: //tx.usgs.gov/ 